Aus der Abteilung Mund- ,Kiefer- und Gesichtschirurgie

(Prof. Dr. med. Dr. med. dent. H. Schliephake)

im Zentrum Zahn- , Mund- und Kieferheilkunde

der Medizinischen Fakultät der Universität Göttingen

\title{
Darstellbarkeit von Knochenstrukturen und enossalen Implantaten mit der \\ Flächendetektor-Volumencomputertomographie und mit konventionellen radiologischen Verfahren
}

Ein Methodenvergleich

\author{
INAUGURAL-DISSERTATION \\ zur Erlangung des Doktorgrades \\ der Medizinischen Fakultät \\ der Georg-August-Universität zu Göttingen
}

vorgelegt von

Lena Jarnot, geb. Nielebock

aus

Göttingen

Göttingen 2010 
Dekan: Prof. Dr. med. C. Frömmel

I. Berichterstatterin: PD Dr. med. dent. S. Sennhenn-Kirchner

II. Berichterstatter/in: Prof. Dr. med. Marten-Engelke

III. Berichterstatter/in:

Tag der mündlichen Prüfung: 25.01.2011 


\section{Inhaltsverzeichnis:}

Kapitel

Seite

1.

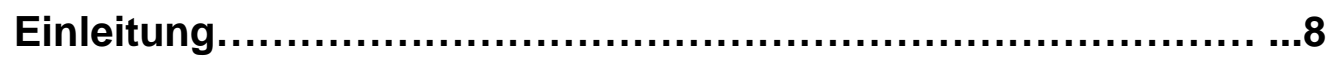

1.1. Die enossale Implantologie.............................................

1.1.1. Enossale Implantate ................................................11

1.1.2. Präoperative Diagnostik und Behandlungsplanung...................13

1.2. Konventionelle Bildgebungsverfahren (Dentalradiologie)................15

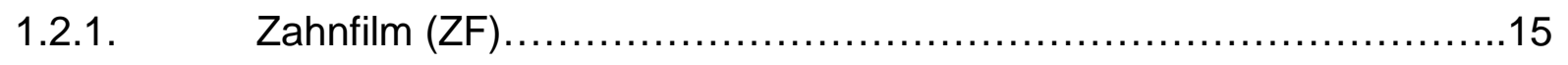

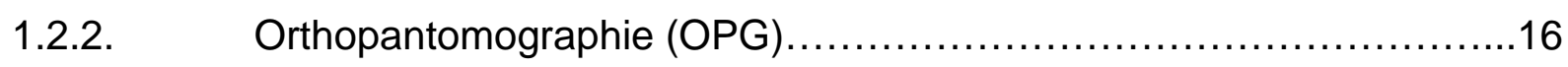

1.3. Dreidimensionale bildgebende Verfahren in der Zahn-, Mund- und

Kieferheilkunde ........................................................ 18

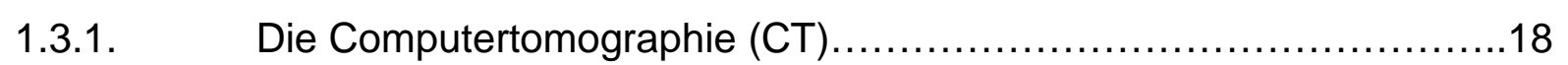

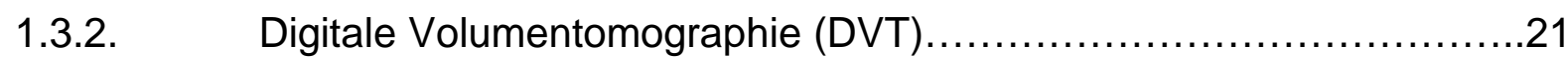

1.3.3. Die flatpanel-Volumencomputertomographie (fpVCT) ..................25

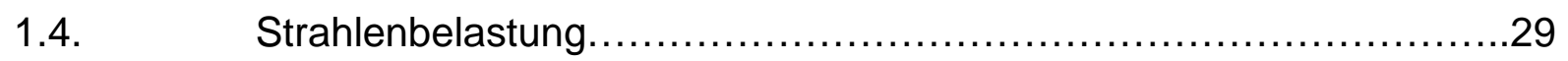

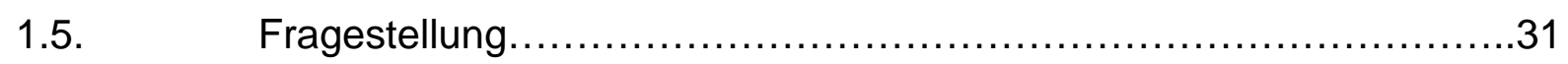

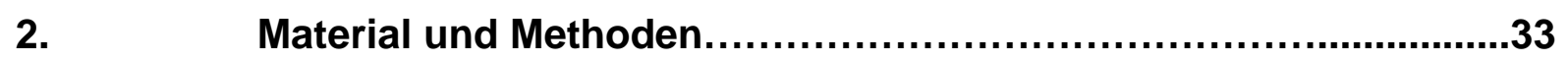

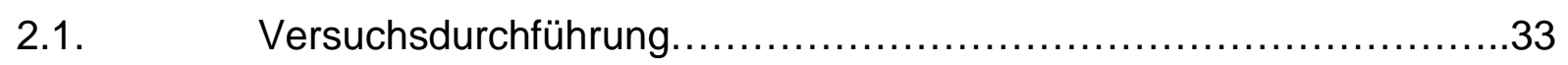

2.1.1. Überblick über die Versuchsdurchführung..............................33

2.1.2. Fließdiagramm der Versuchsdurchführung............................34

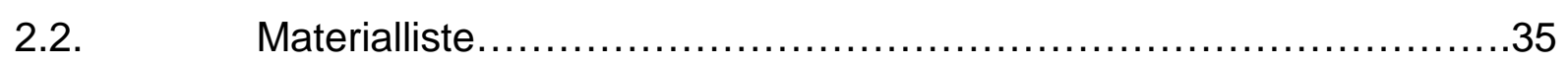

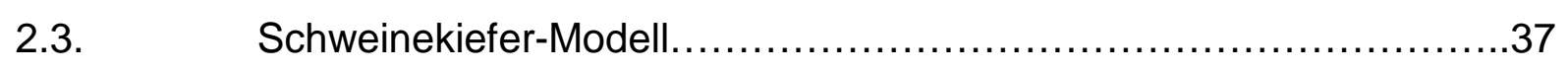

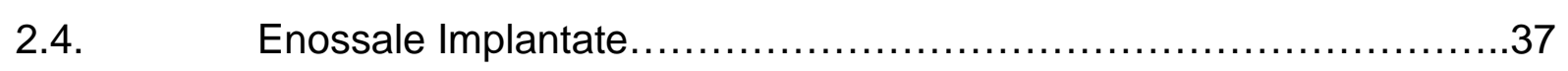

2.4.1. Defekte an Implantaten...............................................

2.5. Präparation des Implantatbettes und Insertion der Implantate............39

2.6. Künstliche Defekte an Alveole und Positionierung von

Fremdkörpern............................................................... 41

2.7. Röntgenologische Verfahren........................................... 44

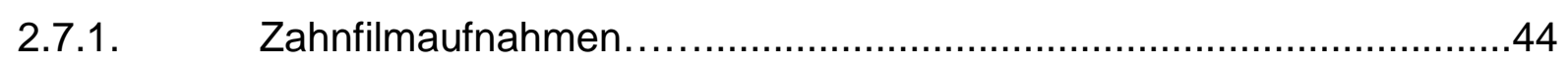

2.7.2. Orthopantomographie (OPAN) ....................................46

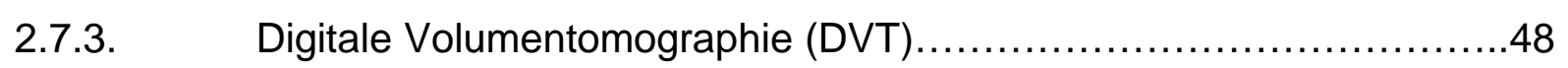




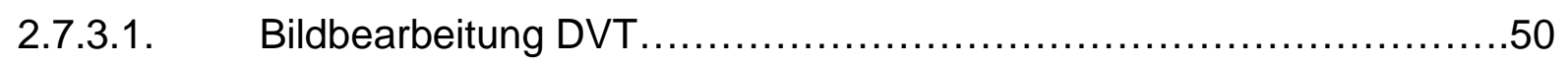

2.7.4. flatpanel-Volumencomputertomographie (fpVCT) ....................50

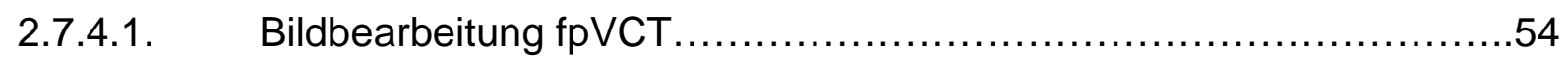

2.8. Geblindete Evaluation der Bilddatensätze durch drei Untersucher......56

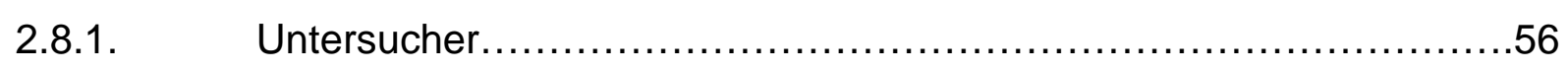

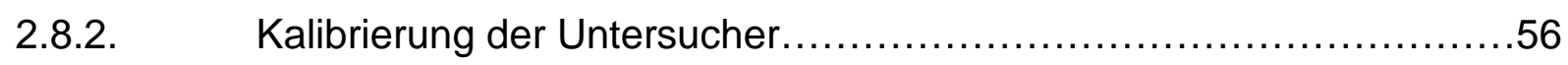

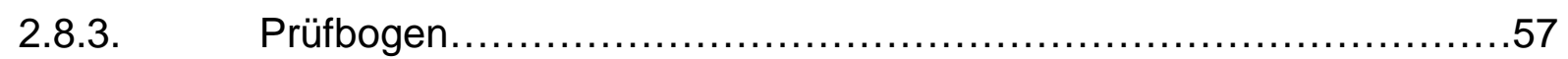

2.8.4. Blindung und Randomisierung der Datensätze .........................59

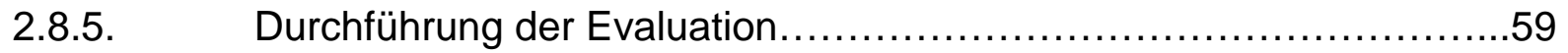

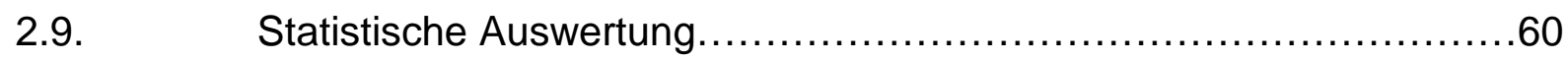

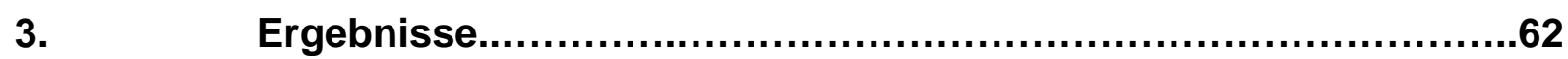

3.1. Bildbeispiele zur Darstellbarkeit von Implantaten und periimplantären Strukturen mit verschiedenen radiologischen Verfahren..................62

3.2. Vergleich der radiologischen Verfahren...............................68

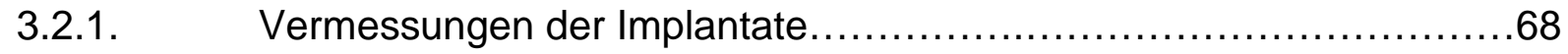

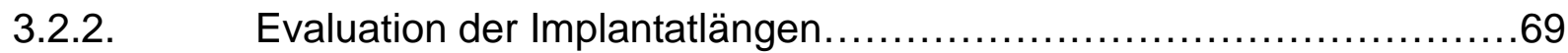

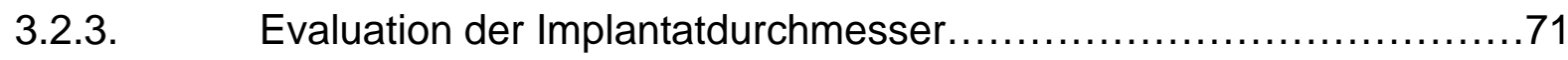

3.2.4. Evaluation der iatrogen applizierten Knochen- bzw. Implantatdefekte

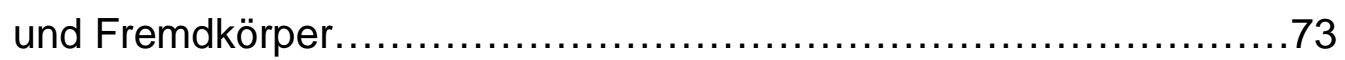

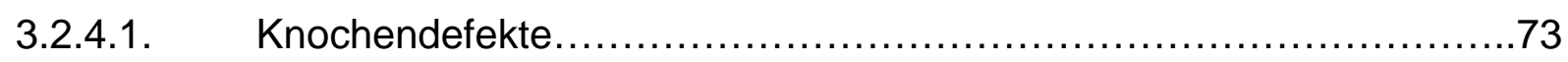

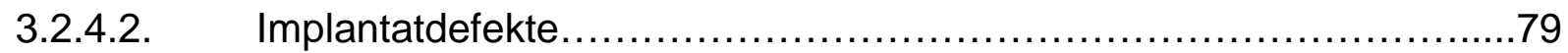

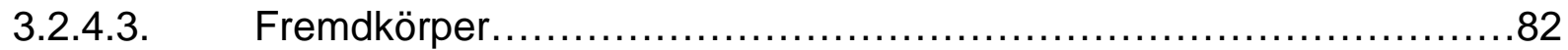

3.2.5. Beeinträchtigung der Bildauswertung durch Streustrahlenartefakte....88

3.3. Zweidimensionale versus dreidimensionale Röntgenverfahren .........88

3.4. Differenzierte Analyse der Fremdkörperdetektion......................91

3.4.1. Fremdkörperaufdeckung: Vergleich der Röntgenmethoden..............92

3.5. Vergleich der Untersucher ............................................. 93

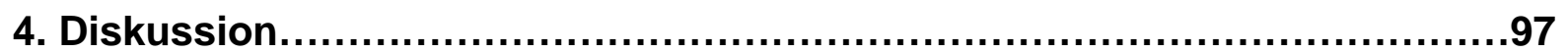

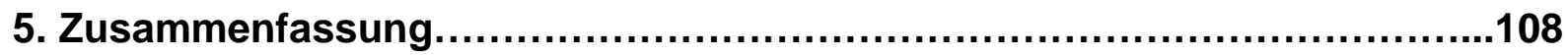

6. Literaturverzeichnis.................................................................110 
Danksagung...

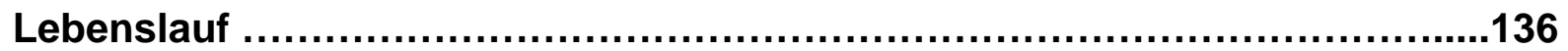




\section{Abkürzungsverzeichnis:}

a-Se $=$ amorphes Selen

$\mathrm{a}-\mathrm{Si}=$ amorphes Silizium

$\beta$-TCP $=$ beta-Tricalciumphosphat

$\mathrm{CBCT}=$ cone-beam Computertomographie

$\mathrm{CCD}=$ Charged Coupled Device

CsI:TI = thalliumdotiertes Cäsiumjodid

CsJ = Cäsiumjodid

$\mathrm{CT}$ = Computertomographie

CTDI = Computertomographie-Dosisindex

CTDIvol = volumenbezogener Computertomographie-Dosisindex

CTDIw = gewichteter Computertomographie-Dosisindex

DICOM = Digital Imaging and Communication in Medicine

$3 \mathrm{D}=$ dreidimensional

$2 \mathrm{D}=$ zweidimensional

$\mathrm{D} / \mathrm{L}=$ Durchmesser/ Länge

DLP $=$ Dosislängenprodukt

$\mathrm{DQE}=$ detective quantum efficiency

$D R G=$ Deutsche Röntgengesellschaft

DVT $=$ Digitale/ $-r$ Volumentomograph/ -ie

Fa. $=$ Firma

$\mathrm{FD}=$ Flachbilddetektor

$\mathrm{FOV}=$ Field of View

fpVCT = flatpanel-Volumencomputertomograph $/$-ie

$\mathrm{GE}=$ General Electric

Gebr. = Gebrüder

$\mathrm{GP}=$ Guttaperchastift

$H E=$ Hounsfield-Einheit

ICRP = international commission on radiological protection

KIS/RIS = Krankenhaus-/ Radiologieinformationssystem

$\mathrm{kV}=$ KiloVolt

$\mathrm{kW}=$ KiloWatt

$\mathrm{Lp} / \mathrm{mm}=$ Linienpaar/ Millimeter 


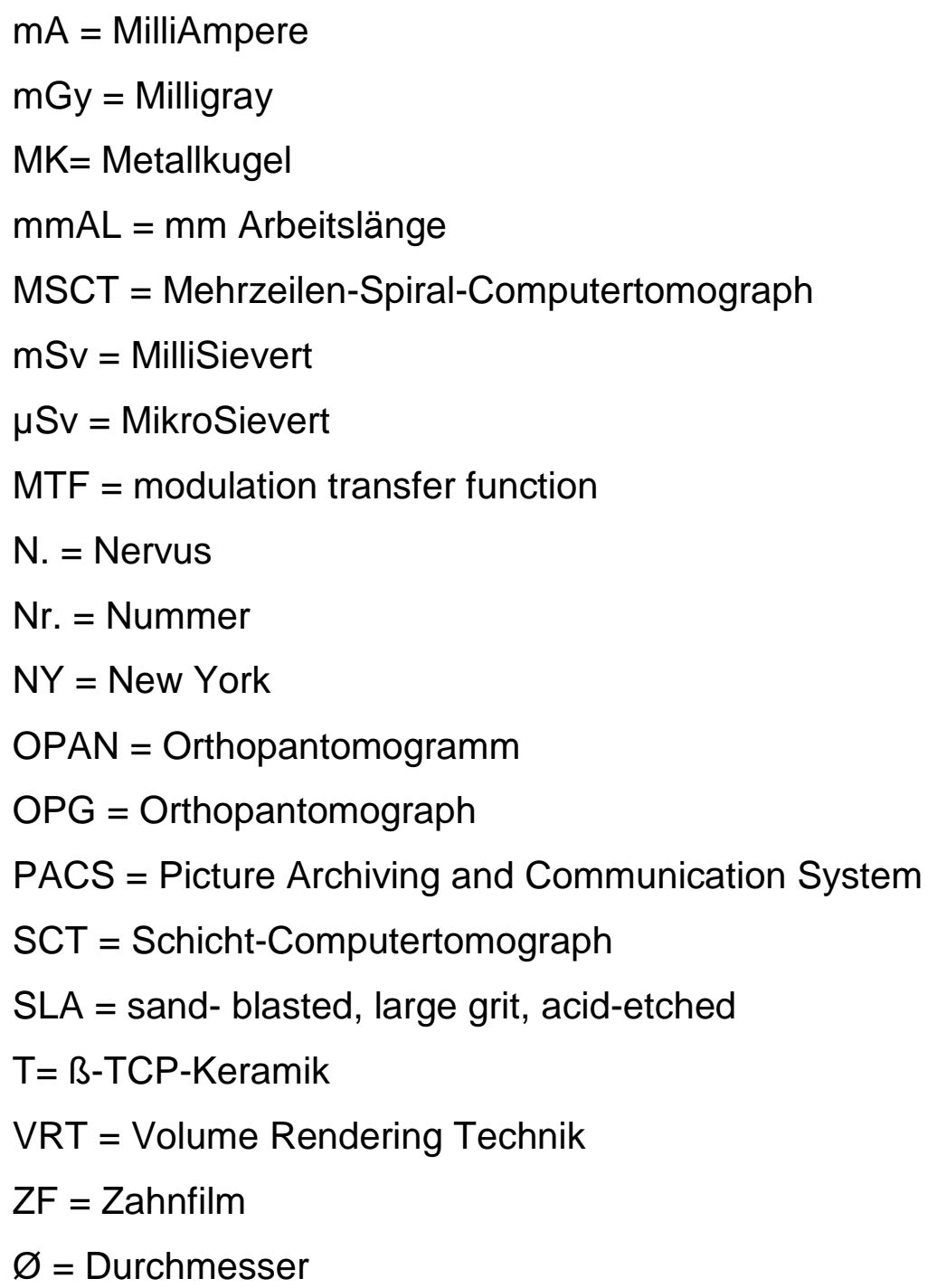




\section{Einleitung}

Mit der seit 1982 wissenschaftlich anerkannten Methode der enossalen Implantologie wurde die Zahn-, Mund- und Kieferheilkunde in den vergangenen 28 Jahren entscheidend bereichert. Während Zahnärzte noch vor etwa 2 Jahrzehnten der zahnärztlichen Implantologie eher skeptisch gegenüber standen, gibt es heute kaum noch praktizierende Oralchirurgen, die sich nicht dem Einsatz enossaler Implantate widmen (Jivraj und Chee 2006). Durch ihre Verwendbarkeit als Träger von Zahnersatz übernehmen Zahnimplantate die Funktion künstlicher Zahnwurzeln und sind somit aus der Zahnmedizin nicht mehr wegzudenken.

Seit 1965 wird der Werkstoff Titan verwendet und prägt die moderne Implantologie. Aufgrund seiner hohen Biokompatibilität und damit guten Verträglichkeit, seiner Stabilität und Korrosionsbeständigkeit stellt er das am meisten verbreitete Material für dentale Implantate dar.

Für ein erfolgreiches Langzeitergebnis einer Implantatinsertion ist sowohl die primäre als auch die sekundäre Stabilität eines Implantates relevant, die miteinander in Zusammenhang stehen (Rodrigo et al. 2010).

Die primäre Stabilität bezieht sich auf die Festigkeit des Implantates nach Insertion in den Knochen, während die sekundäre Stabilität die Festigkeit nach der Einheilungsphase im Knochen bedeutet. Um primäre Stabilität zu erreichen, ist für die Implantatinsertion unter anderem ein ausreichendes Angebot an Knochensubstanz erforderlich (Beer et al. 2003, Molly 2006, Fuh et al. 2010). So sind neben der erfolgreichen Insertion des Implantates in den Kieferkamm auch die präoperative Diagnostik der zu implantierenden Region und die Behandlungsplanung von wesentlicher Bedeutung.

Vor allem die präoperative Beurteilung des vorliegenden Knochenangebotes und der Knochenqualität erlaubt eine Vorhersage über eine gute Osseointegration und damit den Erfolg der Implantatinsertion (Jacobs und van Steenberghe 1998).

Außerdem sind Kenntnisse anatomischer Strukturen notwendig, die bei der Implantation einer möglichen Traumatisierung ausgesetzt sind (BouSerhal et al. 2002, Mraiwa et al. 2003). Die klinische Untersuchung beinhaltet dabei die Beurteilung von morphologischen Besonderheiten des Implantationsgebietes wie die Ermittlung der vorhandenen Qualität und Quantität des Knochens. Weiterhin sind Informationen über vorliegende Pathologien und die Inklination des 
Alveolarfortsatzes notwendig. Bedeutend ist außerdem die Beurteilung der räumlichen Beziehung des Implantationsgebietes zu anatomisch wichtigen Strukturen wie dem Sinus maxillaris, dem Canalis mandibulae, dem N. alveolaris inferior mit dem Foramen mentale (Monsour und Dudhia 2008).

Um mögliche Verletzungen dieser Strukturen im Implantationsgebiet zu vermeiden, bedarf es ausführlicher und genauer Bildgebungsverfahren. Als Goldstandard gilt hierbei der Orthopantomograph, welcher Übersichtsaufnahmen des gesamten Oberund Unterkiefers einschließlich der angrenzenden Kieferhöhlen und -gelenke ermöglicht. Für Routineuntersuchungen im Rahmen einer Implantatplanung im horizontalen Unterkieferast stellt die Panoramaschichtaufnahme ein sicheres Untersuchungsverfahren dar (Vazquez et al. 2007). Da der Orthopantomograph lediglich eine zweidimensionale Übersichtsaufnahme liefert, können relevante anatomische Details von wichtigen Strukturen wie z.B. des N. alveolaris inferior nicht immer ausreichend dargestellt werden. Hier bedarf es häufig weiterführender diagnostischer Verfahren, um eine komplikationslose Implantatinsertion durchführen zu können. Intraorale und extraorale Röntgenaufnahmen kommen zwar mit einer niedrigen Strahlendosis aus, sind jedoch hinsichtlich ihrer Bildinformationen durch ihre Zweidimensionalität begrenzt (Monsour und Dudhia 2008, Patel et al. 2009). Röntgenverfahren mit dreidimensionaler Darstellungsweise, wie z.B. die Computertomographie (CT) oder die digitale Volumentomographie (DVT), haben sich deshalb bewährt und werden empfohlen (Patel et al. 2007). Aufgrund der Dreidimensionalität und der hoch differenzierten Darstellbarkeit können neben Oberund Unterkiefer die Strukturen des Kiefers und kleine verletzungsgefährdete Strukturen im Implantationsgebiet präzise beurteilt werden. Insbesondere wird die 3D-Darstellung auch bei komplexeren Fällen empfohlen, bei denen mehrere Implantate notwendig sind (Garg und Vicari 1995). Dreidimensionale computertomographische Bilddaten ermöglichen eine zuverlässigere Implantatplanung als zweidimensionale Daten (Jacobs et al. 1999). Heute stehen verschiedene computergestützte 3D-Verfahren zur Verfügung. Diese beruhen jedoch zum Teil auf verschiedenartigen Technologien, so dass sie mit unterschiedlichen Vor- und Nachteilen einhergehen, wodurch sie einer entsprechenden Indikationsstellung und weiterer Erforschung bedürfen. Viele Jahre stellten Computertomographen (CT) den Goldstandard dar, wenn präoperativ komplexere Untersuchungen erforderlich waren. Sie lieferten 
dreidimensionale Datensätze mit hoher Detailgenauigkeit (Monsour und Dudhia 2008).

Seither galt die dentale CT als das Standardverfahren zur überlagerungsfreien Darstellung dentaler Strukturen, obwohl ihr Indikationsbereich lange wegen der relativ hohen Strahlenbelastung stark eingeschränkt war (Schmuth et al.1995). Erst seit der Einführung der dosisreduzierten $\mathrm{CT}$, die trotz stark verringerter Strahlenbelastung keine Einbußen in der Abbildungsqualität brachte (Thiel und Hassfeld 2001), kam es zur deutlichen Erweiterung des Indikationsspektrums. Beim Einsatz herkömmlicher handelsüblicher Dental-CT muss jedoch ein Risiko-NutzenAbgleich bezüglich der noch relativ hohen Strahlenbelastung für den Patienten erfolgen und zudem der hohe Kostenaufwand berücksichtigt werden, was eine strenge Indikationsstellung erfordert. Daher hat sich die KegelstrahlVolumentomographie (cone-beam CT) bewährt, die speziell entwickelt wurde, um verzerrungsfreie dreidimensionale Bilddaten des Kiefers, inklusive der Zähne und dem umliegenden Gewebe dosiseffizient akquirieren zu können (Patel 2009). Im Vergleich zum Dental-CT geht sie mit einer geringeren Strahlenbelastung einher (Guerrero et al. 2006) und stellt somit ein vielversprechendes Verfahren zur präoperativen Diagnostik für Zahnimplantate dar (Monsour und Dudhia 2008). Zudem kommt es zu geringerem Auftreten von Metallartefakten, was auch die diagnostische Beurteilung von Strukturen ermöglicht, die in der Nähe von metallischen Restaurationen liegen, während im konventionellen CT Metallartefakte ganze Schichten der Diagnostik entziehen können (Flinzberg et al. 2003, Holberg et al. 2005). DVT gehen allerdings mit einer Erhöhung des Hintergrundrauschens bei gleichzeitiger Kontrastverminderung einher, was die diagnostische Beurteilung feiner dentaler Strukturen in den axialen Schichten und bei den 3D-Darstellungen erschwert (Holberg et al. 2005). Zudem ist keine Weichteildifferenzierung möglich (Holberg et al. 2005, Eggers et al. 2006).

Mit der Entwicklung von Flachbilddetektoren wurde eine neue Technik eingeführt, die weitere Vorteile insbesondere für die CT versprach. Die Entwicklung der Flachdetektor-(FD-)Technologie zielte darauf ab, die Standardradiographie, wie FilmFolien-Kombinationen und Bildverstärker, durch die Bereitstellung höherer Dynamik, Verzerrungsfreiheit und einer gesteigerten Dosiseffizienz zu verbessern. Weitere Vorteile sind zudem die Anfertigung von Serienaufnahmen, eine sofortige Digitalausgabe und eine kompakte Bauweise (Kyriakou et al. 2009). Die Einführung 
der FD-Technologie brachte gegenüber Bildverstärker-basierten Systemen außerdem den entscheidenden Vorteil einer verbesserten Weichteilbildgebung. Somit besitzen die FD-basierten Systeme durch ihre höhere Dosiseffizienz und zudem reduzierte Anfälligkeit für Artefakte ein großes Potential für die CT-Bildgebung (Kalender und Kyriakou 2007). Verglichen mit einem konventionellem Mehrschichtcomputertomographen (MSCT) bietet die FD-Technologie eine höhere Ortsauflösung und weniger Artefakte, jedoch geht sie mit einer niedrigeren Dosiseffizienz, einem kleineren field-of-view (FOV) und einer geringeren zeitlichen Auflösung einher (Obenauer et al. 2007, Kalender und Kyriakou 2007). Aufgrund dieser Eigenschaften finden Flachdetektoren zunehmend Verbreitung insbesondere in C-Bogen-FD-CT-Systemen, die zur interventionellen und intraoperativen Bildgebung eingesetzt werden. Eine neue hochauflösende computertomographische Technologie ist zudem die flächendetektorbasierte Volumen-CT (fpVCT) (Ning et al. 2000, Kalender 2003, Ning et al. 2003, Kiessling et al. 2004, Marten et al. 2004, Greschus et al. 2005, Heidrich et al. 2005 a), die mit einer Ortsauflösung von $140 \mu \mathrm{m}$ bzw. 3,6 Linienpaaren pro mm arbeitet und in dieser Studie als präklinisches diagnostisches Verfahren periimplantärer Strukturen evaluiert wurde. Im Rahmen der vorliegenden präklinisch-experimentellen Studie sollte evaluiert werden, wie detailgetreu auch kleinste periimplantäre Strukturen im Alveolarknochen, Fremdmaterialien und enossale Implantate mit der neuen Technologie der fpVCT zerstörungsfrei dreidimensional darstellbar sind. Die erhobenen Befunde wurden sowohl mit konventionellen zweidimensionalen Zahnfilmaufnahmen und Orthopantomographien als auch mit dreidimensionalen DVT-Aufnahmen verglichen.

\subsection{Die enossale Implantologie}

\subsubsection{Enossale Implantate}

Der heutige Einsatz von dentalen Implantaten erfolgt dann, wenn natürliche Zähne fehlen, die als Einzelzahnersatz oder als Pfeiler für eine prothetische Versorgung notwendig sind. Allein in Deutschland werden jährlich mehr als eine Million Implantate pro Jahr mit steigender Tendenz inseriert (DGI 2009). 
Voraussetzung für einen dauerhaften Erfolg enossaler Implantate ist die knöcherne Verankerung des Implantatkörpers durch unmittelbaren Kontakt der Implantatoberfläche mit dem periimplantären Knochen (Schliephake 2004). Dabei wird zwischen der schleimhautbedeckten, unbelasteten Einheilung mit prothetischer Versorgung nach mehreren Monaten und der offenen, früh belasteten Einheilung in den Knochen mit sofortiger Versorgung unterschieden (Stanford 2005). Beide Methoden haben gezeigt, dass sie äquivalente Ergebnisse in klinisch identischen Situationen erreichen (Jokstad et al. 2003). Wesentlich für die frühe Belastung sind dabei vor allem die primäre Stabilität des Implantates und die Qualität und Quantität des Knochens (Schliephake 2004), um Relativbewegungen zwischen Implantat und umgebendem Knochen zu vermeiden (Brunski 1992, Søballe et al. 1992). In einer Studie wurden Implantate mit bzw. ohne okklusalen Kontakt nach Insertion im Anschluss an eine Einheilungsphase von fünf Wochen klinisch und histologisch untersucht. Sowohl die klinische als auch die histologische Untersuchung ergaben keine Unterschiede hinsichtlich der Implantatstabilität, der klinisch erkennbaren Osseointegration und der histologischen sichtbaren Knochenreaktion (Degidi et al. 2009). Der derzeitige Trend geht jedoch zugunsten des Patienten zur Sofortversorgung. Weiter unterscheidet man die Sofortversorgung nach Spätimplantation (Implantation in ein allseits knöchern konsolidiertes Lager) von der Sofortversorgung nach Sofortimplantation (Implantation in die Alveole des entfernten Zahnes), wobei die sofortige Versorgung nicht immer synonym mit einer funktionellen Belastung ist (Schliephake 2004).

Anhand der Implantatart werden zylinderförmige Implantate mit und ohne Schraubgewinde von blattförmigen Extensionsimplantaten unterschieden. Zudem gibt es einteilige Implantatsysteme (Implantatkopf ragt nach Insertion unmittelbar aus der Schleimhaut heraus), die nur schwer eine Entlastung während der Einheilphase gewährleisten, und mehrteilige Implantatsysteme (bestehend aus dem im Knochen verankerten Körper, dem im Bereich der Mundschleimhaut liegenden Halsteil und dem Kopfteil, das die Suprakonstruktion trägt), die eine Entlastung während der Einheilungsphase ermöglichen (Branemark et al. 1977, Ericsson und Nilner 2002, Esposito et al. 2007, Testori et al. 2007). Ein Zahnarzt kann theoretisch zwischen mehr als 2000 verschiedenen Implantaten je nach Behandlungssituation des Patienten wählen (Jokstad et al. 2003). 
Derzeit geht die Studienlage dahin, neben den bisher aufgrund ihrer hohen Biokompatibilität verwendeten Titanimplantaten auch Implantate aus Zirkonoxidkeramik einzusetzen. Keramische Zirkonimplantate weisen eine vergleichende Erfolgsrate auf wie Titanimplantate und bieten eine gute Alternative für den künstlichen Zahnersatz, wobei ergänzende Untersuchungen weiterhin erstrebenswert wären (Oliva et al. 2007, Andreiotelli et al. 2009, Zembic et al. 2009). Einen entscheidenden Faktor für die Einheilung in den Knochen stellt neben dem Material auch die Oberflächenbeschaffenheit des Implantates dar. Für raue Zirkonoxidkeramikimplantate konnten Erfolgsraten nach 5-jähriger Behandlungsdauer von 95\% erreicht werden, wobei die dabei verwendeten säuregeätzten Oberflächen den beschichteten und unbeschichteten Oberflächen überlegen waren (Oliva et al. 2010). Mäßig aufgeraute Implantatoberflächen führen aufgrund ihrer Oberflächenvergrößerung zu einer stärkeren Osseointegration als stark aufgeraute oder glatte Oberflächenstrukturen (Albrektsson und Wennerberg 2004). Auch bei primär instabilen Implantaten kann eine hohe Überlebensrate erzielt werden, wenn das Implantat eine raue Oberflächenstruktur aufweist. Balshi et al. zeigten Überlebensraten von primär instabilen Implantaten mit angerauter Oberfläche von $91,7 \%$ und abgerundeten Oberflächen von 70\% (Balshi et al. 2007). Auch können verschiedene Faktoren, wie das weibliche Geschlecht, Insertionsorte außerhalb des anterioren Unterkiefers und eine Implantatlänge kürzer als $15 \mathrm{~mm}$ die primäre Implantatstabilität ungünstig beeinflussen (Mesa et al. 2008, Cooper 2010). Allgemein liegt die Erfolgsrate von Dentalimplantaten mittlerweile bei weit über $90 \%$ (Bahat 2000, Chung et al. 2009, Lambert et al. 2009, Oliva et al. 2010).

\subsubsection{Präoperative Diagnostik und Behandlungsplanung}

Heute werden in der Oral- und Mund-, Kiefer-, und Gesichtschirurgie immer häufiger Computer-gestützte Verfahren zu diagnostischen und therapeutischen Zwecken eingesetzt (Garg und Vicari 1995, Holberg et al. 2005, Loubele et al. 2007, Vandenberghe et al. 2007, Monsour und Dudhia 2008, Patel 2009, Patel et al. 2009). Zunehmend bedient man sich digitaler Bildgebungsverfahren, um sich anatomischstrukturelle und physiologische Gegebenheiten zu verdeutlichen. Zum Einsatz kommen hier neben den konventionellen radiologischen Verfahren der 
zahnmedizinisch-radiologischen Diagnostik die Computertomographie, die Magnetresonanztomographie und die Digitale Volumentomographie (Garg und Vicari 1995, Ekestubbe et al. 1997, Monsour und Dudhia 2008, Patel 2009, Patel et al. 2009). Indikationen für diese Art der radiologischen Darstellung sind neben der erweiterten Diagnostik der Einsatz in der dentalen Implantologie und in der Behandlungsplanung kraniofazialer Tumoren und Fehlbildungen.

Diesen modernen dreidimensionalen Möglichkeiten der Darstellung stehen die konventionellen zweidimensionalen Verfahren gegenüber, die auch heute noch aufgrund ihrer Zeichenschärfe und der geringen Strahlenbelastung ihren klar definierten Einsatzbereich in der Zahnmedizin innehaben. So findet z.B. der Zahnfilm seinen Einsatz in der feinen detailreichen radiologischen Diagnostik im Rahmen der Kariologie und Endodontie (Viktorov 2006).

Die Orthopantomographie hingegen findet ihre Indikation in der Darstellung von Übersichtaufnahmen des gesamten Kieferbereiches bei hoher Bildqualität. Sowohl Ober- und Unterkiefer, angrenzende basale Kiefer- und Nasennebenhöhlen und die Kiefergelenke können bildlich dargestellt werden. Dabei kommt das Verfahren mit einer insbesondere im Vergleich zu Zahnfilm und Computertomographie geringeren Strahlenbelastung für den Patienten aus (Kaeppler et al. 2000).

Prinzipiell wird zwischen der konventionellen Dentalradiologie und der digitalen Radiographie unterschieden (Youssefzadeh et al. 1999). Die Dentalradiologie bezeichnet die konventionellen radiologischen Verfahren in der Zahnmedizin, welche im klinischen Alltag durch den Zahnarzt selbst durchgeführt werden. Sie setzt sich zusammen aus der intraoralen Technik (Zahnfilme, Bissflügelaufnahmen, Aufbißaufnahmen) und der extraoralen Technik (Panoramaschichtaufnahmen, Fernröntgen). Die digitale Radiographie ermöglicht die Umgehung der konventionellen Entwicklungsverfahren, eine Strahlendosisreduktion und bietet die Möglichkeit der Bildverarbeitung (Youssefzadeh et al. 1999). Sowohl Zahnfilme als auch Orthopantomographien sind als digitale Verfahren verfügbar. Im Vergleich zu den analog arbeitenden konventionellen Radiologieverfahren weist die digitale Radiographie einige Vorteile bezüglich der Bildqualität und Datenverarbeitung auf. Neben einer besseren Bildqualität als das Film-Foliensystem zeigt sich eine höhere Orts-/Kontrastauflösung mit höherer Dosiseffektivität (Youssefzadeh et al. 1999). Ebenso kann eine digitale Bildverarbeitung, -befundung und -archivierung durch Anbindung an das digitale Bildarchivierungssystem PACS 
(Picture Archiving and Communication System) bzw. KIS/RIS-Anbindung (Krankenhaus- bzw. Radiologieinformationssystem) auf Grundlage von DICOMDatensätzen erfolgen, was einen schnelleren radiologischen Arbeitsfluss begünstigt. DICOM-Datensätze (Digital Imaging and Communication in Medicine) stellen ein standardisiertes Format zur Speicherung von Daten bereit, wodurch im klinischen Umfeld Interoperabilität zwischen Systemen verschiedener Hersteller erreicht wird (May et al. 2000, Imhof et al. 2002, De Backer et al. 2004).

Vor Durchführung einer Implantatinsertion liegt ein großes Augenmerk auf der Beurteilung der individuellen Kieferknochenverhältnisse. Die primäre ImplantatStabilität nach Insertion wird erreicht durch Kongruenz zwischen dem chirurgisch präparierten Alveolarbett und dem Implantat, was neben dem makroskopischen Implantatdesign und der chirurgischen Technik auch von der Knochendichte abhängig ist (Glauser et al. 2004, O'Sullivan et al. 2004, Sennerby und Meredith 2008). Es stellt sich vor allem die Frage nach ausreichend vorhandener Knochensubstanz und der räumlichen Beziehung des Implantationsbereiches zu umliegenden Strukturen wie benachbart verlaufender Nerven (N. alveolaris inferior und $\mathrm{N}$. lingualis) und der Ausdehnung der Kieferhöhle, um mögliche Komplikationen zu vermeiden (Theisen et al. 1990, Iplikcioglu et al. 2002, Auluck et al. 2007). Diese können vor allem neben der Traumatisierung umliegender Gewebe und Strukturen die Schädigungen des N. alveolaris inferior sein. Um Komplikationen dieser Art zu umgehen, ist es notwendig einen Sicherheitsabstand von mindestens 1 $\mathrm{mm}$ zwischen Implantat und Canalis mandibulae einzuhalten (Rubenstein und Taylor 1997). Voraussetzung dafür ist jedoch die eindeutige präoperative Identifikation des Canalis mandibulae, um die entsprechende Insertionstiefe vorherbestimmen zu können.

\subsection{Konventionelle Bildgebungsverfahren (Dentalradiologie)}

\subsubsection{Zahnfilm (ZF)}

Intraorale Röntgenbilder (Zahnfilme) erlauben die zweidimensionale Darstellung der Zähne und Kieferknochen in der vertikalen Ebene. Die intraorale Röntgentechnik eignet sich im Rahmen der oralen Implantologie für die initiale Beurteilung der 
vorliegenden Behandlungssituation (Frederiksen 1995). Sie ermöglicht Aussagen über das Vorliegen von Pathologien und eine Einschätzung der Qualität des Knochens. Zudem sind Beurteilungen über angrenzende anatomische Strukturen nahe dem Implantationsgebiet wie Wurzeln von Nachbarzähnen, den Sinus maxillaris und eine vorläufige Einschätzung der Knochenqualität möglich. Die exakte Bestimmung des vertikalen Knochenangebotes ist hingegen nur eingeschränkt möglich. Auch die präzise Lokalisation von anatomisch kritischen Strukturen, wie z.B. des Canalis mandibulae, kann im Rahmen der präoperativen Diagnostik nicht ausreichend beurteilt werden. Zudem sind keine exakten Aussagen über die Dimensionen des Alveolarfortsatzes in bukko-lingualer oder horizontaler Richtung möglich (Frederiksen 1995).

Allerdings hat dieses Verfahren eine sehr hohe räumliche Auflösung von $10-12$ Lp/mm (Wenzel 1995) und weist eine hohe Detailgenauigkeit von Zahnröntgenaufnahmen auf. Postoperativ kommen Zahnfilme vor allem bei Einzelzahnimplantaten zur Anwendung, um die periimplantären knöchernen Strukturen zu beurteilen und - projektionsbedingt mit Einschränkungen - zu kontrollieren (Gómez-Román et al. 1995, Keller 1995).

„Beim unbezahnten Patienten relativiert sich die Anwendung von Zahnfilmen in mancherlei Hinsicht" (Koeck und Wagner 2004, S. 30). Es ist schwierig den Film parallel zum Alveolarfortsatz bzw. zum Implantat korrekt zu positionieren (Frederiksen 1995). Dies gilt vor allen Dingen für die interforaminale Unterkieferregion. „Vertikale Knochen-/ Implantat-Relationen können so nicht beurteilt werden“ (Koeck und Wagner 2004).

\subsubsection{Orthopantomographie (OPG)}

Extraorale Röntgenbilder wie die Panoramaschichtaufnahme ermöglichen Übersichtsaufnahmen des gesamten Ober- und Unterkiefers einschließlich beider Kiefergelenke und der Kieferhöhlen und die Darstellung von Knochenstrukturen. Sie stellen ein schnelles und einfaches Verfahren zur präoperativen Diagnostik dar, sind kostengünstig und bedeuten eine geringe Strahlendosis für den Patienten (Vazquez et al. 2007). Oftmals wird dieses Verfahren in der oralen Implantologie eingesetzt, um die Implantatposition im Knochen prä- und postoperativ zu ermitteln (ten 
Bruggenkate et al. 1989). Diese Technik ermöglicht eine zweidimensionale Bildgebung des horizontalen und vertikalen Knochenangebotes in zahnlosen Kieferregionen, für die eine Implantatinsertion vorgesehen ist (Garg und Vicari 1995). Sie kann als ein sicheres Darstellungsverfahren im Rahmen der präoperativen Diagnostik für Routineimplantationen im posterioren Unterkieferbereich betrachtet werden (Vazquez et al. 2007). Das Vorliegen von Pathologien, die räumliche Beziehung zu anatomischen Strukturen, deren Bezug zum Alveolarfortsatz und eine Einschätzung des Knochenangebotes sind evaluierbar. Eine adäquate Beurteilung der Knochenqualität sowie -quantität ist jedoch nicht möglich (Frederiksen 1995). In den letzten 15 Jahren sind aus dem klassischen Orthopantomographen Multifunktionsgeräte geworden, die den größten Teil der radiologischen Fragestellungen aus der Zahn-, Mund- und Kieferheilkunde abdecken (Fuhrmann et al. 2003). Unterschieden werden dabei heute Geräte, die nach dem Prinzip der Panoramaschichttechnik arbeiten, von solchen, die aus einer Kombination von Panoramaschichtaufnahme und klassischer Tomographie arbeiten (Fuhrmann et al. 2003). Der in dieser Studie angewandte Orthopantomograph Orthophos (Sirona, Deutschland) arbeitet ausschließlich nach dem Panoramaschichtprinzip und verfügt zudem über Spezialeinstellungen für den Ober- und Unterkiefer, mehrere Kiefergelenkprogramme und Ablaufbahnen für das Mittelgesicht (Bschorer et al. 1993, Fuhrmann et al. 2003).

Die Orthopantomographie weist einen Verzerrungsfehler auf, der bei einer genauen Datenerhebung berücksichtigt werden muss (Frederiksen 1995). Es kommt zu einer charakteristischen Vergrößerung und Verzerrung der Abbildung, einhergehend mit einer Verminderung der Zeichenschärfe (Reddy et al. 1994). Anhand von Messschablonen kann dieser Verzerrungsmaßstab berechnet werden (Buser et al. 1990, Tal und Moses 1991, Hollender 1992).

Die verschiedenen Aufnahmen der konventionellen Röntgendiagnostik haben nachteilig die Eigenschaft, dass sie auf Grund ihrer Zweidimensionalität Summationsbilder der abgebildeten Region liefern. Sie sind hinsichtlich spezieller Details nur eingeschränkt aussagekräftig (Reddy et al. 1994, Monsour und Dudhia 2008).

Die Diagnostik und Therapie einer Reihe von zahnärztlich-chirurgischen Krankheitsbildern erfordert jedoch die Röntgendiagnostik in mehr als nur einer Ebene 
und somit eine überlagerungsfreie Schichtdiagnostik, um die zu beurteilende Region aussagekräftig darstellen zu können (Reddy et al. 1994).

\subsection{Dreidimensionale bildgebende Verfahren in der Zahn-, Mund- und Kieferheilkunde}

\subsubsection{Die Computertomographie (CT)}

1972 wurde die CT durch den amerikanischen Mediziner Allan M. Cormack und den britischen Ingenieur Godfrey N. Hounsfield entwickelt.

Der Beginn der Darstellung einer dritten Dimension des Kieferbereiches wurde zunächst anhand der Lineartomographie (Theisen et al. 1990) versucht. Aufgrund der jedoch Ende der 80er Jahre besseren Verfügbarkeit von Computertomographen und zudem verbesserten dentalen Software wurde diese Technologie schnell verdrängt (Rothman et al. 1988, Schwarz et al. 1989). Ursächlich dafür war zudem die unscharfe Darstellungsweise, wodurch die diagnostischen Möglichkeiten und der klinische Einsatz im oromaxillofazialen Bereich zu stark eingeschränkt waren (Butterfield et al. 1997).

Mit der Entwicklung der Spiralcomputertomographie im Jahre 1989, die ebenfalls 3DDarstellungen liefert, galt diese dann als neue, vor allem kostengünstigere Alternative zum herkömmlichen CT (Ekestubbe et al. 1996, Ekestubbe et al. 1997).

Die Spiralcomputertomographie generiert Datensätze, die durch gegenläufige Bewegung von Röntgenröhre und Film auf einer spiralförmigen Bahn rund um den im Focus liegenden zu untersuchenden Bereich konstruiert werden. Dadurch können annähernd orthoradiale Schichten akquiriert werden, die in der oralen Implantologie von Bedeutung sind (Solar und Gahleitner 1999). Orthoradiale Schichten (=Dentalschichten) und multiple Panoramaschichten können mit einem speziellen Dental-Softwareprogramm aus axialen Schichten berechnet werden (Abrahams 1999). Die Panoramaschichten werden parallel zu einer, auf einer axialen Schicht der Mandibula gezeichneten Kurve reformatiert; die Dentalschichten werden entlang nummerierter, senkrecht zur Planungslinie stehender Linien reformatiert. Anschließende Generierungen von 3D-Rekonstruktionen sind ebenso möglich. 
Dadurch können genaue Informationen über die Struktur des Kieferknochens und über Lage und Zustand der Zahnwurzeln gewonnen werden, wonach sichere Aussagen hinsichtlich Höhe und Breite des Kiefers, Knochendichte, Lage des Foramen mentale, des Alveolarkanals bzw. des N. alveolaris inferior sowie der Dicke des darüber liegenden Knochens und der Lagebeziehung zu den Nasennebenhöhlen möglich sind (Schwarz et al. 1987 a, Schwarz et al. 1987 b).

In den folgenden Jahren kam es zu einer enormen Weiterentwicklung der CTTechnologie. Zunächst wurden in den 90er Jahren immer höhere Detektorzeilenzahlen entwickelt, die eine Steigerung der Volumenaufnahmegeschwindigkeit um den jeweiligen Faktor der Zeilenzahl ermöglichten (Kalender 2003). Seit 1998 stehen der klinischen Radiologie sogenannte Mehrschicht-Spiral-Computertomographen (MSCT) zur Verfügung. Hierbei sind mehrere Detektorzeilen nebeneinander angeordnet, so dass mit einer Röhrenumdrehung mehr als eine Untersuchungsschicht simultan errechnet werden kann (Kalender 2003). Diese Schichten resultieren aus der Festlegung gleichzeitig erfasster Schichten und nicht aus der Zahl der Detektorzeilen.

Die Vierschicht-CT galt als ein grundlegender Fortschritt in der Entwicklung und fortlaufenden Verfeinerung der CT-Bildgebungsverfahren. Die Generation der Vierschicht-CT-Systeme wurde schnell von Sechs-, Acht- und Sechzehn-SchichtCT-Systemen abgelöst. Bereits seit 2003 werden 64-Zeilen-Geräte zur klinischen Diagnostik am Menschen verwendet. Bislang nur zu Forschungszwecken sind mittlerweile Zeilengeräte mit bis zu 256 Zeilen (Prototypen) für die präklinische Diagnostik entwickelt worden (Kalender 2003).

Durch die vergrößerte Zeilenzahl wurden nun routinemäßig Dünnschichtaufnahmen für größere Aufnahmebereiche eingesetzt, wodurch in deutlich kürzerer Zeit eine erheblich verbesserte axiale Auflösung und bessere Nutzung der Röntgenröhre erzielt werden konnte (Ohnesorge et al. 1999, Kalender 2003). Daraus ergibt sich eine verkürzte Untersuchungszeit mit einer lückenlosen Erfassung von größeren Volumendatensätzen (Ohnesorge et al. 1999). Vorteilig ist zudem die Reduktion von Partialvolumeneffekten durch Rekonstruktion überlappender Schichten und die Möglichkeit der dreidimensionalen Rekonstruktion mit Berechnung von koronaren, sagittalen oder irregulären Schichtebenen. Das größte Potential liegt jedoch in der Verringerung der Schichtdicke und der damit verbundenen Verbesserung der axialen Auflösung, was von verschiedenen Autoren als die wichtigste Neuerung der 
Mehrschichtigkeit beschrieben wird (Ohnesorge et al. 1999, Böhme et al. 2003, Bitschnau et al. 2004, Schima et al. 2005).

Im Gegensatz zur OPG, die eine vergleichsweise hohe Schichtdicke aufweist und daher mit einer Überlagerung von Nachbarstrukturen einhergehen kann, ermöglicht die Mehrschicht-Dental-CT eine überlagerungs- und verzerrungsfreie Abbildung sowie Vermessung der Strukturen mit einer Darstellungsgenauigkeit von deutlich unter einem Millimeter (Hirschfelder 1994, Reddy et al. 1994, Solar und Gahleitner 1999, Webber und Messura 1999, Nance et al. 2000, Gahleitner et al. 2003, Flohr et al. 2005 b). Moderne klinische CT-Geräte setzen mit Ortsauflösungswerten von teilweise mehr als $0,5 \mathrm{~mm}$ in der Aufnahmeebene und in beliebigen multiplanaren Darstellungen den Goldstandard. Werte für den klinischen Betrieb eines 4-SchichtCT-Gerätes liegen im Bereich von 0,6 - 0,8 mm (Kalender 2003, Flohr et al. 2005 a), das auflösungsbestimmende Bildvoxel ist dabei ca. $0,5 \times 0,5 \times 1,0 \mathrm{~mm}$ groß und damit noch nicht vollständig isotrop (Flohr et al. 2005 a). 16-Schicht-CT-Geräte erreichen eine Auflösung von $0,5 \mathrm{~mm}$ bei Standardabdomen- oder Thoraxuntersuchungen, wobei mit einer Auflösung von 0,6 mm senkrecht dazu das Ideal der isotropen Auflösung erreicht werden kann. 64-Zeilen-CT-Geräte erreichen ebenso mindestens eine Ortsauflösung von 0,5 mm (Rogalla et al. 2009). Auflösungswerte von 0,33 mm (15 Linienpaare pro $\mathrm{cm}$ ) konnten mit einem Hochauflösungs-Gitterphantom nachgewiesen werden (Flohr et al. 2004). Im Rahmen urographischer Untersuchungen an Phantomen waren Füllungsdefekte mit einer Größe von 0,25 mm darstellbar (Vrtiska et al. 2009). Dabei wurden anhand von Uretermodellen mit Hilfe von Kontrastmittel sowohl Defekte bei der Füllung der Ureter als auch Divertikel im Ureter dargestellt.

Durch die Möglichkeiten der hochauflösenden CT und die Entwicklung entsprechender Software zur Darstellung des Kiefers in räumlich zuordenbaren Schichtebenen, lassen sich auch kleinste, zahnärztlich relevante Strukturen und ihre Lagebeziehung zueinander in allen drei Ebenen darstellen. Durch den Einsatz hochauflösender Dental-CT können selbst Strukturen im Millimeterbereich, wie z.B. der N. alveolaris inferior, suffizient dargestellt werden. 


\subsubsection{Digitale Volumentomographie (DVT)}

Bei der DVT handelt es sich um eine digitale Aufnahmetechnik, bei der ein dreidimensionales Strahlenbündel und ein Flächendetektor verwendet werden. Auf einer zirkulären Bahn wird eine große Anzahl von Projektionsaufnahmen erzeugt, aus welchen unmittelbar ein 3D-Volumen des zu untersuchenden Körperteils berechnet wird. Für das Verfahren typisch sind eine isometrische Ortsauflösung im Volumen in allen drei Raumrichtungen sowie die Konzentration auf die Darstellung von Hochkontrast, d.h. auf Hartgewebe. Gegenüber der klassischen (Einzeilen-) Computertomographie grenzt sich die DVT durch die Verwendung eines dreidimensionalen Nutzstrahlenbündels (Cone Beam) sowie eines zweidimensionalen Bildrezeptors ab (DGZMK 2009).

Seit 1997 steht der Zahn-, Mund- und Kieferheilkunde die Digitale Volumentomographie als neues Untersuchungsverfahren der zahnärztlichchirurgischen Diagnostik zur Verfügung. Seitdem führt dieses Verfahren nicht nur zu einer deutlichen Erweiterung der radiologischen Möglichkeiten sondern auch zu einer zunehmenden Verbreitung in den letzten Jahren, da sowohl die Durchführung als auch die Befundung durch den Zahnarzt selbst erfolgen kann.

Die zahnärztlichen Indikationen umfassen gemäß einer Stellungnahme der DGZMK folgende Teilbereiche (DGZMK 2009):

- Endodontie:

- apikale Veränderungen bei Vorliegen klinischer Auffälligkeiten; an Molaren und Prämolaren sowohl des Ober- als auch des Unterkiefers konnten mit dem 3D Accuitomo periapikale Pathologien und das Wurzelkanalsystem detailliert dargestellt werden (Lofthag-Hansen et al. 2007), auch iatrogen gesetzte periapikale Defekte waren evaluierbar (Stavropoulos und Wenzel 2007).

- Wurzelfrakturen; diese können mit dreidimensionalen Verfahren sicherer identifiziert werden. Mit Hilfe eines dreidimensionalen FlächendetektorVolumencomputertomographen waren vertikale Wurzelfrakturen in drei Ebenen evaluierbar (Hannig et al. 2005) ebenso konnten gute Ergebnisse mit dem 3D Accuitomo erreicht werden (Bernardes et al. 2009).

- Wurzelresorptionen, z.B. nach Zahntrauma (Cohensca et al. 2007 a) 
- Parodontologie:

- dreidimensionale Visualisierung der knöchernen Parodontalmorphologie; peridontale Knochendefekte an menschlichen Schädeln, deren Morphologie und eine Größenbestimmung waren mit der DVT evaluierbar (Vandenberghe et al. 2007), ebenso die exakte überlagerungs- und verzerrungsfreie Bestimmung anatomischer Strukturen in drei Ebenen (Mengel et al. 2005, Farman und Scarfe 2006)

- zahnärztliche Prothetik: Visualisierung des qualitativen und quantitativen Knochenangebotes, Darstellung von Nervenaustrittspunkten, Diagnostik von knöchernen Kiefergelenkerkrankungen (Fu et al. 2007, Honey et al. 2007, Hussain et al. 2008), Virtuelle Planung von implantatprothetischen Versorgungen (Ganz 2008) - chirurgische Zahnheilkunde: Wurzelfrakturen (Cohenca et al. 2007 b), Alveolarfortsatzfrakturen (Cohenca et al. 2007 b), größere periapikale knöcherne Läsionen (Simon et al. 2006)

- Implantologie: dreidimensionale Darstellung des Implantationsgebietes im Rahmen präoperativer Behandlungsplanung (Garg und Vicari 1995, Monsour und Dudhia 2008) und die präoperative Bestimmung der Knochendichte (Aranyarachkul et al. 2005)

- Mund-, Kiefer- und Gesichtschirurgie

- Kieferorthopädie (Müssig et al. 2005).

Konventionelle zweidimensionale radiologische Untersuchungsverfahren haben gezeigt, dass sie aufgrund ihrer Zweidimensionalität nur begrenzt Informationen bei endodontischen Fragestellungen liefern. Hier hat sich die CBCT als sicheres und effektives Darstellungsverfahren erwiesen (Patel et al. 2009).

Die DVT oder CBCT liefert als extraorales Verfahren dreidimensionale Datensätze der maxillofazialen Region und zeigt zudem eine deutlich höhere Strahlendosiseffizienz als herkömmliche Computertomographen (Mozzo et al. 1998, Arai et al. 1999). Die CBCT unterscheidet sich von CT dadurch, dass ein gesamter 3D-Volumendatensatz durch eine einzelne Umdrehung des Scanners akquiriert werden kann. Der Röntgenstrahl der CBCT ist kegelförmig und erfasst einen zylindrischen oder kugelförmigen Volumendatensatz, der als field-of-view (FOV) beschrieben wird. Die Volumina der verschiedenen Systeme unterscheiden sich auch im Auflösungsvermögen, das über das Volumenelement (=Voxel) definiert ist. Die Voxelgröße schwankt dabei typischerweise zwischen $0,08 \mathrm{~mm}^{3}$ und $0,4 \mathrm{~mm}^{3}$ (Patel 
et al. 2009). Die klinisch angewandten DVT-Geräte (siehe auch Tabelle 1) wie der 3D Accuitomo und der NewTom QR-DVT 9000 haben eine Voxelgröße von 0,125 mm (3D Accuitomo) (Loubele et al. 2007) bzw. von 0,25 mm x 0,25 mm x 0,2 mm (NewTom QR-DVT 9000) (Lernkamp 2006) (Tab. 1). Der ProMax 3D hat isotrope Voxel von $160 \times 160 \times 160 \mu \mathrm{m}$.

\begin{tabular}{|c|c|c|c|c|}
\hline DVT & $\begin{array}{l}\text { 3D Accuitomo } \\
\text { (Morita, Kyoto, } \\
\text { Japan) }\end{array}$ & $\begin{array}{c}\text { NewTom } \\
\text { QR-DVT } 9000 \\
\text { (Verona, Italien) }\end{array}$ & $\begin{array}{c}\text { Planmeca } \\
\text { ProMax 3D } \\
\text { (Helsinki, } \\
\text { Finnland) }\end{array}$ & $\begin{array}{c}\text { I CAT } \\
\text { (Imaging Sciences, } \\
\text { Hatfield, } \\
\text { Pennsylvania, } \\
\text { USA) }\end{array}$ \\
\hline Detektor & Flat Panel & $\begin{array}{l}\text { Image intensifier } \\
(\mathrm{IB})+\mathrm{CCD} \text { camera } \\
+ \text { digitize }\end{array}$ & $\begin{array}{l}\text { Flat panel } \\
624 \times 624, \\
\text { element } \\
200 \times 200 \mu \mathrm{m}^{2}\end{array}$ & $\begin{array}{l}\text { Flat Panel } \\
20 \times 25 \mathrm{~cm}\end{array}$ \\
\hline X-Ray & $\begin{array}{l}\text { Cone Beam / } \\
\text { Pyramide }\end{array}$ & Cone Beam & Pyramide & Zylinder \\
\hline Scan-Zeit & $18 \mathrm{~s}$ & $75 \mathrm{~s}$ & $18 \mathrm{~s}(1949$ & $5 ; 8,9 ; 26,9 \mathrm{~s}$ \\
\hline $\begin{array}{c}\text { FOV } \\
\text { (field-of-view) }\end{array}$ & $\begin{array}{l}40 \times 40 \mathrm{~mm} \\
60 \times 60 \mathrm{~mm}\end{array}$ & $\max .305 \times 305 \mathrm{~mm}$ & $\begin{array}{l}40 \times 50 \mathrm{~mm}, \\
80 \times 50 \mathrm{~mm}, \\
80 \times 80 \mathrm{~mm}\end{array}$ & $\begin{array}{l}4,6,8,10,13 \mathrm{~cm} \mathrm{x} \\
16 \mathrm{~cm}\end{array}$ \\
\hline Voxelgröße & $\begin{array}{l}0,125 \mathrm{~mm}^{3} \\
(10 \% \mathrm{MTF})\end{array}$ & $\begin{array}{l}0,25 \mathrm{~mm} \times \\
0,25 \mathrm{~mm} \times 0,2 \mathrm{~mm}\end{array}$ & $0,16 \mathrm{~mm}^{3}$ & $0,2-0,4 \mathrm{~mm}^{3}$ \\
\hline $\begin{array}{c}\text { Effektive } \\
\text { Strahlendosis }\end{array}$ & $\begin{array}{c}\text { 20,02 } \mu \text { Sv bis } \\
43,27 \mu S v \text { (Hirsch } \\
\text { et al. 2008) bzw. } \\
18 \mu \text { Sv bis } 66 \mu S v \\
\text { (Okano et al. 2009) }\end{array}$ & $56,5 \mu \mathrm{Sv}$ & $\begin{array}{l}24,3 \mu \text { Sv } \\
\text { (Ludlow et al. } \\
2008 \text { ) }\end{array}$ & $\begin{array}{l}36-74 \mu S v \\
(101,5 \mu S v)\end{array}$ \\
\hline
\end{tabular}

Tab. 1: Hersteller, Detektorart, Röntgenstrahl, Scan-Zeit, Field-of-view (FOV), Voxelgröße und effektive Strahlendosis der gängigen DVT-Geräte

Im Vergleich zur CT (Voxelgröße 0,4 mm Kantenlänge) haben die DVT ein höheres Auflösungsvermögen, woraus eine bessere Detaildarstellung einzelner Strukturen wie Knochen, Schmelz, Dentin, Pulpakavum und Parodontalspalt resultiert (Arai et al. 1999, Honda et al. 2001, Hashimoto et al. 2003). Die Größe des FOV ist dabei mit der Strahlendosis für den Patienten assoziiert (Hirsch et al. 2008, Okano et al. 2009, Roberts et al. 2009). Verringert man die Größe des Röntgenstrahls auf die kleinste 
notwendige Größe, um das relevante Objekt zu erfassen, so kann damit die Dosis für den Patienten deutlich reduziert werden (Hirsch et al. 2008).

CBCT sind im Gebrauch leicht anwendbar und decken denselben Bereich ab wie Panoramaschichtaufnahmen, wodurch sie sie sich gut als Verfahren in der Zahn-, Mund- und Kieferheilkunde eignen (Scarfe et al. 2006).

Sie bieten eine bessere Bildqualität als Spiral-CT bei Untersuchungen von Hartgewebsstrukturen (Hashimoto et al. 2003, Hashimoto et al. 2006, Hashimoto et al. 2007). Es konnte ebenso gezeigt werden, dass CBCT im Vergleich zu MSCT eine höhere Auflösung bei der Darstellung von kleinen Hochkontraststrukturen (Hartgewebe) wie z.B. Nervenkanälen mit den darin enthaltenen neurovaskulären Bündeln erreichen (Bartling et al. 2007). Nachteilig zeigt sich hingegen die geringere Kontrastauflösung von Weichgewebsstrukturen (Loubele et al. 2007).

Aufgrund der guten Darstellung von Hochkontraststrukturen findet die DVT neben dem Einsatz in der Implantologie Anwendung in der Endodontie und Parodontologie. Bereits in verschiedenen Studien konnten ihre Vorteile beschrieben werden. Das Ausmaß periimplantärer Defekte und parodontalen Knochenverlustes lässt sich mit der DVT mit nur geringen Abweichungen exakt darstellen, wobei dreidimensionale maßstabsgetreue Darstellungen ohne Überlappungen und Verzerrungen möglich sind (Mengel et al. 2005, Mengel et al. 2006). In einer Vergleichsstudie zur Darstellbarkeit von peridontalen Knochendefekten zeigten die DVT-Daten im Vergleich zu intraoralem Zahnfilm, Orthopantomographie und der CT die beste Bildqualität (Mengel et al. 2005). Die DVT eignet sich ebenso zur Darstellung und genauen Größenbestimmung von parodontalem Knochenverlust (Vandenberghe et al. 2008) und ermöglicht eine morphologische Beurteilung von Knochendefekten (Vandenberghe et al. 2007). Sie liefert insgesamt bessere diagnostische und quantitative Informationen über den parodontalen Knochengehalt als konventionelle radiologische Verfahren ( $\mathrm{Mol}$ und Balasundaram 2008). Auch für die Darstellung und Diagnostik von Wurzelfrakturen ist die DVT ein ausgezeichnetes Untersuchungsverfahren (Bernardes et al. 2009, Kamburoğlu et al. 2009). Derzeit bieten verschiedene Hersteller DVT-Geräte an; der NewTom QR-DVT 9000 (Verona, Italien), Accuitomo 3D (Morita, Kyoto, Japan) und Planmeca ProMax 3D (Helsinki, Finnland) seien als Beispiele genannt (Tabelle 1). Sie unterscheiden sich vor allem hinsichtlich der Detektortechnologie, des FOV (field-of-view), der Dauer der Belichtungszeit und ihrer Auflösung. Gute Ergebnisse können im Bereich der 
Endodontie mit dem Accuitomo erreicht werden (Nakata et al. 2006, Bernardes et al. 2009, Kamburoğlu et al. 2009, Nakata et al. 2009). Für den ProMax 3D liegen ebenso gute Ergebnisse für den Einsatz in der Oralchirurgie und Implantologie vor (Rugani et al. 2009).

Mit dem NewTom QR-DVT 9000 konnten exakte Beurteilungen im Rahmen präoperativer implantologischer Behandlungsplanungen getroffen werden (King et al. 2007).

\subsubsection{Die flatpanel-Volumencomputertomographie (fpVCT)}

Eine neuartige hochauflösende computertomographische Technologie ist die flatpanel-Volumencomputertomographie (fpVCT) (Ning et al. 2000, Kalender 2003, Ning et al. 2003, Kiessling et al. 2004, Marten et al. 2004, Greschus et al. 2005, Heidrich et al. 2005 a), die mit Flachbilddetektoren (FD) arbeitet und damit immer mehr die analogen Film-Folien-Systeme und digitalen Speicherfolien ersetzt (Spahn et al. 2003). Während in den letzten Jahren ein deutlicher Fortschritt der Computertomographie in Bezug auf die zeitliche und räumliche Auflösung erfolgte, kam es parallel zu der Entwicklung von digitalen Flachbilddetektoren für konventionelle Röntgengeräte und Mammographien, wodurch eine ultrahohe Ortsauflösung $(150 \mu \mathrm{m} \times 150 \mu \mathrm{m})$ in zweidimensionalen Untersuchungen erreicht werden konnte (Gupta et al. 2006). Im Zuge der technologischen Weiterentwicklung werden immer häufiger die über lange Jahre benutzten Bildverstärkersysteme durch Flachdetektoren als bildgebendes Medium ersetzt (Spahn 2005). Neben der geometrisch verzerrungsfreien Objektdarstellung liegt ein weiterer Vorteil der FD gegenüber konventionellen Bildverstärkersystemen in einer deutlich höheren Kontrastauflösung (Kalender 2003). Im Flachdetektor-Volumen-CT sind die Vorteile der CT mit der digitalen Flachdetektor-Technologie kombiniert (Gupta et al. 2006). In der Entwicklung von Flachbilddetektoren wird eine zusätzliche Verbesserung der 3D-Ortsauflösung gesucht (Kalender 2003). Diese Detektoren können in wesentlich geringerer Größe hergestellt werden und so zu einer deutlich gesteigerten isotropen Ortsauflösung führen. Auflösungswerte von kleiner als $200 \mu \mathrm{m}(2,5 \mathrm{Lp} / \mathrm{mm})$ können erreicht werden; Spitzenwerte der klinischen CT liegen hingegen bei $500 \mu \mathrm{m}$ (Riedel 2005). Verglichen mit der Multi-slice-CT übertrifft die räumliche Auflösung der fpVCT 
(3 Linienpaaren/mm) deutlich die örtliche Auflösung der MSCT (1,2-1,4 Linienpaare/mm) (Kalender und Kyriakou 2007). Weiter konnten für die fpVCT Maximalwerte von $140 \mu \mathrm{m}(3,6 \mathrm{Lp} / \mathrm{mm}$ ) beschrieben werden (Heidrich et al. $2005 \mathrm{a}$, Kiessling et al. 2004). Durch die höhere Dosiseffizienz und reduzierte Anfälligkeit für Artefakte besitzen die FD-basierten Systeme ein großes Potential für die CTBildgebung (Heidrich et al. 2005 a, Kalender und Kyriakou 2007). Es wurden bereits verschiedene präklinische Untersuchungen mit dieser Technologie in der Endodontie durchgeführt, die den Vorteil der hohen Ortsauflösung untermauern. Selbst kleinste Strukturen wie Seitenkanäle können im Rahmen der präklinischen, zerstörungsfreien Evaluation der Wurzelkanalanatomie menschlicher Zähne detektiert und beurteilt werden (Heidrich et al. 2005 a). Potentiell lässt sich damit die fpVCT auch für die Diagnostik und Beurteilung der einzelnen Schritte in der Wurzelkanalbehandlung einsetzen (Heidrich et al. 2005 a). Außerdem ermöglicht sie neben der genauen dreidimensionalen Darstellung von Dentin, Schmelz und Wurzelkanalsystem die zerstörungsfreie Volumetrie dieser Teilgewebe des Zahnes (Hannig et al. 2006). Einzelne Strukturen und deren deutliche Unterscheidung waren dreidimensional aus allen Richtungen einsehbar und evaluierbar (Hannig et al. 2006). Präzise Aussagen über vertikale Zahnwurzelfrakturen sind zudem möglich (Hannig et al. 2005). Des Weiteren bietet die fpVCT ein hohes Potential zur Detektion und Differenzierung früher Stadien äußerer Wurzelresorptionshöhlen, die mit pathologischer Relevanz einhergehen (Hahn et al. 2009 b). Verglichen mit der MSCT war auch die Visualisierung der mittleren Gaumennaht durch die fpVCT aufgrund der hohen Ortsauflösung signifikant besser (Hahn et al. 2009 a). Zudem sind Untersuchungen im Rahmen von Tumordiagnostik wie das Aufsuchen und die Progression von osteolytischen Knochenmetastasen (Missbach-Guentner et al. 2007) und der Tumorangiogenese möglich (Kiessling et al. 2004). Auch ist die Visualisierung der menschlichen Schädelbasis im Rahmen maxillofazialer Untersuchungen möglich (Bartling et al. 2007).

Die potenzielle Verbesserung der Ortsauflösung der Flächendetektor-CT ist jedoch mit erhöhten Dosisanforderungen verbunden (Kalender 2003), so dass ein potenziell klinischer Einsatz am Menschen sorgfältig abgewogen werden muss. Um einen erheblichen Dosisanstieg zu vermeiden muss im Gegenzug dazu ein erhöhtes Bildrauschen mit verringerter Kontrastauflösung und geringerer Weichteildifferenzierung hingenommen werden (Kalender 2003). Folglich sind 
Entwicklungen mit Flächendetektoren besonders für die diagnostischen Bereiche interessant, die große Anforderungen an eine hohe Ortsauflösung stellen. Die im Vergleich zu konventionellen Bildverstärkersystemen deutlich bessere Kontrastauflösung schafft jedoch die technischen Vorraussetzungen, bis zu einem gewissen Grad Weichteildarstellungen zu ermöglichen. CT-ähnliche Bilder zur Weichteildarstellung können akquiriert werden (Ross et al. 2006). Insgesamt ist jedoch die Niedrigkontrastdarstellung aufgrund vermehrten Bildrauschens geringer als bei einem MSCT (8-SCT) (Funke et al. 2005).

Die Kombination von Flachbilddetektoren mit ausreichender Bildqualität und schnellen Rotationsgeschwindigkeiten bieten ein erfolgversprechendes technisches Konzept für medizinische CT-Systeme.

Flachbilddetektoren stellen die Basis für ein direkt elektronisch auslesbares digitales Radiographiesystem dar, wodurch das Verfahren auch als Direktradiographie bezeichnet wird (Kotter und Langer 2002). Im Gegensatz zu anderen Systemen sind Flachdetektoren durch eine aktive Matrix aus elektronischen Schaltelementen charakterisiert, die die gesamte Detektorfläche abdecken und jeweils einem Schaltelement ein Pixel des entsprechenden digitalen Bildes zuordnen. Der Vorteil dabei ist die verbesserte Effizienz der Umsetzung des absorbierten Strahlenreliefs in digitale Bilddaten und deren Weiterleitung (Schaefer-Prokop et al. 2003). Bei Flachbilddetektoren unterscheidet man je nach Konversion der Röntgenstrahlung Detektoren mit direkter bzw. indirekter Konversion (Spahn et al. 2003). Bei der indirekten Konversion werden zunächst die Röntgenquanten in einem Szintillator aus Gadoliniumoxisulfit oder Cäsiumjodid (CsJ) in Licht umgewandelt und in einem 2. Schritt das Licht in einer Photodiode aus amorphem Silizium in elektrische Ladung konvertiert. Bei der direkten Konversion erfolgen die Umwandlung der Röntgenstrahlung in Ladung und der Transport der gewonnenen Ladung zur Elektrode, was mit Hilfe eines elektrischen Feldes erfolgt, in einem Direktkonverter wie z.B. aus amorphem Selen (a-Se) (Spahn et al. 2003). Die in dieser Studie verwendeten Detektoren basieren auf dem Prinzip der indirekten Konversion und damit auf Szintillatoren aus Cäsiumjodid (CsJ) und Photodioden aus amorphem Silizium (a-Si). Dieses System hat den Vorteil, dass beide Prozessschritte weitgehend unabhängig voneinander optimiert werden können (Spahn et al. 2003). Konkurrierende digitale Radiographiesysteme stellen CCD-Systeme und die Speicherfolienradiographie dar. CCD-Detektoren (charge coupled device) werden 
nicht zu den flat-panel-Detektoren gerechnet. Wie in den Direktradiographiesystemen mit indirekter Konversion werden die Röntgenstrahlen mit Hilfe einer

Szintillatorschicht in Licht umgewandelt. Allerdings wird das Licht dann mit Hilfe eines Lichtleiters auf die CCD-Sensoren geleitet und dort in elektrische Signale umgewandelt (Schaefer-Prokop et al. 2003). CCDs sind Bildsensoren, bei denen die aktive Empfängerfläche in einzelne Pixel aufgeteilt ist. Gut einsetzbar sind diese Systeme für kleine Bildausschnitte, z.B. in der Skelettdiagnostik (Spahn et al. 2000). Alternativ zu diesen Verfahren stehen die Speicherfoliensysteme zur Verfügung. Mittlerweile konnten die DQE-Werte (Quanteneffizienz) über ein weites Spektrum von Ortsfrequenzen deutlich angehoben werden, so dass sie mit den Werten von Direktdetektorsystemen vergleichbar sind (Schaefer-Prokop et al. 2003).

Die neue Detektortechnologie der fpVCT hat verglichen mit der MSCT einige Vorteile. Dazu gehören die ultrahohe Ortsauflösung, die Echtzeit-Durchleuchtung, die Option dynamischer Untersuchungen und die Erfassung eines ganzen Organs innerhalb einer Umdrehung (Kalender und Kyriakou 2007, Gupta et al. 2008). Nachteilig hingegen sind die zur MSCT vergleichbar höhere Strahlendosis, die niedrigere Kontrastauflösung und die längere Scanzeit (Gupta et al. 2008). FpVCT-Untersuchungen der maxillofazialen Region haben in den letzten Jahren zunehmend an Aufmerksamkeit hinsichtlich bildgesteuerter Operationsverfahren in der Mund-, Kiefer- und Gesichtschirurgie, insbesondere der Implantologie, gewonnen. Obwohl die Relation der Bildqualität zu der Patientendosis unterhalb der eines MSCT liegt, geht das Bestreben in die Richtung, die fpVCT weiter in die Mund-, Kiefer-, Gesichtschirurgie zu integrieren (Kalender und Kyriakou 2007). Aufgrund ihrer hervorragenden isotropen Ortsauflösung zeigt sie deutliche Vorteile im Vergleich zur MSCT und bietet sich neben der Kleintierbildgebung insbesondere auch zur Darstellung von feinen knöchernen Strukturen und Implantaten an (Obenauer et al. 2005). Limitierend für die klinische Anwendung ist derzeit jedoch die fehlende variable Einblendung des Röntgenstrahles (Heidrich et al. 2005 a), so dass der Einsatz der fpVCT derzeit auf die experimentelle Medizin, insbesondere auf die Kleintierbildgebung beschränkt ist (Funke et al. 2005). Im präklinisch-experimentellen Einsatz stellt dieses Verfahren hingegen ein innovatives diagnostisches Verfahren zur zerstörungsfreien, dreidimensionalen Analyse von Zähnen dar (Heidrich et al. 2005 a). 
Seit Februar 2004 steht der Abteilung Diagnostische Radiologie der Universität Göttingen ein Flächendetektorbasierter-Volumen-Computertomograph (fpVCT) mit zwei Flächendetektoren aus amorphem Silizium in Form eines Prototyps zur Verfügung. Dieser Prototyp ist eine Entwicklung des General Electric (GE) Global Research Centers (Niskayuna, NY, USA) und wird sowohl im Tierexperiment als auch an histologisch-anatomischen Präparaten eingesetzt.

\subsection{Strahlenbelastung}

Die in der heutigen Zahn-, Mund- und Kieferheilkunde mittlerweile immer häufiger eingesetzten Computer gestützten radiologischen Verfahren bringen eine unterschiedlich hohe Strahlenbelastung mit sich. Es ist folglich vor jeder Anwendung gründlich abzuwägen, welchen effektiven Nutzen dieses Verfahren im Vergleich zu anderen radiologisch einsetzbaren Verfahren mit sich bringt, so dass eine strenge klinische Indikation gestellt werden muss.

Mittels des CTDI (CT-Dosisindex) erfolgt eine Abschätzung der Dosisbelastung der CT (Brix et al. 2003, Shope et al. 1981). Der CTDI wird aus dem Integral des Dosisprofils einer Einzelschicht im Phantomzentrum und 7 kranial bzw. kaudal angrenzenden Schichten ermittelt (Loewenhardt und Müller 2006). Diese Werte können auf Wasser- und Weichgewebe übertragen werden. Ein gemittelter Dosiswert resultiert aus einer Multicenterstudie der DRG und addiert 1/3 des Dosisprofils im Zentrum mit 2/3 im Randbereich der Phantommessung (Loewenhardt und Müller 2006). Ein gewichteter CTDIw berücksichtigt in Verbindung mit dem Dosislängenprodukt (DLP), der Strahlungsqualität und bestimmter Filterungen am ehesten die bauartbedingten, technischen dosisrelevanten Einflussgrößen Range (Scanlänge) und Pitch (Tischvorschub). Durch eine Modifikation im CTDlvol soll die bei MSCT-Untersuchungen anfallende Gesamtbelastung unter Einbeziehung der Strahlenbelastung zu Beginn und Ende des Untersuchungsvolumens („Overbeaming“) berücksichtigt werden (Loewenhardt und Müller 2006). Basierend auf Untersuchungen zur Strahlenexposition des Mittelgesichts lässt sich beispielsweise für eine Panoramaschichtaufnahme eine mittlere Energiedosis von 0,4 - 0,5 mGy errechnen (Rother 2001), andere Studien ergaben Maximaldosen von 0,25, 0,61 und 0,65 mGy (Lecomber et al. 2000, Lecomber et al. 2001). Vergleicht 
man die effektive Dosis von analogen und digitalen Panoramaschichtaufnahmen, so können deutlich niedrigere Werte mit den digitalen Verfahren erreicht werden (Visser et al. 2000). Die effektive Dosis einer digitalen Panoramaschichtaufnahme schwankt zwischen 4,7 bis $14,9 \mu \mathrm{Sv}$ pro Scan (Gijbels et al. 2005) bzw. 5 bis $14 \mu \mathrm{Sv}$ (Visser et al. 2000). Weitere Untersuchungen ergaben Werte von $8 \mu$ Sv bis $38 \mu S v$ (inklusive der Dosisbelastung für Speicheldrüsen) (Gavala et al. 2009). Für analoge Panoramaschichtaufnahmen wurden Werte von $17 \mu$ Sv bzw. $26 \mu$ Sv (inklusive der Dosisbelastung für Speicheldrüsen) (Gavala et al. 2009) bzw. 16 bis $21 \mu$ Sv (Visser et al. 2000) erreicht. Im Vergleich zu Panoramaschichtaufnahmen gehen intraorale Zahnfilmaufnahmen mit einer geringeren Strahlendosisbelastung mit Werten von 4 bis $8 \mu \mathrm{Sv}$ einher (Okano et al. 2009). Die mittlere Energiedosis einer Digitalen Computertomographie liegt bei $5 \mathrm{mGy}$, die einer MSCT mit bereits adaptierten Expositionsparametern bei 10,5 mGy (Möbes et al. 2000). Im Vergleich von herkömmlichen Computertomographen und der DVT kommt die CBCT-Technologie mit einer deutlich geringeren effektiven Strahlendosisbelastung für den Patienten aus (Ludlow und Ivanovic 2008, Chau und Fung 2009, Loubele et al. 2009, Suomalainen et al. 2009). Die verschiedenen DVT-Geräte gehen jedoch auch mit unterschiedlichen Dosis-Werten einher (Ludlow et al. 2006). Der NewTom 9000 geht mit einer effektiven Dosis von 56,5 $\mu \mathrm{Sv}$ (Brooks 2005) bzw. 6 mSv (Mozzo et al. 1998, Heurich et al. 2002) einher. Für den Accuitomo wurden Dosiswerte von 20,02 $\mu \mathrm{Sv}$ bis $43,27 \mu \mathrm{Sv}$ (Hirsch et al. 2008) bzw. $18 \mu \mathrm{Sv}$ bis $66 \mu \mathrm{Sv}$ (Okano et al. 2009) gezeigt. Spitzenwerte von 7,4 $\mu$ Sv konnten erreicht werden, womit das Accuitomo mit Panoramaschichtaufnahmen vergleichbar ist (Arai 2001). Im Vergleich von 3 DVTs (Accuitomo 3D, I-CAT, NewTom 3G) geht der Accuitomo 3D mit der geringsten und der I-CAT mit der höchsten effektiven Dosis für den Patienten einher (Loubele et al. 2009).

In einer Vergleichsstudie von CBCT und MSCT wurden effektive Dosiswerte für MSCT-Geräte von $685 \mu \mathrm{Sv}$ (ICRP 1990) und $1410 \mu \mathrm{Sv}$ (ICRP 2008) festgestellt, die damit höher sind als die der CBCT. Bei Verwendung von low-dose-MSCT hingegen sind beide Verfahren ähnlich. Aufgrund der relativ großen Streuung der Dosiswerte und der Bildqualität ist die Optimierung der Darstellungsparameter bei MSCTUntersuchungen, aber auch bei der CBCT, von enormer Wichtigkeit (Soumalainen et al. 2009). Die mittlere effektive Dosis der DVT liegt bei $221 \pm 275 \mu \mathrm{Sv}$, während die 
der CT bei $788 \pm 334 \mu$ Sv liegt (nach ICRP 103) (Valentin 2007, Ludlow und Ivanovic 2008, Loubele et al. 2009).

Der CTDI ist der allgemein akzeptierte technische Maßstab für die Dosis von CTSystemen, der für Einzelschichtaufnahmen der CT definiert worden ist (Shope et al. 1981). Mit der Einführung von Mehrschichtsystemen mit Kollimierungen von $20-160 \mathrm{~mm}$ in der MSCT und bis zu $200 \mathrm{~mm}$ in der Flachdetektor-CT ist die konventionelle CTDI-Definition nicht mehr adäquat (Dixon 2003, Kyriakou et al. 2008). Vergleiche von CT-Systemen, einschließlich ihrer Dosismerkmale, sind gegenwärtig immer noch schwierig. Ein sehr allgemeiner Ansatz sind Monte-CarloBerechnungen für phantom- oder patientenspezifische Dosisberechnungen, die sowohl für integrale Dosiswerte als auch für Dosisverteilungen zu nutzen sind (Kyriakou et al. 2008). Insgesamt gesehen sind die Dosiswerte bei typischen Einstellungen der fpVCT mit denen einer MSCT vergleichbar (Gupta et al. 2008). Anhand dieser Unterschiede wird die Bedeutung einer strengen klinischen Indikation für eine diagnostische Strahlenbelastung deutlich. Es konnte bereits gezeigt werden, dass sich der Einsatz einer Computertomographie aus strahlenhygienischen Gründen insbesondere auf komplexere traumatologische und onkologische Kasuistiken sowie präoperative Planungen bei Syndromen und der Nutzung von Navigationssystemen beschränkt. Zudem ist die CT zur Planung von Zahnimplantaten bei bereits vorhandenen DVT-Kapazitäten nicht mehr indiziert, da bereits DVT-Daten zur Implantationsplanung zur Anwendung kommen. Nachweislich ergeben sich Vorteile bezüglich der Strahlenexposition aber auch der Kosten (Fuhrmann et al. 2003).

\subsection{Fragestellung}

Mit dem Einsatz von Flachbettdetektoren bei Volumencomputertomographen erhofft man sich neben einer höheren Volumenabdeckung eine deutlich höhere Ortsauflösung und verringerte Metallartefakte im Vergleich zu herkömmlichen MSCT. Im Rahmen der vorliegenden präklinisch-experimentellen Studie sollte evaluiert werden, wie differenziert enossale Implantate und periimplantäre Strukturen mit der neuen fpVCT-Technologie zerstörungsfrei dreidimensional darstellbar sind. 
Anhand radiologischer Bilder, die mittels fpVCT, DVT, Zahnfilm und OPG generiert wurden, wurden Implantate mit iatrogen gesetzten Defekten, periimplantäre Strukturen, eingelagertes Fremdmaterial und iatrogen gesetzte Knochendefekte befundet. Die Exaktheit der Befundung wurde verglichen, ebenso wie die Darstellbarkeit dieser Befunde in Abhängigkeit von der Art und Lokalisation. 


\section{Material und Methoden}

\subsection{Versuchsdurchführung}

\subsection{1. Überblick über die Versuchsdurchführung}

In der vorliegenden Studie wurden die Daten von vier verschiedenen radiologischen Verfahren durch drei Untersucher evaluiert. Dazu wurden in Schweinekiefer inserierte enossale Implantate und deren umgebende Strukturen radiologisch dargestellt und die Resultate ausgewertet.

Zu Beginn der Versuchsdurchführung (Fließdiagramm siehe Abb. 1) wurden an insgesamt 14 verwendeten enossalen Implantaten (siehe Kap. 2.4.) iatrogene Defekte gesetzt. Dazu zählten Defekte in Form von Längs- und Querrillen in Windungstiefe der Implantate und in Form von lokalisierten Planierungen der Implantatoberfläche. Anschließend wurde an 14 halben Unterkiefern von Schlachttieren ein Implantatbett durch Pilotbohrungen und Erweiterungsbohrungen für die Insertion der Implantate vorbereitet (siehe Kap. 2.5.). Vor der eigentlichen Implantatinsertion erfolgten zudem die Präparation iatrogener Alveolardefekte und das Einbringen von verschiedenen Fremdmaterialien wie Metallkugeln, Guttapercha und B-TCP in die vorbereitete Kavität (siehe Kap. 2.6.). Im Anschluss daran wurden die Implantate inseriert.

Es folgte dann die radiologische Evaluation der Präparate mit Hilfe der Zahnfilmaufnahme, der Orthopantomographie, der Digitalen Volumentomographie und der flatpanel-Volumencomputertomographie (siehe Kap. 2.7.). Sowohl von der DVT- als auch von der fpVCT-Aufnahme war für die anschließende Befundung der Bilddaten zunächst eine entsprechende Bildbearbeitung notwendig (siehe Kap. 2.7.3.1. und 2.7.4.1.). Um die vier Verfahren miteinander vergleichen zu können wurden die gesamten Bilddaten durch 3 Untersucher anhand eines Fragebogens befundet (siehe Kap. 2.8.), die erhobenen Daten evaluiert und statistisch ausgewertet (siehe Kap. 2.9.). 


\subsubsection{Fließdiagramm der Versuchsdurchführung}

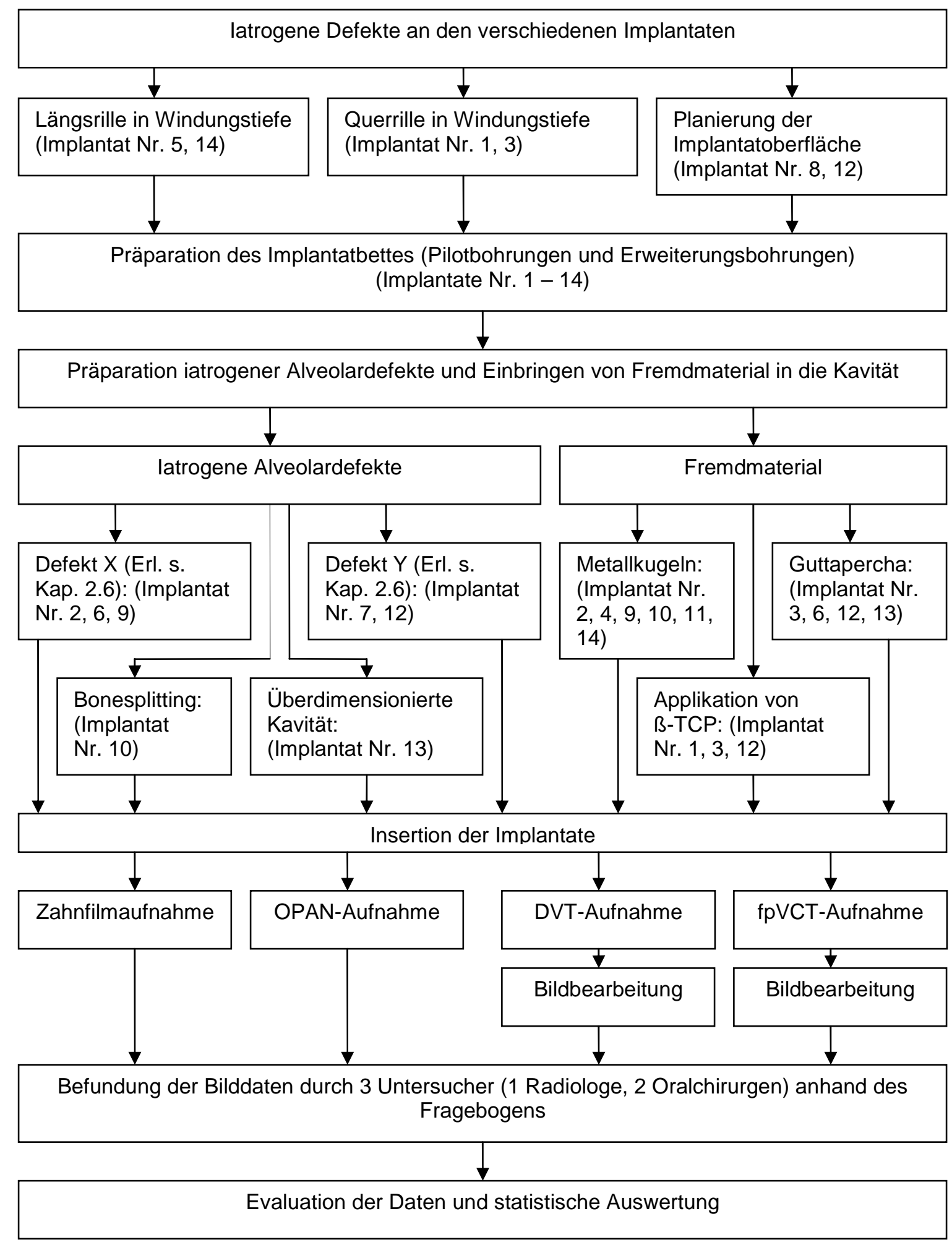

Abb. 1: Ablauf der Versuche 


\subsection{Materialliste}

Für die Implantation, die iatrogen gesetzten Implantat- und Alveolardefekte und die Fremdkörper wurden folgende Materialien verwendet:

- $\quad \beta$-TCP-Keramikpartikel der Körnung 500-1000 $\mu$ (Fa. Oraltronics, Bremen, Deutschland)

- chirurgische Pinzette (Aesculap AG, Tuttlingen, Deutschland)

- diamantiertes walzenförmiges Präparationsinstrument $(\varnothing 1 \mathrm{~mm}$, Länge 8 mm; Komet, Gebr. Brasseler, Lemgo, Deutschland)

- Digitaler Volumentomograph QR-DVT 9000 (Firma NEWTOM G3, Deutschland AG)

- Drehmomentschlüssel (Fa. Straumann, Basel, Schweiz)

- Edelstahlkugeln $(\varnothing 1 \mathrm{~mm}$ und 1,6 mm; Medizinische Werkstätten der Universitätsklinik, Göttingen, rostfreier Stahl)

- Einmal-Skalpell (Cutfix Fig. 22; B. Braun, Melsungen AG, Deutschland)

- Flatpanel-Volumencomputertomograph (General Electric Global Research Center, Niskayuna, NY, USA)

- Guttaperchastifte (0,3 mm dick, 1 mm lang; Dentalwelt, Köln, Deutschland)

- Implantate des Systems ITI- Bonefit (Fa. Straumann AG, Basel, Schweiz)

- Kamerastativ MA 190XPROB Pro (Manfrotto, Bogen Imaging GmbH, Köln, Deutschland)

- Konischer Präparationsdiamant $(\varnothing 1 \mathrm{~mm}$; Komet, Gebr. Brasseler, Lemgo, Deutschland)

- Kunststoff-Phantom (Georg-August-Universität Göttingen, Deutschland)

- Leitungswasser

- Lindemannfräse (Komet, Gebr. Brasseler, Lemgo, Deutschland)

- Manueller Gewindeschneider (FA. Straumann, Basel, Schweiz)

- Messlehre (Keydent Beerendonk, Seitz\&Herag, Linden, Deutschland)

- Nadelhalter nach Hegar- Mayo (DUROGRIP 150 mm gerade, Aesculap AG, Tuttlingen, Deutschland)

- Orthopantomographie Orthophos Plus Ceph (Sirona Dental Systems, Bensheim, Deutschland) 
- Plexiglasgestell (Georg-August-Universität Göttingen, Deutschland)

- Plexiglas-Röhre (Höhe: 22cm, Durchmesser: $12 \mathrm{~cm}$, Wanddicke: 0,5 cm, Baumarkt, Göttingen, Deutschland)

- Raspatorium (Raspatory/ Elevators, ERGOPLANT doppelendig, Aesculap AG, Tuttlingen, Deutschland)

- Röntgenfilm (Agfa Dentus M2 comfort, single film E/F size 2, Heraeus Kulzer, Hanau, Deutschland)

- Rosenbohrer (Komet, Gebr. Brasseler, Lemgo, Deutschland; Ø $3 \mathrm{~mm}$ )

- scharfer Löffel nach Hemingway (HEMINGWAY 170 mm Fig. 2, Aesculap AG, Tuttlingen, Deutschland)

- scharfe 15er Nadeln (Aesculap AG, Tuttlingen, Deutschland)

- Schlachttiere, halbe Unterkiefer vom Schwein (Harzer Fleisch Vermarktung, Schlachthof Göttingen, $\mathrm{GmbH}$, Deutschland)

- 3,0 Seidenfaden (Silkam® Kurzfäden, metric 2, USP 3/0, B. Braun Melsungen AG, Deutschland)

- Silaplast-Abformmaterial, catf FUTUR (Detax GmbH \& Co. KG, Ettlingen, Deutschland)

- Systemkomponenten des ITI- Bonefit Systems (Fa. Straumann, Basel, Schweiz)

- Wangenhalter nach Langenbeck (Aesculap AG, Tuttlingen, Deutschland)

- Zahnfilm Heliodent (Sirona Dental Systems, Bensheim, Deutschland). 


\subsection{Schweinekiefer-Modell}

In der vorliegenden experimentellen Studie wurde anhand von Tiermodellen die radiologische Visualisierung enossaler Implantate untersucht. Als Tiermodell dienten hierfür halbe Unterkiefer von Schlachttieren (Harzer Fleisch Vermarktung, Schlachthof Göttingen, $\mathrm{GmbH}$ ). Diese wurden vor der Untersuchung im Gefrierfach gelagert, so dass sie zu Versuchsbeginn in tief gefrorener Form vorlagen.

\subsection{Enossale Implantate}

Für die Insertion der enossalen Implantate lagen insgesamt 14 Implantate des Systems ITI-Bonefit der Institut Straumann AG (Basel, Schweiz) vor. Es handelte sich um Implantate mit der Bezeichnung Standard Implantat, regular neck, SLA, Material Titan, mit variablen Dimensionen bzgl. Länge $(8 \mathrm{~mm}, 10 \mathrm{~mm}, 12 \mathrm{~mm}, 14$ $\mathrm{mm}, 16 \mathrm{~mm}$ ) und Durchmesser (3,3 mm, 4,0 mm, 4,1 mm). Weitere Unterschiede neben Durchmesser und Länge waren außerdem die zusätzlich im Gewindebereich gesetzten Defekte. So wurden zwei der Implantate mit einer Querrille auf unterschiedlicher Höhe des Gewindebereiches und zwei der Implantate mit einer Längsrille entlang des gesamten Gewindebereiches versehen. An zwei weiteren Implantaten wurden je zwei Gewindegänge planiert (Tab. 2).

Die Implantate vom Durchmesser $3.3 \mathrm{~mm}$ und $4.1 \mathrm{~mm}$ waren Implantate der neueren derzeit handelsüblichen Generation der Firma Straumann. Die Implantate des Durchmessers $4.0 \mathrm{~mm}$ zählten zu einer älteren Implantatgeneration derselben Firma, die sich lediglich im Durchmesser, jedoch nicht hinsichtlich des Materials von der neueren Implantatgeneration unterschieden. 


\begin{tabular}{|c|c|c|}
\hline $\begin{array}{c}\text { Implantat- } \\
\text { Nr. }\end{array}$ & $\begin{array}{c}\text { Durchmesser }(\mathbf{m m}) / \\
\text { Länge }(\mathbf{m m})\end{array}$ & latrogen erzeugte Defekte am Implantat \\
\hline 1 & $4.0 / 14$ & untere 2 Implantatwindungen entfernt \\
\hline 2 & $3.3 / 16$ & obere Implantatwindung entfernt \\
\hline 3 & $4.1 / 10$ & -------- \\
\hline 4 & $4.0 / 16$ & 1 Längsrille in Windungstiefe \\
\hline 5 & $4.1 / 14$ & -------- \\
\hline 6 & $4.1 / 08$ & -------- \\
\hline 7 & $3.3 / 12$ & Implantatwindungen, 0,5 mm tief \\
\hline 8 & $4.0 / 14$ & ------- \\
\hline 9 & $4.1 / 10$ & -------- \\
\hline 10 & $4.1 / 10$ & -------- \\
\hline 11 & $4.1 / 10$ & 1 Querrille zwischen oberen 2 Implantatwindungen, \\
\hline 12 & $4.1 / 10$ & 0,5 mm tief \\
\hline 13 & $4.1 / 08$ & -------- \\
\hline 14 & $3.3 / 08$ & 1 Längsrille in Windungstiefe \\
\hline
\end{tabular}

Tab. 2: Größe und Merkmale der insgesamt 14 inserierten enossalen Implantate.

\subsubsection{Defekte an Implantaten}

Es wurden alle 14 vorliegenden Implantate in je einen Schweinekiefer inseriert. Um bei der anschließenden röntgenologischen Darstellung Unterschiede der einzelnen Implantate herausarbeiten und beurteilen zu können, unterschieden sich die 14 Implantate sowohl in Länge als auch im Durchmesser und hinsichtlich der iatrogenen Defekte (siehe Kapitel 2.4., Tab. 2, Abb. 2 A-C). 

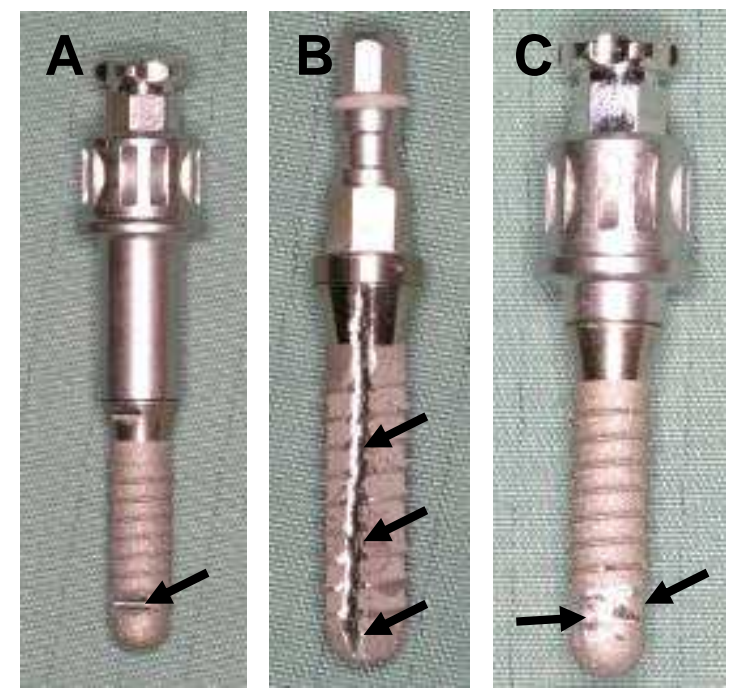

Abb. 2: Dentalimplantat mit iatrogenen Defekten: A Querrille im Windungsschaft, B Längsrille, $\boldsymbol{C}$ Entfernung der unteren 2 Windungen.

Die Längs- und Querrillen wurden mit Hilfe einer diamantierten Walze präpariert (Durchmesser: $1 \mathrm{~mm}$, Länge: $8 \mathrm{~mm}$ ). Dabei wurde die Walze bis zum größten Umfang ihres Durchmessers im Implantat versenkt, so dass eine Rille von 0,5 mm Tiefe entstand. In einer anschließenden Kontrolle, die mit Hilfe einer Messlehre erfolgte, konnte die Tiefe verifiziert werden. Die Längsrille wurde entlang des gesamten Implantatgewindes von Implantatspitze bis zum Implantathals gesetzt. Die Querrillen wurden auf zwei unterschiedlichen Höhen des Implantates wie folgt präpariert: zum einen zwischen den beiden oberen Windungen, zum anderen zwischen den beiden unteren Windungen des Implantatgewindes.

Ein weiterer Defekt wurde durch die Entfernung von Windungen mittels einer Lindemannfräse präpariert. Dabei wurden entweder die obere oder die unteren beiden Windungen am Implantatgewinde an einer Seite des Implantates planiert.

\subsection{Präparation des Implantatbettes und Insertion der Implantate}

Für die Insertion wurden die Schweinekiefer mit den Händen auf einer festen Unterlage fixiert. Mit Hilfe eines Wangenhalters nach Langenbeck wurden die Weichteile des Kieferabschnittes von dem zu präparierenden Kieferkamm abgehalten, um freien Zugang zur Zahnreihe zu erhalten. Die Präparation eines trapezförmigen Mukoperiostlappens erfolgte mit einem Einmal-Skalpell, chirurgischer Pinzette und Raspatorium in der unbezahnten Eckzahnregion der Schweinekiefer. 
Die Entlastungsschnitte erfolgten nach vestibulär und in kürzerem Ausmaß nach lingual. Der Ankörnung des Kieferkammes mit einem Rosenbohrer am Ort der geplanten Implantatinsertion folgten die Pilotbohrung und die Erweiterungsbohrungen mit den entsprechenden Systemkomponeneten des ITIBonefit Systems unter externer chirurgischer Absaugung (Abb. 3.1 A, B).
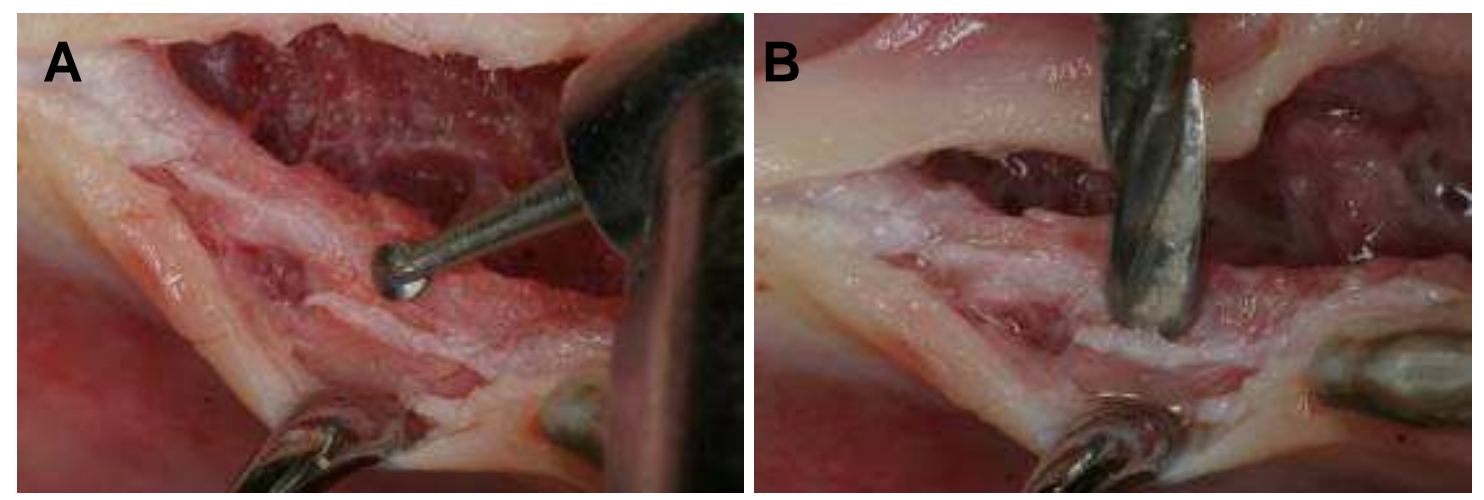

Abb. 3.1: Freipräparierter Schweinekieferkamm mit dem geplanten Insertionsort für das Implantat, A Ankörnung des Kieferkammes mit einem Rosenbohrer, B Pilotbohrung.

Danach wurde ein Gewinde in der Kavität für das Implantat mit einem manuellen Gewindeschneider präpariert, um anschließend das korrespondierende Implantat inserieren zu können.

Die Insertion der Implantate erfolgte mit Hilfe eines Drehmomentschlüssels unter Druck von koronal (Abb. 3.2 C-E).

Im Anschluss erfolgten die Reposition des Lappens und die Nahtversorgung mit einem Nadelhalter nach Hegar-Mayo, scharfen 15er Nadeln, chirurgischer Pinzette und 3,0 Seidenfaden (Abb. 3.2 F). 

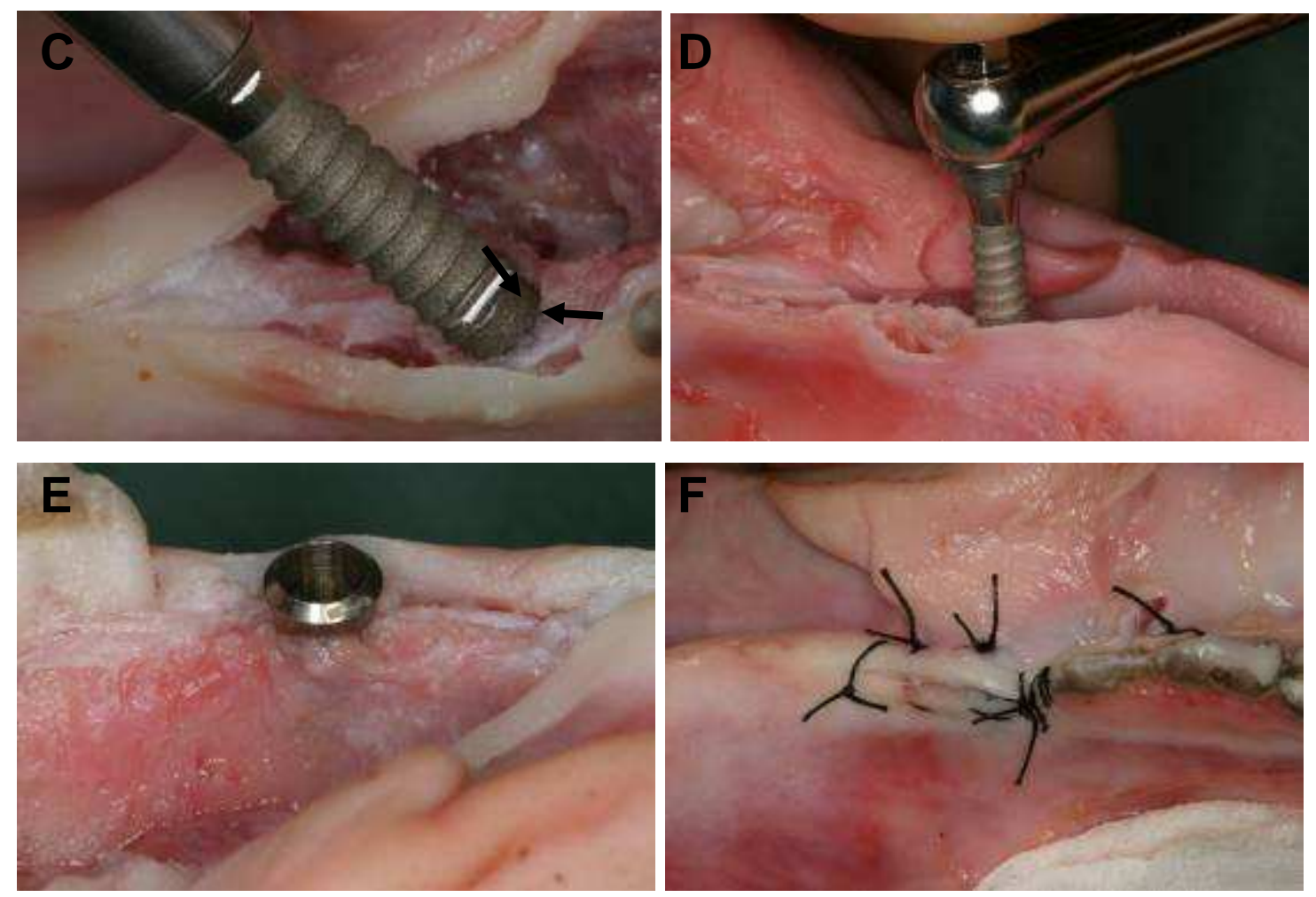

Abb. 3.2: Freipräparierter Schweinekieferkamm mit dem geplanten Insertionsort,

C Dentalimplantat an der vorbereiteten Implantatkavität, Beginn der Insertion (Pfeil: Querrille), D Implantatinsertion mit Drehmomentschlüssel und Druck von koronal, $\boldsymbol{E}$ Inseriertes Dentalimplantat im Alveolarkamm eines Schweinekiefers,

$\boldsymbol{F}$ Nahtversorgung nach Implantatinsertion und erfolgter Lappen-Reposition.

\subsection{Künstliche Defekte an Alveole und Positionierung von Fremdkörpern}

Bei zwölf der vierzehn Schweinekieferpräparate wurden vor der Implantatinsertion einerseits iatrogene Defekte an den Alveolen präpariert (8 Präparate) andererseits verschiedene Fremdmaterialien in das Implantatbett eingebracht (11 Präparate). Als intraalveoläre Defekte wurden zum einen mit einem konischen

Präparationsinstrument $1 \mathrm{~mm}$ tiefe keilförmige Bohrungen in $4 \mathrm{~mm}$ Höhe und zum anderen Längsrillen von $1 \mathrm{~mm}$ Tiefe und einer Länge $\leq 10 \mathrm{~mm}$ mit einem walzenförmigen Schleifer in die Wand des Implantatbettes präpariert (Lokalisation siehe Tab. 3). Als Fremdkörper wurden Edelstahlkugeln mit einem Durchmesser von $1 \mathrm{~mm}$ und 1,6 mm verwendet. Als weitere Fremdkörper fanden 0,3 mm dicke und 1 mm lange Guttaperchastifte und $\beta$-TCP-Keramikpartikel der Körnung 500-1000 m Verwendung (Abb. 4 A-C). 
Die genaue Zuordnung der Implantatdefekte, Alveolardefekte und der Fremdkörper zu den einzelnen Schweinekieferproben ist Tabelle 3 zu entnehmen.

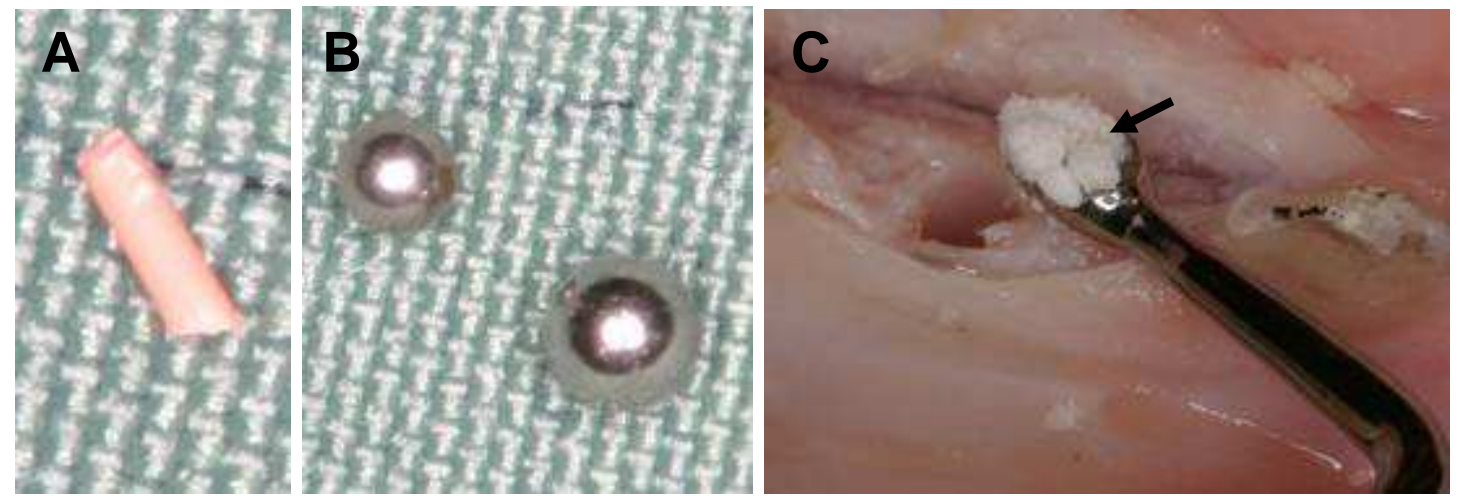

Abb. 4: A Guttapercha- Stift, $\boldsymbol{B}$ Metallkugeln mit $\varnothing 1 \mathrm{~mm}$ und $\varnothing 1,6 \mathrm{~mm}$ (Medizinische Werkstätten der Universitätsklinik, Göttingen), C $\beta$-TCP-Keramikpartikel der Körnung 500-1000 $\mu m$ (Fa. Oraltronics, Bremen, auf Hemmingway-Löffel). 


\begin{tabular}{|c|c|c|c|c|}
\hline $\begin{array}{l}\text { Implantat- } \\
\text { Nr. }\end{array}$ & $\begin{array}{c}\text { Durchmesser (D)/ } \\
\text { Länge (L) } \\
\text { der Implantate } \\
\text { (Angabe in mm) }\end{array}$ & $\begin{array}{l}\text { Implantatdefekt } \\
\text { (Art, Lokalisation) }\end{array}$ & $\begin{array}{l}\text { Fremdkörper } \\
\text { (Anzahl, Art) }\end{array}$ & $\begin{array}{c}\text { Alveolardefekt } \\
\text { (Art, Lokalisation) }\end{array}$ \\
\hline 1 & D: 4,0 / L:14 & $\begin{array}{l}\mathrm{B} \text {, untere } 2 \\
\text { Windungen planiert }\end{array}$ & B-TCP & --------- \\
\hline 2 & $\mathrm{D}: 3,3 / \mathrm{L}: 16$ & - ----- & 1x Stahlkugel Ø1 mm & A, mesial \\
\hline 3 & $\mathrm{D}: 4,1 / \mathrm{L}: 10$ & $\begin{array}{l}\text { B, obere Windung } \\
\text { planiert }\end{array}$ & $\begin{array}{l}\text { B-TCP und } \\
\text { 1x Guttapercha 30er Point, } \\
\text { Länge je } 1 \mathrm{~mm}\end{array}$ & -------- \\
\hline 4 & $\mathrm{D}: 4,0 / \mathrm{L}: 16$ & --------- & $1 \times$ Stahlkugel $\varnothing 1 \mathrm{~mm}$ & -------- \\
\hline 5 & $\mathrm{D}: 4,1$ / L: 14 & $\begin{array}{l}\text { A, distal des } \\
\text { Alveolarkammes }\end{array}$ & -------- & --------- \\
\hline 6 & D: 4,1 / L: 8 & -------- & $\begin{array}{l}\text { 1x Guttapercha 30er Point, } \\
\text { Länge je } 1 \mathrm{~mm}\end{array}$ & $A$, lingual \\
\hline 7 & D: 3,3 / L: 12 & --------- & --------- & $\begin{array}{l}\mathrm{B} \text {, vestibulär } 0,5 \mathrm{~mm} \\
\text { tief }\end{array}$ \\
\hline 8 & D: 4,0 / L: 14 & $\begin{array}{l}\text { C, zwischen unteren } \\
2 \text { Windungen }\end{array}$ & --------- & --------- \\
\hline 9 & $\mathrm{D}: 4,1 / \mathrm{L}: 10$ & --------' & 3x Stahlkugel $\varnothing 1 \mathrm{~mm}$ & $\begin{array}{l}\text { A, vestibulär; } \\
\text { Perforation in Höhe } 4 \\
\text { mm }\end{array}$ \\
\hline 10 & D: 4,1 / L: 10 & --------- & $\begin{array}{l}\text { 1x Stahlkugel } \varnothing 1 \mathrm{~mm} \text { und } \\
1 \times \text { Stahlkugel } \varnothing 1,6 \mathrm{~mm}\end{array}$ & Bonesplitting \\
\hline 11 & D: 4,1 / L: 10 & -------- & 1x Stahlkugel $\varnothing 1,6 \mathrm{~mm}$ & --------- \\
\hline 12 & $\mathrm{D}: 4,1 / \mathrm{L}: 10$ & $\begin{array}{l}\text { C, zwischen oberen } 2 \\
\text { Windungen }\end{array}$ & $\begin{array}{l}\text { B-TCP und } \\
\text { 1x Guttapercha 30er Point, } \\
\text { Länge je } 1 \mathrm{~mm}\end{array}$ & $\begin{array}{l}\text { B, distal } \\
0,5 \mathrm{~mm} \text { tief }\end{array}$ \\
\hline 13 & D: 4,1 / L: 8 & -------- & $\begin{array}{l}\text { 2x Guttapercha 30er Point, } \\
\text { Länge je } 1 \mathrm{~mm}\end{array}$ & Überdimension, L:12 \\
\hline 14 & D: 3,3 / L: 8 & $\begin{array}{l}\text { A, vestibulär des } \\
\text { Alveolarkammes }\end{array}$ & $\begin{array}{l}\text { 1x Stahlkugel } \varnothing 1 \mathrm{~mm} \text { und } \\
1 \times \text { Stahlkugel } \varnothing 1,6 \mathrm{~mm}\end{array}$ & $\begin{array}{l}\text { Ersatz, lingual } \\
\text { perforiert }\end{array}$ \\
\hline
\end{tabular}

Tab. 3: Zuordnung der Implantatdefekte, Alveolardefekte und Fremdkörper zu den einzelnen Schweinekieferproben; siehe Legende.

\section{Legende:}

- Implantatdefekte: A: Längsrille in Windungstiefe, B: 1-2 Windungen entfernt, C: Querrille $0,5 \mathrm{~mm}$ tief

- Fremdkörper: $\beta$ - TCP-Keramik, Körnung 500 - 1000 um; portioniert mit 2 kleinen Löffeln nach Hemmingway

- Alveolardefekte: X: $1 \mathrm{~mm}$ tiefe, keilförmige Bohrung in $4 \mathrm{~mm}$ Höhe

Y: Längsrille von $1 \mathrm{~mm}$ Tiefe und $\leq 10 \mathrm{~mm}$ Länge (Diamantwalze);

Tiefendimension

- Zusätzliche Alveolardefekte:

1. Perforation in Höhe $4 \mathrm{~mm}$ : Perforation des Alveolarkammes durch Insertion des Implantates, in $4 \mathrm{~mm}$ Höhe von koronal ausgehend

2.Bonesplitting: künstliche Spaltung des Alveolarkammes bei Implantatinsertion

3.Überdimension, L:12: vorgebohrte Kavität im Verhältnis zum Implantat zu groß, Kavitättiefe entspricht einer Implantatlänge von $12 \mathrm{~mm}$

4. Ersatz, lingual perforiert: Perforation bei Implantatinsertion eines im Verhältnis zur Kavität zu großen Implantates, anschließend Insertion eines kleineren Implantats bei bleibender Perforation, lingual 


\subsection{Röntgenologische Verfahren}

\subsubsection{Zahnfilmaufnahmen}

In dieser Studie wurden Zahnfilmaufnahmen der Präparate angefertigt. Die Aufnahmen erfolgten mit dem Gerät Heliodent (Sirona Dental Systems, Bensheim, Deutschland (technische Daten siehe Tab. 4)).

Der Zahnfilm bezeichnet die Einzelaufnahme bestimmter Zähne in Form einer intraoralen Aufnahme. Der Röntgenfilm hat hierbei eine Standardgröße von $5 \times 4 \mathrm{~cm}$ und befindet sich während der Aufnahme hinter dem darzustellenden Zahn. Er liegt damit als letztes Objekt im Strahlengang. Die Röntgenquelle liegt außerhalb des Mundes. Dabei tritt der Zentralstrahl der Röntgenröhre senkrecht auf eine Tangente des Zahnbogens in der Kauebene in der Höhe des zu untersuchenden Zahnes (orthoradiale Projektion). Aufnahmebedingt können mit dieser Technik etwa 2 - 4 Zähne auf einem Röntgenfilm dargestellt werden. Zahnfilmaufnahmen ermöglichen eine präzise zweidimensionale Darstellung der anatomischen Beziehungen zu Nachbarzähnen, Knochenstrukturen und benachbarten Nerven sowie der Zähne selbst.

Ziel der Zahnfilmaufnahme ist die möglichst isometrische Abbildung des Zahnes auf dem Röntgenfilm und eine regelrechte Darstellung im Bezug zu den Nachbarzähnen. Technische Daten des Heliodent, Sirona Dental Systems, Bensheim, Deutschland siehe Tab. 4).

\begin{tabular}{|l|c|}
\hline Technische Daten & $60 \mathrm{kV}$ \\
\hline Röhrenspannung & $7 \mathrm{~mA}$ \\
\hline Röhrenstromstärke & $0,12 \mathrm{sec}$ \\
\hline Aufnahmezeit & $1: 30$ \\
\hline Puls-Pausen-Verhältnis & $2 \mathrm{~mm} \mathrm{AL}$ \\
\hline Filterung & $0,7 \mathrm{~mm}$ \\
\hline Brennfleckgröße & \\
\hline
\end{tabular}

Tab. 4: Technische Daten des Heliodent (Sirona Dental Systems, Bensheim, Deutschland). 
Für die Zahnfilmaufnahme jedes einzelnen Schweinekieferblockes wurden die 14 Präparate zunächst einzeln so auf einer planen Unterlage in waagerechter Position auf dem Zahnfilm platziert, dass Röntgenfilm und Zahnreihe parallel zueinander lagen. In einem Abstand von $2 \mathrm{~cm}$ und in rechtem Winkel zur Zahnreihe wurde der Zentralstrahl der Röntgenröhre dann auf die zu untersuchende Zahnregion des Schweinkiefers ausgerichtet (Abb. 5), um eine orthoradiale Projektion zu erzielen. Die Aufnahme erfolgte in Rechtwinkeltechnik mit Strahlenbegrenzung. Folgende Aufnahmeparameter lagen vor: Röhrenspannung 60 kV, Röhrenstromstärke $7 \mathrm{~mA}$, Aufnahmezeit 0,12 sec. Eine nachfolgende Bildbearbeitung war nicht erforderlich.

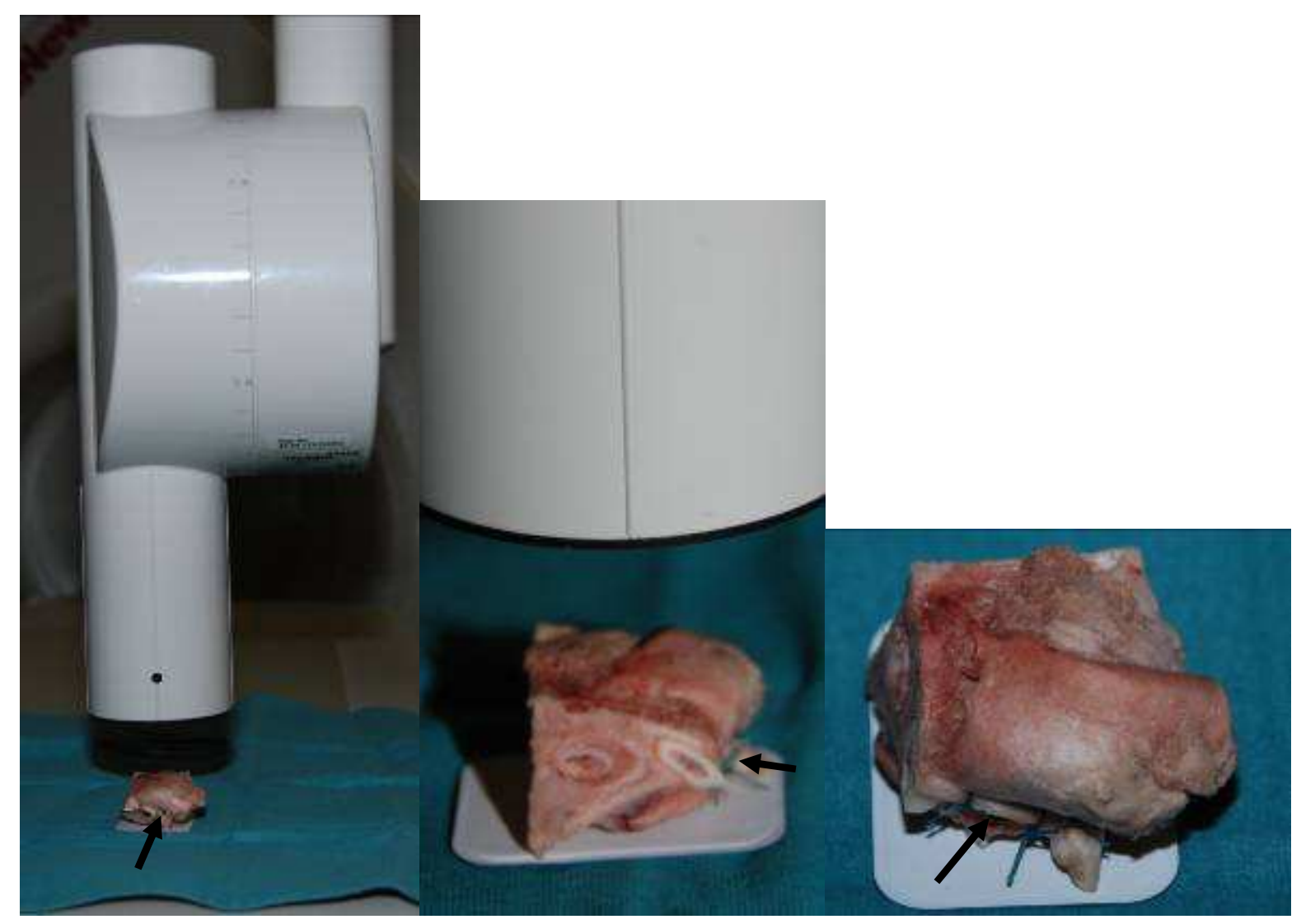

Abb. 5: Positionierung der Schweinekiefersegmente auf der Unterlage (Pfeil: Zahnimplantat). 


\subsubsection{Orthopantomographie (OPAN)}

Die OPAN-Aufnahmen der Implantate wurden mit dem Gerät Orthophos Plus Ceph (Sirona Dental Systems, Bensheim, Deutschland) angefertigt.

Das Aufnahmegerät besteht aus einer Aufnahmeeinheit und einer Röntgenröhre. Die Aufnahmeeinheit fährt mit einer Filmkassette, von links kommend, um den Hinterkopf des Patienten herum und beschreibt somit etwas mehr als einen Halbkreis. Synchron hierzu umfährt die Röntgenröhre den Kopf des Patienten von rechts nach links. Die Röntgenröhre emittiert hierbei ein keilförmiges, senkrechtes Strahlenbündel, das sich von 0,25 mm auf ca. $3 \mathrm{~mm}$ erweitert. Dieses Strahlenbündel durchleuchtet die Kieferabschnitte und passiert dann eine Schlitzblende aus Blei. Die sich dahinter befindende Kassette bewegt sich in Fahrtrichtung etwas schneller als die gesamte Apparatur, so dass der Film entsprechend belichtet werden kann. Technische Daten des Gerätes sind Tab. 5 zu entnehmen.

Ziel dieses Aufnahmeverfahrens ist eine Übersicht zum Erkennen pathologischer Prozesse im Mund-Kiefer-Bereich, ohne dabei jedoch genaue Details einzelner Zähne und Strukturen darstellen zu können.

\begin{tabular}{|l|c|}
\hline Technische Daten & \\
\hline Röhrenspannung & $60-90 \mathrm{kV}$ (60 kV) \\
\hline Röhrenstromstärke & $9-16 \mathrm{~mA} \mathrm{(14} \mathrm{mA)}$ \\
\hline Leistungsaufnahme & $2,8 \mathrm{~kW}$ \\
\hline Gesamtfilter & $2,5 \mathrm{~mm} \mathrm{AL}$ \\
\hline Brennfleckgröße & $0,5 \times 0,5 \mathrm{~mm}$ \\
\hline Umlaufzeiten Panorama & $19 \mathrm{sec}-108 \mathrm{sec}$ \\
\hline Belichtungszeiten Panorama & $4,9 \mathrm{sec}-25,3 \mathrm{sec}$ \\
\hline Schaltzeiten Ceph Film & $0,1 \mathrm{sec}-4,0 \mathrm{sec}(17 \mathrm{Stufen})$ \\
\hline
\end{tabular}

Tab. 5: Technische Daten des Orthophos Plus/ CD/ Plus Ceph (Sirona Dental Systems, Bensheim, Deutschland); im Rahmen der Studie angewandte Parameter fett gedruckt.

Um die 14 Schweinekiefer für die Orthopantomographie entsprechend des Gerätes genau ausrichten zu können, wurde jeder einzelne Schweinekieferblock mit Hilfe von 
Silaplast-Abformmaterial zunächst in ein Kunststoff-Phantom eingesetzt, das anatomisch etwa einem ausgewachsenen menschlichen Unterkiefer entspricht. Dieses Kunststoff-Phantom wurde anschließend mit Hilfe von zwei Schrauben in ein Plexiglasgestell eingespannt, das für experimentelle OPAN-Aufnahmen angefertigt wurde. Dieses Gestell konnte mit einem Schraubgewinde auf einem Kamerastativ in die benötigte Position des Orthopantomographen gebracht werden. Im Folgenden wurde der Phantom-Unterkiefer mit dem darauf befestigten Schweinkieferblock in der Kinnstütze des Gerätes positioniert (Abb. 6 A-C). Die Aufnahmeparameter sind Tabelle 5 (fett gedruckt) zu entnehmen.
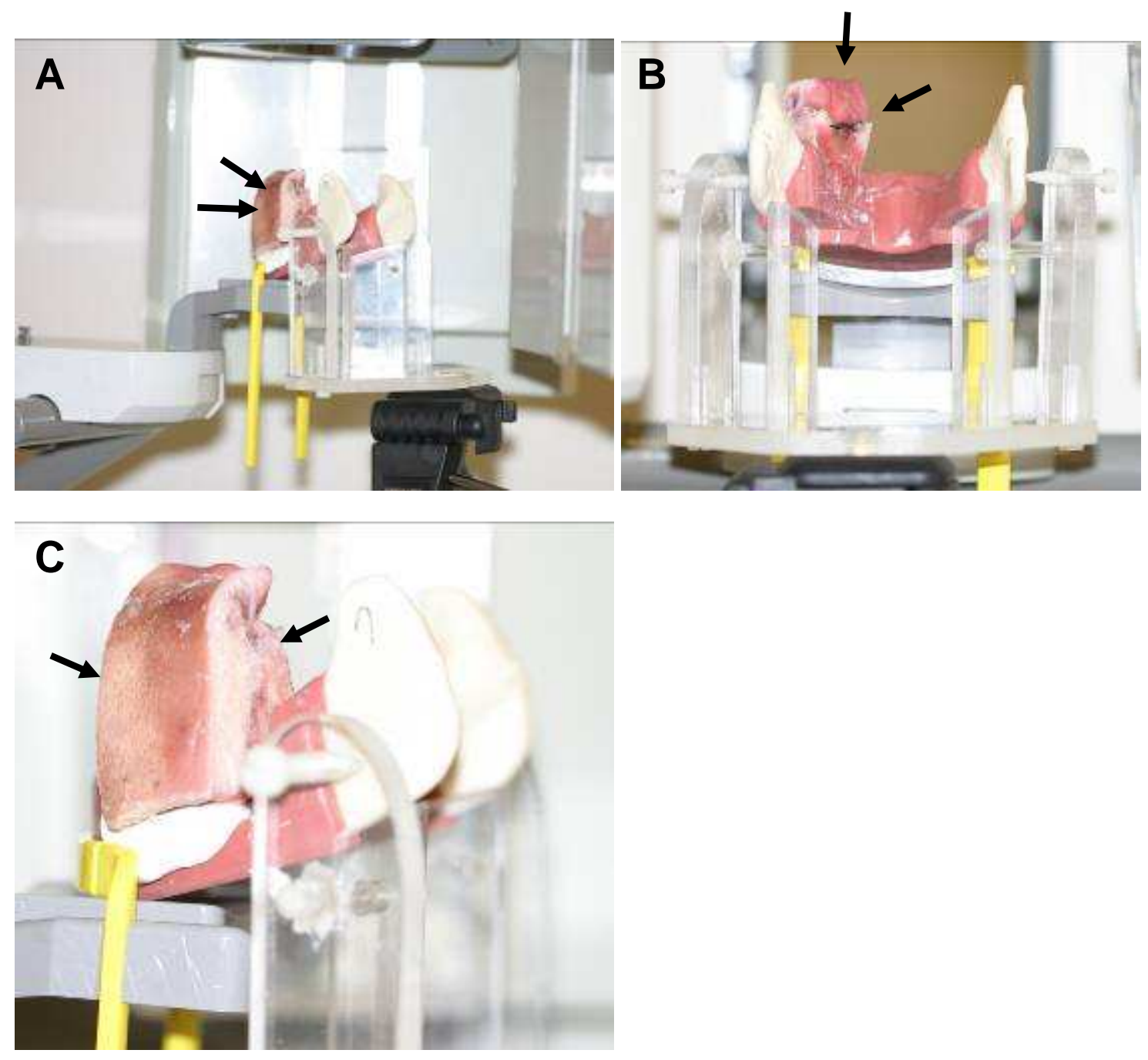

Abb. 6: A Schweinekieferblock in Unterkiefer-Kunststoff-Phantom, B Phantom in Plexiglasgestell, $\boldsymbol{C}$ Positionierung des Schweinekieferblockes. (Pfeil:

Schweinekieferblock). 


\subsubsection{Digitale Volumentomographie (DVT)}

Die DVT ist ein dreidimensionales bildgebendes Verfahren. Das hier angewandte Gerät war das Modell QR-DVT 9000 (Firma NEWTOM G3, Deutschland AG). Der Digitale Volumentomograph (DVT) besitzt neben einer um $360^{\circ}$ rotierbaren Röntgenröhre einen Detektor als Bildverstärker und CCD-Chips für die Bilderfassung. Bei der Bilderstellung rotieren Röntgenröhre und Bildverstärker um die fixierte Patientenliege. Dabei wird pro Grad ein Einzelschnitt erstellt.

Aus allen 360 Bilddaten wird anschließend der Rohdatensatz errechnet. Aus den Axialschichten der primären Rekonstruktion werden, je nach Bedarf, weitere sekundäre Rekonstruktionen errechnet.

Technische Daten des Gerätes finden sich in Tabelle 6.

Ziel der DVT-Methode ist die Darstellung von Hochkontrastunterschieden und die freie Wahl einer Ebene. Neben Übersichtsdarstellungen des Mund-KieferBereiches sind hier zusätzlich Detaildarstellungen von Knochen, Dentin, Schmelz, Parodontalspalt usw. möglich.

\begin{tabular}{|l|l|}
\hline Aufnahmeverfahren & \\
\hline Aufnahmeverfahren & Cone beam computed tomography \\
\hline Aufnahmebereich & Kugelvolumen mit konusförmigem Strahlenverlauf \\
\hline Aufnahmemodus & $\begin{array}{l}1 \times 360^{\circ} \text { um den unbewegten Patienten } \\
360 \text { Einzelaufnahmen - 1 Aufnahme pro Grad der } \\
\text { Umdrehung }\end{array}$ \\
\hline Größe des Aufnahmefeldes & $150 \mathrm{~mm}$ Durchmesser \\
\hline Aufnahmedauer & $36,0 \mathrm{~s}$ \\
\hline Filterung & $12,8 \mathrm{~mm}$ Aluminium \\
\hline Brennfleckgröße & variabel 0,5 oder 1,5 automatisch gewählt \\
\hline Röntgenstrahler & \\
\hline Röhrentyp & Hochfrequenzröhre mit automatischer Belichtung \\
\hline Röhrenspannung & $110 \mathrm{kV}$ \\
\hline Röhrenstromstärke & variabel 0,5 - 1,5 mA automatisch während der \\
& Aufnahme geregelt \\
\hline
\end{tabular}

Tab. 6: Technische Daten des Modell QR-DVT 9000 (Firma NEWTOM G3, Deutschland AG). 
Für die Aufnahme der DVT-Daten wurden die Schweinekieferblöcke zunächst in einer Plexiglas-Röhre mit $22 \mathrm{~cm}$ Höhe, $12 \mathrm{~cm}$ Durchmesser und einer Wanddicke von $0,5 \mathrm{~cm}$ mit Silaplast-Abformsilikon mittig im Inneren der Röhre befestigt. Dabei lag die Zahnreihe waagerecht auf dem Boden der Röhre. Anschließend wurde die Plexiglasröhre mit Leitungswasser randvoll gefüllt. Die gefüllte Röhre wurde nun auf der Untersuchungsliege des Digitalen Volumentomographen positioniert und so ausgerichtet, dass die gewünschte Anatomie des Schweinekieferblockes gerade in der Gantry lag (Abb. 7). Die Aufnahmeparameter sind Tabelle 7 zu entnehmen.

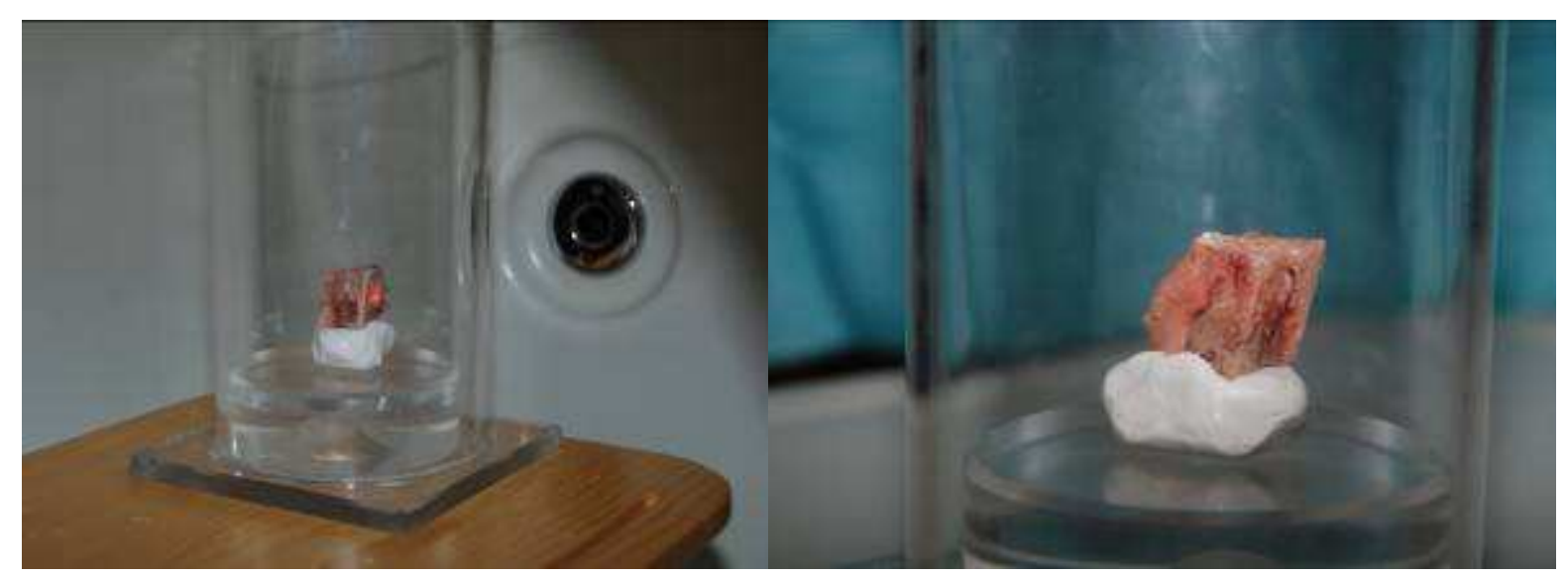

Abb. 7: Positionierung des Schweinekieferblockes in der Plexiglasröhre, Positionierung im DVT (zu fotografischen Zwecken Darstellung ohne Leitungswasser).

\begin{tabular}{|l|l|}
\hline Technische Daten & \\
\hline Röhrenspannung & $110 \mathrm{kV}$ \\
\hline Röhrenstromstärke & $\begin{array}{l}\text { variabel } 0,5-1,5 \mathrm{~mA} \text { automatisch während der } \\
\text { Aufnahme geregelt }\end{array}$ \\
\hline Projektionen / Rotation & $360^{\circ}$ \\
\hline Größe des Aufnahmefeldes & $150 \mathrm{~mm}$ Durchmesser \\
\hline Rotationszeit & $36,0 \mathrm{~s}$ \\
\hline Filterung & $12,8 \mathrm{~mm}$ Aluminium \\
\hline Brennfleckgröße & variabel 0,5 oder 1,5 automatisch gewählt \\
\hline Rekonstruktionsparameter (Auflösung) & $0,15 \mathrm{~mm}$ \\
\hline Matrixgröße (Bildpunkte) & $512 \times 512$ Bildpunkte axial \\
\hline
\end{tabular}

Tab. 7: angewandte Aufnahmeparameter DVT. 
Während sich die Röntgenröhren-Detektoreinheit in einer einzelnen $360^{\circ}$ Drehung um die Plexiglasröhre drehte, erfolgte die Aufnahme der radiologischen Projektionen. Alle digital durch den Detektor aufgenommen Projektionen bildeten den

Rohdatensatz, der auf der Workstation Windows XP Professional abgelegt wurde. Anschließend wurde der Rohdatensatz im Rahmen einer Bildrekonstruktion zur Auswertung aufbereitet. Aus den gewonnen Rohdatensätzen konnte ein Volumendatensatz errechnet werden, aus dem sich die unterschiedlichen für die Auswertung notwendigen Projektionsrichtungen ermitteln ließen.

\subsubsection{Bildbearbeitung DVT}

Da insbesondere eine dreidimensionale Darstellung für die Beurteilung der Daten erforderlich war, kam die Professional Version der DVT 9000 Software zum Einsatz. Diese Software ermöglicht neben der dreidimensionalen Darstellung des Objektes auch das Messen von Winkeln und Strecken, Graustufenveränderungen, 3DMarkierungen und Profilmessungen.

\subsection{4. flatpanel-Volumencomputertomographie (fpVCT)}

Das hier angewandte fpVCT ist einer von weltweit drei Prototypen des General Electric (GE) Global Research Centers (Niskayuna, NY, USA) und dient ausschließlich Forschungszwecken an der Universität Göttingen.

Die fpVCT ist eine neuartige CT-Bildgebungstechnik, die eine gesteigerte isotrope Ortsauflösung von $200 \mu \mathrm{m}$ (10\% MTF) ermöglicht. Sie beinhaltet eine normale CT-Gantry, die einen Röntgenstrahler, Performix 630 (GE Medical Systems, NY, USA) und zwei Flächendetektoren besitzt (Abb. 8). 

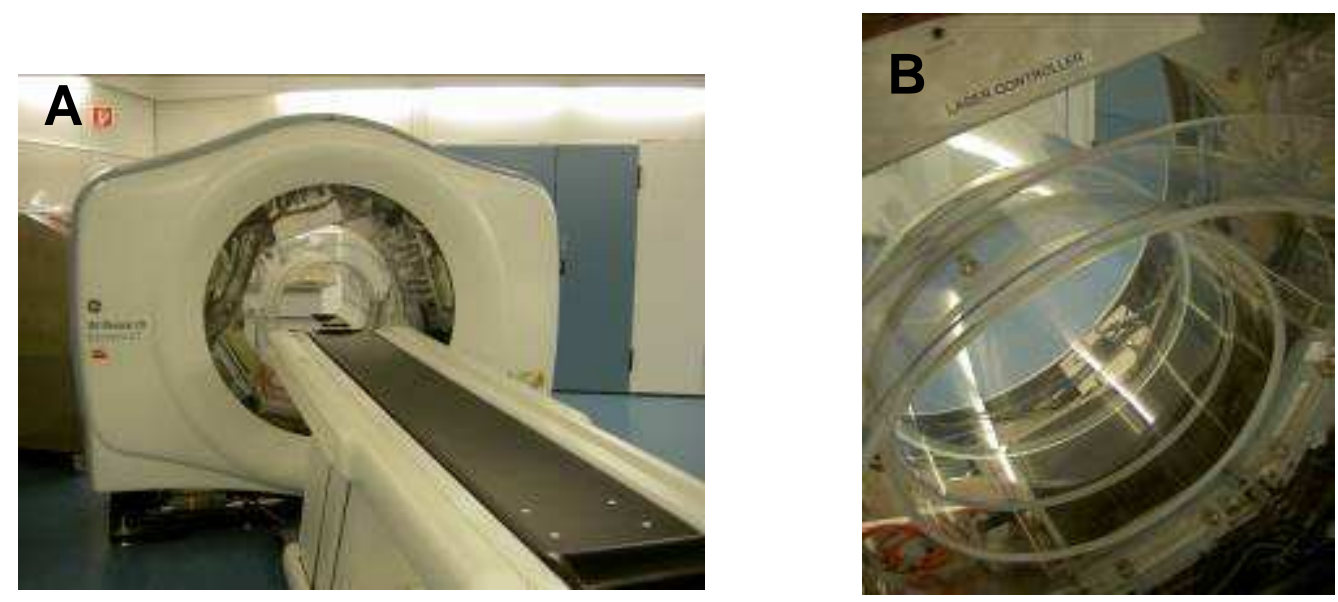

Abb. 8: A fpVCT-Prototyp (GE Research Volumetric CT, GE Global Research Center, Niskayuna, NY, USA), B Detailansicht Gantry.

Die Flachbettdetektoren führen mit dem Röntgenstrahler eine gekoppelte Drehbewegung von $360^{\circ}$ um ein gemeinsames Rotationsz entrum durch. Die quadratischen Detektoren sind 20,5 × 20,5 cm² groß und enthalten $1024 \times 1024$ Detektorelemente. Ein Detektorelement hat dabei eine Größe von $200 \times 200 \mu m^{2}$ und besteht aus amorphem Silizium (a-Si) mit einer Szintillationsschicht aus Thallium dotierten Cäsiumjodid (Csl:TI) (Abb. 9). Von den 1024 Elementen können orthogonal zur Systemachse optional bis maximal 360 Zeilen genutzt werden. Beide Flächendetektoren stoßen in einem Winkel von ca. $120^{\circ}$ parallel zur Systemachse aneinander. Die Detektoren ermöglichen, dass das System wahlweise je nach Größe des Versuchsobjektes sowohl im Ein- als auch im Zweidetektorbetrieb genutzt werden kann (technische Daten der Flachbettdetektoren siehe Tab. 8).

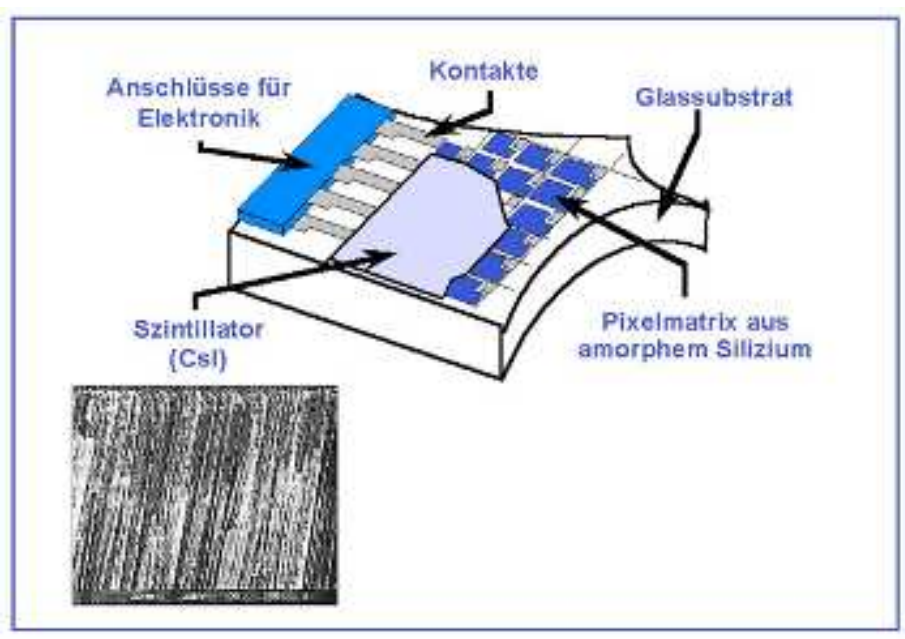

Abb. 9: Detektortechnik des verwendeten fpVCT (Guderjahn T 2005) 


\begin{tabular}{|l|c|}
\hline Flachbettdetektoren & \\
\hline Aktive Fläche & $20,5 \mathrm{~cm} \times 20,5 \mathrm{~cm}$ \\
\hline Kantenlänge jedes einzelnen Detektorelements & $200 \mu \mathrm{m}$ \\
\hline Anzahl der Detektorelemente der Sensormatrix & $1024 \times 1024$ \\
\hline Nutzung der Detektorelemente parallel zur Systemachse & 1024 Spalten \\
\hline Detektorelemente senkrecht zur Systemachse & 120,180 oder 360 Zeilen \\
\hline
\end{tabular}

Tab. 8: Technische Daten der Flachbettdetektoren.

Als Röntgenquelle kam eine luftgekühlte GE Performix 630-Röntgenröhre mit Wolframanode zum Einsatz. Sie war auf eine Röhrenleistung von $20 \mathrm{~kW}$ begrenzt und gab Strahlung in Form eines Kegelstrahles mit einem Öffnungswinkel von $17^{\circ}$ auf die Flächendetektoren ab.

Die Öffnungsfläche der Gantry hatte einen Durchmesser von $43,8 \mathrm{~cm}$. Das axiale Field of View (FOV) betrug beim Einsatz des Ein-Detektor-Betriebs 12,8 cm $\times 12,8$ $\mathrm{cm}$ und im Zwei-Detektor-Betrieb 33,3 cm × 33,3 cm (Technische Daten des Röntgenstrahlers und des Strahlenganges siehe Tab. 9).

Für die 3D Rekonstruktion wurde der Feldkampalgorithmus verwendet. Zur endgültigen Auswertung und Bearbeitung der Bilder diente eine mit Bildbearbeitungsund 3D-Rendering-Software ausgestattete Windows Advantage Workstation Version AW4.2.06.02.EXT der Firma General Electric (GE Medical Systems, NY, USA). 


\begin{tabular}{|c|c|}
\hline Röntgenstrahler & \\
\hline Fokusgröße & $0,7 \mathrm{~mm}$ \\
\hline Max. Röhrenspannung & $140 \mathrm{kV}$ \\
\hline Röhrenstromstärke & bis $400 \mathrm{~mA}$ \\
\hline Röhrenleistung & $20 \mathrm{~kW}$ \\
\hline \multicolumn{2}{|l|}{ Strahlengang } \\
\hline Abstand Röhre vom Rotationszentrum & $54,0 \mathrm{~cm}$ \\
\hline Fokus- Detektor- Abstand bei Ein- Detektor- Betrieb & $78,3 \mathrm{~cm}$ \\
\hline Fokus- Detektor- Abstand bei Zwei- Detektor- Betrieb & $80,3 \mathrm{~cm}$ \\
\hline Gantry- Öffnung & $43,8 \mathrm{~cm}$ \\
\hline Bildausschnitt (Field of View) bei Ein- Detektor- Betrieb & $12,8 \mathrm{~cm} \times 12,8 \mathrm{~cm}$ \\
\hline Bildausschnitt (Field of View) bei Zwei- Detektor- Betrieb & $33,3 \mathrm{~cm} \times 33,3 \mathrm{~cm}$ \\
\hline $\begin{array}{l}\text { z- Achsenabschnitt/ Rotation bei } 360 \text { Detektorzeilen bei Ein- Detektor- } \\
\text { Betrieb }\end{array}$ & $42,0 \mathrm{~mm}$ \\
\hline $\begin{array}{l}\text { z- Achsenabschnitt/ Rotation bei } 360 \text { Detektorzeilen bei Zwei- } \\
\text { Detektor- Betrieb }\end{array}$ & $33,8 \mathrm{~mm}$ \\
\hline Maximale Objektlänge innerhalb eines Scan- Vorgangs & $210 \mathrm{~mm}$ \\
\hline Vergrößerungsfaktor (Isozentrum) & 1,43 \\
\hline Auflösung (10\% MTF) & $200 \mu \mathrm{m}$ \\
\hline
\end{tabular}

Tab. 9: Technische Daten des Röntgenstrahlers Performix 630 (GE Medical Systems, NY, USA).

Für die Akquisition der fpVCT-Daten wurden die geviertelten Schweinekiefer mit den eingesetzten Zahnimplantaten einzeln auf der Untersuchungsliege des fpVCT in der Systemachse positioniert. Um den folgenden Scan dem Material und der Größe der einzelnen Präparate anpassen zu können, wurden vorher die einzelnen Parameter entsprechend ausgewählt. Der Scan jedes einzelnen Präparates konnte in einer Schicht im Ein-Detektorbetrieb erfolgen. Die Akquisitionsparameter sind Tab. $10 \mathrm{zu}$ entnehmen. 


\begin{tabular}{|l|c|}
\hline Technische Daten & $80 \mathrm{kV}$ \\
\hline Röhrenspannung & $100 \mathrm{~mA}$ \\
\hline Röhrenstromstärke & 1000 \\
\hline Projektionen / Rotation & 360 \\
\hline Zeilen & $8 \mathrm{~s}$ \\
\hline Rotationszeit & 125 \\
\hline Projektionen / Sekunde & $800 \mathrm{mAs} /$ Rotation \\
\hline Röhrenstrom-Zeit-Produkt / Rotation & $0,09 \mathrm{~mm}$ \\
\hline Rekonstruktionsparameter (Auflösung) & $512 \times 512$ \\
\hline Matrixgröße (Bildpunkte) & \\
\hline
\end{tabular}

Tab. 10: Akquisitionsparameter fpVCT.

Anschließend erfolgte die Bildrekonstruktion. Da die aus einer Kegelstrahlgeometrie resultierende Bildrekonstruktion sehr rechenintensiv ist und die fpVCT umfangreiche Projektionsdatenmengen erzeugt (Hermann et al. 2005), war eine parallele Datenverarbeitung erforderlich. Aus den gewonnen Rohdatensätzen konnte dann ein Volumendatensatz errechnet werden, aus dem sich die einzelnen für die Auswertung der Daten notwendigen Projektionsrichtungen ermitteln ließen.

\subsubsection{Bildbearbeitung fpVCT}

Die gewonnen Bilddatensätze konnten nun an die Windows Advantage Workstation 4.2, GE Medical Systems (Workstation) zur weiteren Bearbeitung übertragen werden. Mit der Volume Rendering Technik (VRT) war die Bearbeitung der 3D-Darstellungen möglich. Es wurden drei verschiedene Darstellungsprotokolle erstellt, um die Zahnimplantate in den Schweinekiefern anhand verschiedener Aspekte besser beurteilen zu können. Es wurden adäquate Farben, Lichtdurchlässigkeit und Kontrast entsprechend der Houndsfield Einheit (HE) für die Darstellung der verschiedenen Gewebe und Zahnimplantate definiert. Die Protokolle lassen sich wie folgt beschreiben: 
- Transparentmodus I: Dieses Protokoll diente insbesondere der Darstellung von metallischem Material. Eine Substanz mit einer Dichte entsprechend des Implantates ( 3000 HE) wurde rot gefärbt, dentinartiges Gewebe gelb und restliches umliegendes Knochen- und Weichgewebe wurde in Schwarz-WeißKontrast belassen. In diesem Modus war eine Durchsicht durch umliegendes Gewebe auf die Zähne und das Implantat möglich, so dass das Implantat in Relation dazu einschließlich Fremdkörper und Defekt beurteilt werden konnte.

- Transparentmodus II: Dieses Protokoll diente vor allem in Verbindung mit dem „Front-Cut-Modus“ (s.u.) der Messung von Länge und Durchmesser der Implantate.

- Spezielles Zahnprotokoll: Diese Form der Darstellung diente vor allem der überlagerungsfreien Darstellung verschiedener Zahnstrukturen wie Zahnschmelz, Dentin und Pulpa und eignete sich gut, um diese Strukturen gegeneinander abzugrenzen.

In jedem dieser drei Protokolle bestand des Weiteren die Möglichkeit, eine Darstellung sowohl im „No-Cut-Modus“ als auch im „Front-Cut-Modus“ durchzuführen (siehe Abb. 10). Während der „No-Cut-Modus“ die Betrachtung des Präparates von der Aufsicht im Rahmen einer gesamten Rotation zulässt, bietet der „Front-CutModus" die Möglichkeit, einen virtuellen Schnitt durch den Schweinekiefer durchzuführen und so die Kante zwischen Implantat und Kiefer besser betrachten zu können.
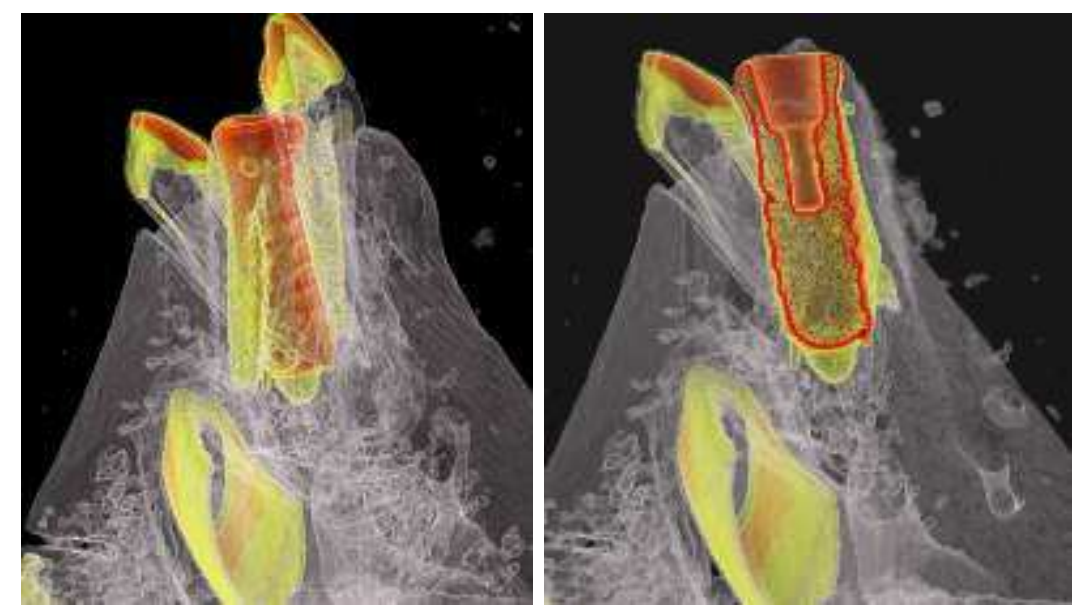

Abb. 10: fpVCT; VR-Technik, Transparentmodus I; $\boldsymbol{A}$ "No-Cut-Modus", B "Front-CutModus" 


\subsection{Geblindete Evaluation der Bilddatensätze durch drei Untersucher}

Es erfolgte der Vergleich zwischen den vier radiologischen Verfahren. Dabei wurden neben den konventionellen dentalen Röntgenverfahren Zahnfilm und Orthopantomographie auch die Digitale Volumentomographie und die neuartige Flächendetektor-Volumencomputertomographie-Methode im Rahmen einer randomisierten geblindeten Studie evaluiert.

\subsubsection{Untersucher}

Alle vorliegenden Bilddaten wurden von drei Ärzten ausgewertet. Zwei der Auswertenden waren zahnärztliche Chirurgen und Implantologen mit Schwerpunkt zahnärztliche Radiologie. Der dritte Auswertende war ein Facharzt für Radiologie ohne spezielle Erfahrung in zahnärztlicher Radiologie und ohne besondere zahnmedizinische Kenntnisse.

\subsubsection{Kalibrierung der Untersucher}

Da alle Untersucher bisher keine praktische Erfahrung mit dem fpVCT hatten, wurden Protokolle zur Auswertung vorgegeben und damit eine standardisierte Einarbeitung in die dazugehörige Workstation vor der eigentlichen Befundung der Daten durchgeführt. Den Untersuchern wurde dabei gezeigt, mit welchen Protokollen und in welcher Vorgehensweise sowohl die enossalen Implantate mit ihren Längenund Durchmesserangaben und verschiedenen Defekten als auch die verschiedenen Alveolardefekte und Fremdmaterialien am besten dargestellt und beurteilt werden konnten. Die Protokolle enthielten dabei Angaben über das schrittweise Vorgehen an der Workstation zum Erreichen der einzelnen Einstellungen für die Beurteilung der Daten. 
Zudem wurden allen Untersuchern vor Beginn der Datenbefundung mögliche Zielgrößen angegeben. So waren neben den möglichen Durchmesserangaben der Implantate auch die möglichen Längenangaben vorher bekannt. Ebenso wurden die Untersucher über die Art der möglichen Fremdmaterialien und möglichen Implantatund Alveolardefekte informiert.

\subsubsection{Prüfbogen}

Für die Analyse der einzelnen Bilddaten ist ein Fragebogen erstellt worden (siehe Abb. 11). Dieser umfasste zunächst die Frage nach Erkennen des Implantates und dessen Länge und Durchmesser. Zu beurteilen war weiterhin die Differenzierbarkeit von Spongiosa und Kompakta. Bezüglich der Alveole war die Frage nach möglichen Defekten, deren Art, Ausmaß, Lokalisation und inrer dreidimensionaler Darstellung zu beantworten. Hinsichtlich des Zahnimplantates war ebenfalls die Frage nach Defekten und deren Art, Ausmaß, Lokalisation und dreidimensionaler Darstellung zu beantworten. Zudem wurde nach den zugeführten Fremdkörpern gefragt, deren Art, Anzahl, Größe und Lokalisation. Eine Beurteilung bezüglich möglicher Streustrahlenartefakte/ Aufhärtungsartefakte und die evtl. dadurch eingeschränkte Beurteilbarkeit der Bilddaten stellte ein weiteres Untersuchungskriterium dar. Mögliche weitere Befunde konnten ebenfalls mit aufgeführt werden. 


\section{Auswertungsbogen Implantate im Schweinekiefer \\ Geblindete Begutachtung der Präparate}

Präparat Nr.:

Untersucher:

Verfahren (bitte ankreuzen)

$\begin{array}{llll}\text { OPAN } & \text { fpVCT } & \text { DVT } & \text { Zahnfilm }\end{array}$

Implantat vorhanden? - ja - nein

Länge (ohne Tulpe):

Durchmesser (äußere Windungen):

Differenzierung Spongiosa/ Kompakta/ Zahnschmelz/ Dentin möglich: - ja - nein

Knochendefekte am Implantat? - ja - nein

Dreidimensionale Darstellung des Knochendefektes möglich? - ja - nein

Art der Defekte:

Dimension des Defektes in $\mathrm{mm}$ :

Lokalisation des Defektes (in Relation zum Implantat):

Defekte am Implantat? - ja - nein

Art der Defekte:

Dimension der Defekte in $\mathrm{mm}$ :

Lokalisation der Defekte:

Dreidimensionale Darstellung der Implantatdefekte? - ja - nein

Fremdkörper im Interface- Bereich?

Art und Anzahl?

Lokalisation:

Größe:

Streustrahlenartefakte/ Aufhärtungsartefakte?

Beeinträchtigung der Bildauswertung durch Artefakte? - ja - nein

Weitere Befunde?

Abb. 11: Prüfbogen 


\subsubsection{Blindung und Randomisierung der Datensätze}

Mit jedem der vier Bildgebungsverfahren wurden alle vierzehn Schweinekieferproben mit den Implantaten, Defekten und Fremdkörpern dargestellt. Um einer möglichen Wiedererkennung bei der Datenbefundung anhand der Kombination der zugeführten Fremdkörper oder Defekte zu den verschieden großen Implantaten zu umgehen, wurde mittels Zufallszahlen eine einfache Randomisierung (Schumacher und Schulgen 2002) durchgeführt (Medizinische Statistik der Universität Göttingen). Anschließend befundeten alle drei Untersucher unabhängig voneinander in gleicher Abfolge die einzelnen Bilddaten der vier Darstellungsverfahren (Reihenfolge siehe Kap. 2.8.5.). Die Befundung wurde geblindet durchgeführt. Für alle Auswertenden lagen gleiche Untersuchungsbedingungen vor.

\subsubsection{Durchführung der Evaluation}

Die Evaluation der Daten wurde an vier unterschiedlichen Tagen von jedem Untersucher einzeln durchgeführt. Jeder Untersucher erhielt dazu pro Tag 14 Bilddatensätze in randomisierter Reihenfolge. Dabei lagen den Untersuchern die Bilddatensätze in gleicher Reihenfolge vor. Die Bilddaten wurden in folgender Reihenfolge befundet (siehe Abb. 12): 


\begin{tabular}{|c|c|c|c|c|c|c|c|}
\hline 1 & Zahnfilm 3 & 15 & fpVCT 9 & 29 & fpVCT 1 & 43 & DVT 6 \\
\hline 2 & DVT 9 & 16 & OPAN 12 & 30 & fpVCT 12 & 44 & Zahnfilm 14 \\
\hline 3 & DVT 5 & 17 & fpVCT 11 & 31 & Zahnfilm 4 & 45 & Zahnfilm 9 \\
\hline 4 & OPAN 4 & 18 & OPAN 1 & 32 & DVT 8 & 46 & OPAN 13 \\
\hline 5 & DVT 13 & 19 & Zahnfilm 12 & 33 & DVT 1 & 47 & OPAN 8 \\
\hline 6 & Zahnfilm 8 & 20 & OPAN 10 & 34 & DVT 4 & 48 & DVT 10 \\
\hline 7 & fpVCT 3 & 21 & Zahnfilm 7 & 35 & DVT 11 & 49 & Zahnfilm 1 \\
\hline 8 & OPAN 5 & 22 & Zahnfilm 2 & 36 & DVT 3 & 50 & fpVCT 2 \\
\hline 9 & OPAN 7 & 23 & DVT 14 & 37 & Zahnfilm 11 & 51 & fpVCT 5 \\
\hline 10 & Zahnfilm 5 & 24 & DVT 12 & 38 & Zahnfilm 10 & 52 & fpVCT 4 \\
\hline 11 & Zahnfilm 13 & 25 & fpVCT 10 & 39 & OPAN 14 & 53 & OPAN 3 \\
\hline 12 & fpVCT 13 & 26 & fpVCT 8 & 40 & Zahnfilm 6 & 54 & fpVCT 14 \\
\hline 13 & OPAN 2 & 27 & fpVCT 7 & 41 & OPAN 11 & 55 & DVT 7 \\
\hline 14 & OPAN 9 & 28 & fpVCT 6 & 42 & OPAN 6 & 56 & DVT 2 \\
\hline
\end{tabular}

Abb. 12: Randomisierung der Datensätze: Reihenfolge der Zufallszahlen (einfache Randomisierung; Schumacher und Schulgen 2002)

\subsection{Statistische Auswertung}

Zum Vergleich der vier Röntgenmethoden wurde bei jeder Schweinekieferprobe gezählt, von wie vielen Untersuchern sowohl die Länge als auch der Durchmesser, das Vorhandensein von Knochen- bzw. Implantatdefekten oder das Vorliegen von Fremdkörpern korrekt festgestellt wurde. Anschließend wurde die Anzahl der korrekt erhobenen Befunde prozentual visualisiert.

Es wurde mit einem F-Test in einer nichtparametrischen Varianzanalyse (Prozedur „PROC GLM“ in der Statistik-Software SAS 9.1) weiterhin getestet, ob dieser Unterschied signifikant war. Das Signifikanzniveau lag bei $p<0,05$.

Des Weiteren wurde die Verteilung der einzelnen Messungen evaluiert und in Form von Boxplots dargestellt. Hierbei ließen sich nur unvollständige Boxplots aufführen, da die Messabweichungen in Relation zur Anzahl der zu beobachteten Größen zu gering waren.

Zudem erfolgte ein Vergleich zwischen zwei- und dreidimensionalen Verfahren und eine differenzierte Analyse der Fremdkörperaufdeckung. 
Abschließend wurde mit Hilfe des oben erwähnten statistischen Verfahrens der Einfluss des Untersuchers (1 Radiologe, 2 Oralchirurgen) auf die Qualität der Evaluation statistisch untersucht. 


\section{Ergebnisse}

\subsection{Bildbeispiele zur Darstellbarkeit von Implantaten und periimplantären}

Strukturen mit verschiedenen radiologischen Verfahren

Eine Evaluation der Präparate war mit allen untersuchten Verfahren möglich.

Bei der Betrachtung der Bilddaten durch die Untersucher konnten bei Anwendung aller vier radiologischen Verfahren Aussagen über die Nachbarschaftsverhältnisse umliegender Strukturen des Zahnimplantates getroffen werden. Mit allen vier Verfahren waren sowohl Zahnschmelz und Dentin als auch Substantia spongiosa und Substantia compacta klar differenzierbar. Ebenso waren Aussagen über das Vorhandensein von Zahnanlagen möglich. Die Zahnwurzeln und der Parodontalspalt waren ebenso beurteilbar (Beispiele Abb. 13 - 15).

Bei den Zahnfilm- und OPAN-Aufnahmen waren diese Aussagen auf den zweidimensionalen Raum beschränkt, so dass überlagerte Strukturen eingeschränkt beurteilt werden konnten (Beispiel Abb. 13 A - D). So wurde zum Beispiel das Vorliegen der zwei Metallkugeln im OPAN bei Präparat 10 als eine einzelne Kugel beschrieben (Abb. 13 C +D). Die Messung der Größe war dabei möglich, die Unterscheidung der zwei Kugeln hingegen nicht. Die Guttaperchastifte im Zahnfilm in Präparat 13 waren ebenso nur eingeschränkt beurteilbar. Der Stift kaudal des Implantates konnte korrekt beurteilt werden während der zweite Stift, der im Strahlengang hinter dem Implantatende lokalisiert war, lediglich zu etwa einem Drittel sichtbar war und in Folge dessen hinsichtlich seiner Größe nicht korrekt befundet werden konnte.

In beiden Verfahren konnten die Dentalimplantate in Bezug auf Größe, Lage und iatrogene Defekte untersucht werden. Die Lage des Defektes im Strahlengang hatte maßgeblichen Einfluss auf die Beurteilbarkeit. Das Implantatgewinde und der Implantatkopf waren deutlich zu erkennen, eine differenzierte Analyse einzelner Windungen war hingegen eingeschränkt, die Einsicht von kranial in den Implantatkopf hinein war nicht möglich. Im Vergleich war in der zweidimensionalen Darstellung die Qualität des Zahnfilms schärfer als die der OPAN.

Die Befundung einzelner Schichten des Implantatinneren oder der inneren Knochenstrukturen war ebenfalls nicht möglich. Verglichen mit den 
dreidimensionalen Verfahren konnte das Implantat lediglich aus der seitlichen Perspektive als Summationsabbildung beurteilt werden.
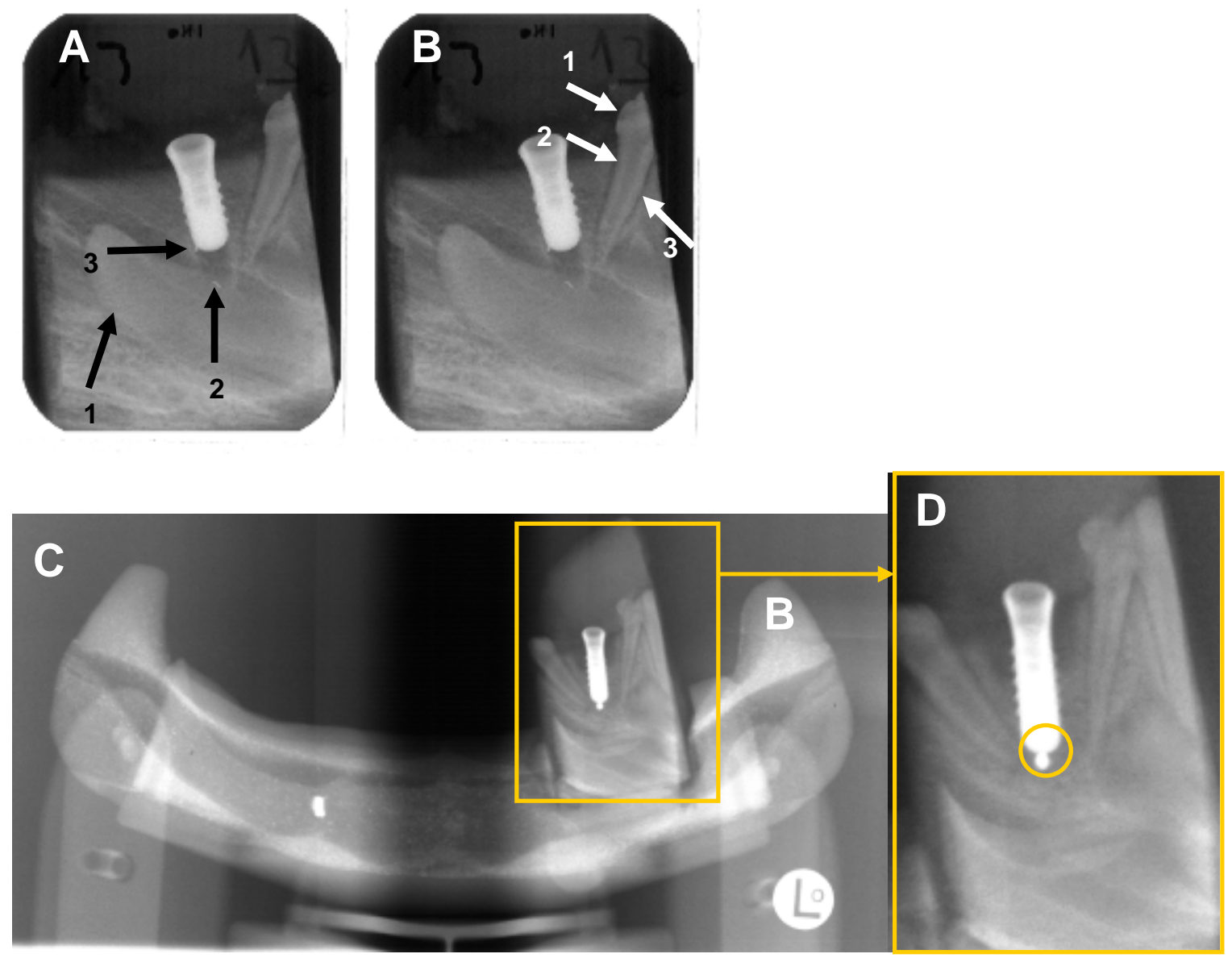

Abb. 13: A: Zahnfilmaufnahme (Präparat Nr. 13) Pfeil 1: Zahnanlage, Pfeil 2+3: Guttaperchastift, B: Zahnfilmaufnahme (Präparat Nr. 13) Pfeil 1: Zahnschmelz, Pfeil 2: Dentin, Pfeil 3: Parodontalspalt, C: OPAN-Aufnahme (Präparat Nr. 10) Übersichtsaufnahme: Schweinekieferblock (gelber Kasten) im Kunststoffphantom, D: OPAN-Aufnahme (Präparat Nr. 10) Detailaufnahme: Abbildung Schweinekieferblock, Kreis: eine von zwei Metallkugeln mit unterschiedlicher Größe erkennbar.

Mit den dreidimensionalen Aufnahmen von DVT und fpVCT konnten differenzierte Beschreibungen über die räumliche Zuordnung der umliegenden Gewebe und Fremdmaterialien zum Implantat gemacht werden (Abb. 14, 15.1 und 15.2). Die Aussagen über die Position einzelner Strukturen zueinander wie die im Alveolarknochen liegenden Zähne, tief im Knochen liegende Zahnanlagen und deren räumlicher Bezug zum Implantat oder auch die Fremdmaterialien waren insbesondere in der fpVCT wesentlich genauer als in Zahnfilm und OPAN. Auch 
verglichen mit der DVT waren präzisere Angaben durch die fpVCT möglich, was vor allem durch die VR-Technik und den Transparentmodus I gewährleistet wurde (Abb. 15.1 und 15.2).

Sowohl von den DVT-Daten als auch von den fpVCT-Daten ließen sich Volumendatensätze als 3D-Rekonstruktionen berechnen und bildlich darstellen (Abb. 14 B, C; 15.1 C, D; 15.2 E-H). Mit diesen Rekonstruktionen konnte das Objekt dreidimensional allseitig beurteilt werden. Auch die innen liegenden Schichten des Implantates oder der Knochen- und Zahnsubstanz konnten beurteilt werden. Insbesondere bei der fpVCT konnten dadurch einzelne Strukturen mit hoher Detailgenauigkeit und hoher Bildschärfe bei einer Ortsauflösung von $200 \mu \mathrm{m}$ (bei 10\% MTF) befundet werden (Abb. 15.1 und 15.2). Anhand der verschiedenen Darstellungsalgorithmen der fpVCT konnten die einzelnen Strukturen der Zahnsubstanz (Dentin, Zahnschmelz, Pulpa, Parodontalspalt), des Knochens (Substantia spongiosa und Substantia compacta) und die Implantate je nach Fragestellung somit präzise evaluiert werden.

Die minimal auftretenden Streustrahlenartefakte führten dabei zu unwesentlichen Beeinträchtigungen der Bildqualität. Auch die Oberflächenbeschaffenheit von Zahnsubstanz, Implantat und Gingiva ließen sich mit einer hohen Detailschärfe darstellen (Abb. 15.1 C+D).

Im Gegensatz zur fpVCT war die Auflösung der DVT deutlich geringer und die Bildqualität bei der 3D-Rekonstruktion wesentlich niedriger (Abb. 14). Die in den einzelnen Schichten der DVT dargestellten Strukturen von Knochen- und Zahnsubstanz sowie der eingelagerten Fremdmaterialien stellten sich unschärfer und damit schlechter beurteilbar dar als in der fpVCT (Abb. 14 A). Zudem erschienen in Abbildung $14 \mathrm{~B}+\mathrm{C}$ die Strukturen und Oberflächenbeschaffenheit der Gingiva und der Zahnkrone deutlich unebener als bei der fpVCT in Abbildung 15.1 C-D. Der Implantatkopf ließ sich gut vom umliegenden Gewebe abgrenzen, im Gegensatz zur fpVCT jedoch weniger präzise darstellen. Auch führten bei der DVT Streustrahlenartefakte vermehrt zu Beeinträchtigungen der Bildqualität und damit zu Einschränkungen in der Qualität der Datenerhebung. 


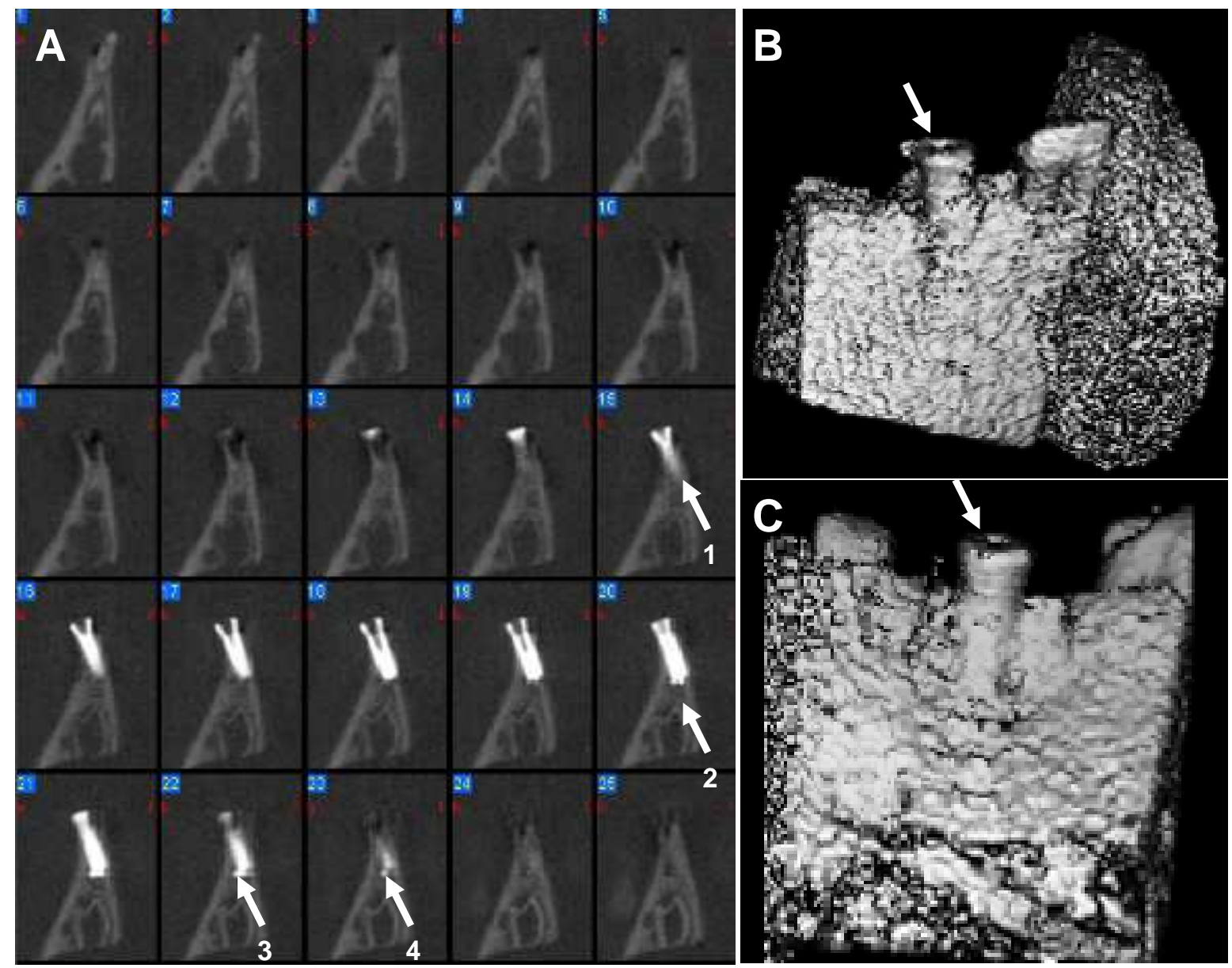

Abb. 14: DVT-Aufnahmen: A: Präparat Nr. 10, Pfeil 1: unsaubere Darstellung der Implantatspitze mit Streustrahlenartefakten, Pfeile 2-4: räumliche Zuordnung von zwei Metallkugeln zum Implantat, B: Präparat Nr. 8 Pfeil: Implantattulpe, C: Präparat Nr. 10 Pfeil: Implantatkopf.

In der fpVCT konnten die Konturen der einzelnen Strukturen sowohl von Alveolarknochen und Zahn als auch von Fremdmaterial scharf abgegrenzt werden. In allen Abbildungen war die präzise Beurteilung von Zahnschmelz, Dentin, Pulpa und dem Parodontalspalt möglich (Abb. 15.1 und 15.2). Zudem konnten exakte Aussagen hinsichtlich des Implantatkopfes getroffen werden. Dieser war bei allen Präparaten deutlich aus allen Richtungen einsehbar, so dass auch der Einblick von kranial in den Implantatkopf möglich war (Abb. 15.1 C-D). 

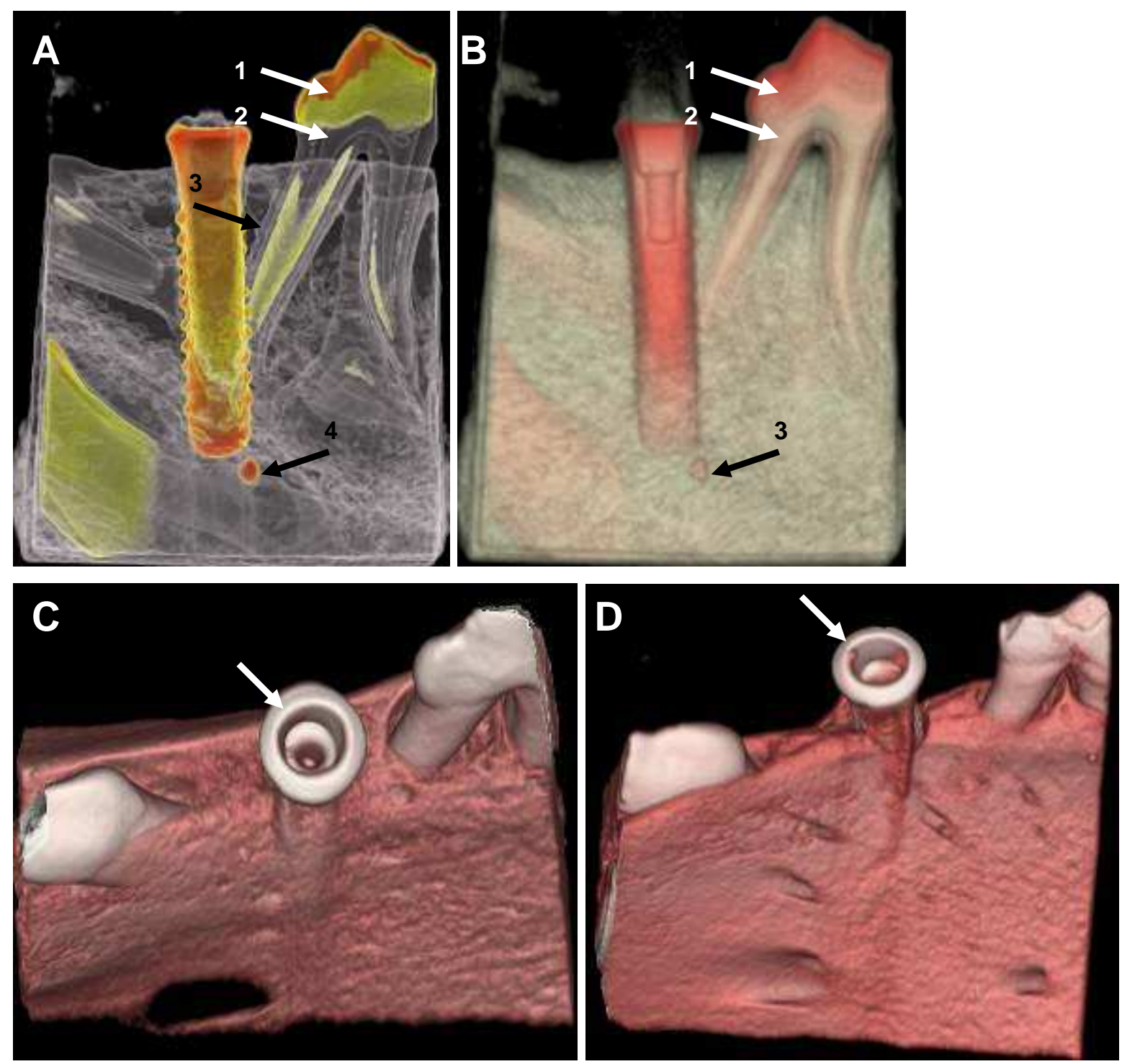

Abb. 15.1: Exemplarische Darstellung typischer Bilder der fpVCT (VR-Technik Volumendatensatz): A: Transparentmodus I (Pfeil 1: Dentin, Pfeil 2: Pulpa, Pfeil 3: Parodontalspalt, Pfeil 4: Metallkugel), B: Transparentmodus II (Pfeil 1: Dentin, Pfeil 2: Pulpa, Pfeil 3: Metallkugel), C+D: Ansicht von kranial (Pfeil: Implantattulpe eines inserierten Dentalimplantates), C: leicht verzerrte Darstellung des Implantates (oval)

In den folgenden Abbildungen sind deutlich die räumlichen Beziehungen der verschiedenen Implantate zu den umliegenden Zähnen, den ossären Strukturen und der Gingiva visualisiert. In dem Präparat der Abbildung 15.2 E war zum Beispiel der Kontakt der Implantatspitze mit dem tief im Alveolarknochen liegenden Zahnanteil evaluierbar. Außerdem ist deutlich die Lage der zwei Zähne im Knochen und der umgebenden Gingiva zu erkennen. Zudem war bei Präparat 15 (Abb. 15.2 F) die Perforation des Implantates nach Insertion zu beobachten. Es konnte deutlich das Heraustreten des Implantatkörpers auf der lingualen Seite des Alveolarknochens 
befundet werden. Der nach Perforation noch verbleibende Schleimhautüberzug war gut von dem Implantat abgrenzbar und konnte exakt beurteilt werden.

Zudem war mit der fpVCT eine genaue Einsicht in die inneren Strukturen des Implantates möglich (Abb. 15.2 G). Die einzelnen Schichten ließen sich präzise darstellen. Somit konnten die Relationen des Implantates zu einer Metallkugel und die räumliche Implantat-Knochen-Beziehung exakt beurteilt werden (Abb. 15.2 G - I). Ebenso waren eindeutige Aussagen hinsichtlich einzelner Anteile der Zahnsubstanz möglich.
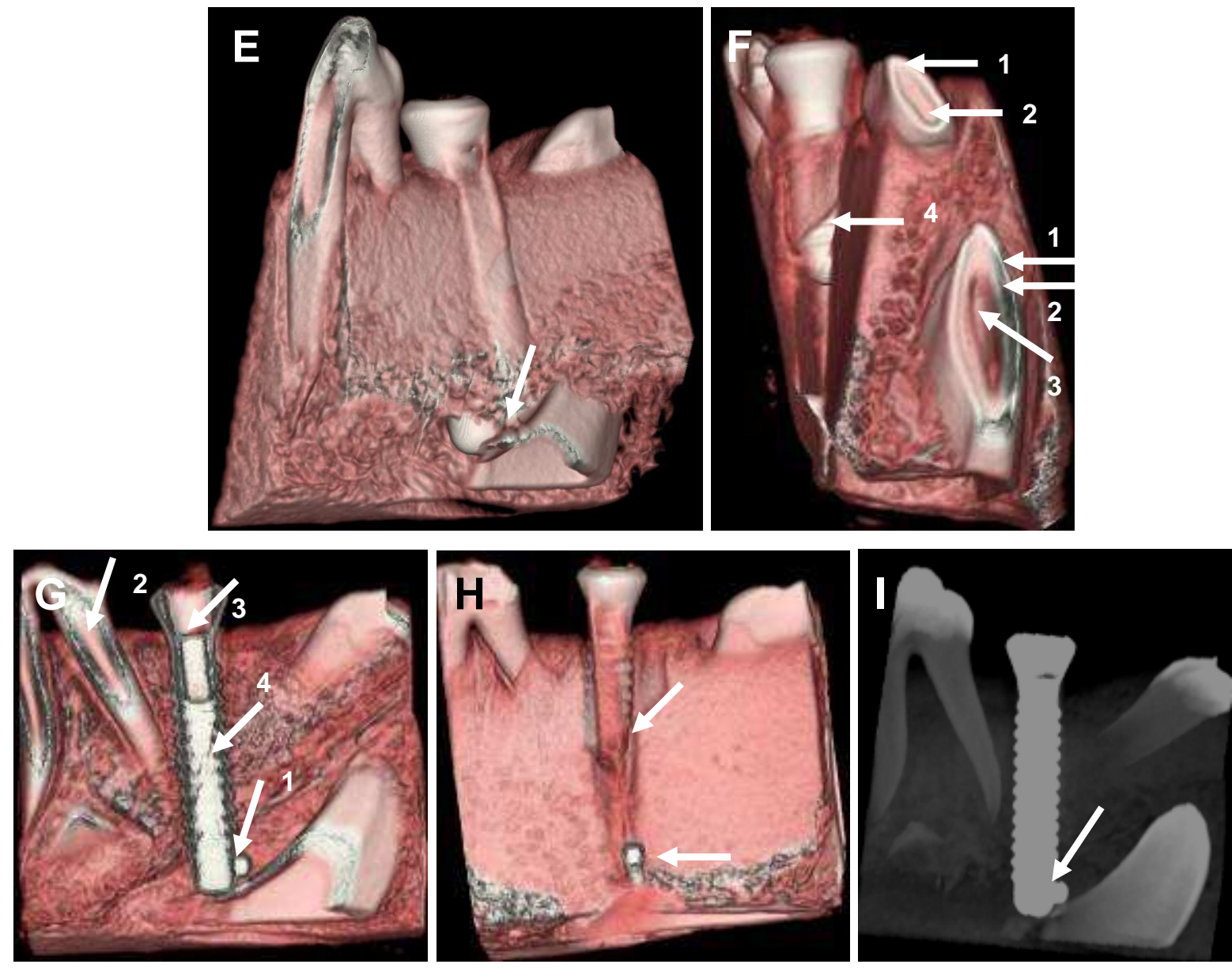

Abb. 15.2: Exemplarische Darstellung typischer Befunde der fpVCT (VR-Technik Volumendatensatz): E: Ansicht von vestibulär (Pfeil: Beziehung Implantat zu Zahnanlage), F: Ansicht von distal (Pfeil 1: Dentin, Pfeil 2: Zahnschmelz, Pfeil 3: Pulpa, Pfeil 4: linguale Perforation des Implantates, Implantat Nr. 14), G: Ansicht von vestibulär (Pfeil 1: räumliche Zuordnung einer Metallkugel zum Zahnimplantat, Pfeil 2: Pulpa, Pfeile 3+4: Innenleben des Implantates), $\boldsymbol{H + l : ~ A n s i c h t ~ v o n ~ v e s t i b u l a ̈ r ~}$ (räumliche Zuordnung einer Metallkugel zum Zahnimplantat (Pfeil). 


\subsection{Vergleich der radiologischen Verfahren}

\subsubsection{Vermessungen der Implantate}

Mit allen vier Verfahren waren Messungen zur Bestimmung von Länge und Durchmesser der Implantate möglich. Mit Hilfe einer Messlehre konnten im konventionellen Zahnfilm und im OPAN Werte ermittelt werden. Mit der entsprechenden Software der DVT (Professional Version der DVT 9000 Software) war eine Evaluation von Implantatlänge und-durchmesser ebenso möglich (Abb. 16.1). Bei der Befundung der fpVCT-Daten konnten im Transparentmodus II in Verbindung mit dem „Front-Cut-Modus“ die Implantate dargestellt und vermessen werden (Abb. 16.2).
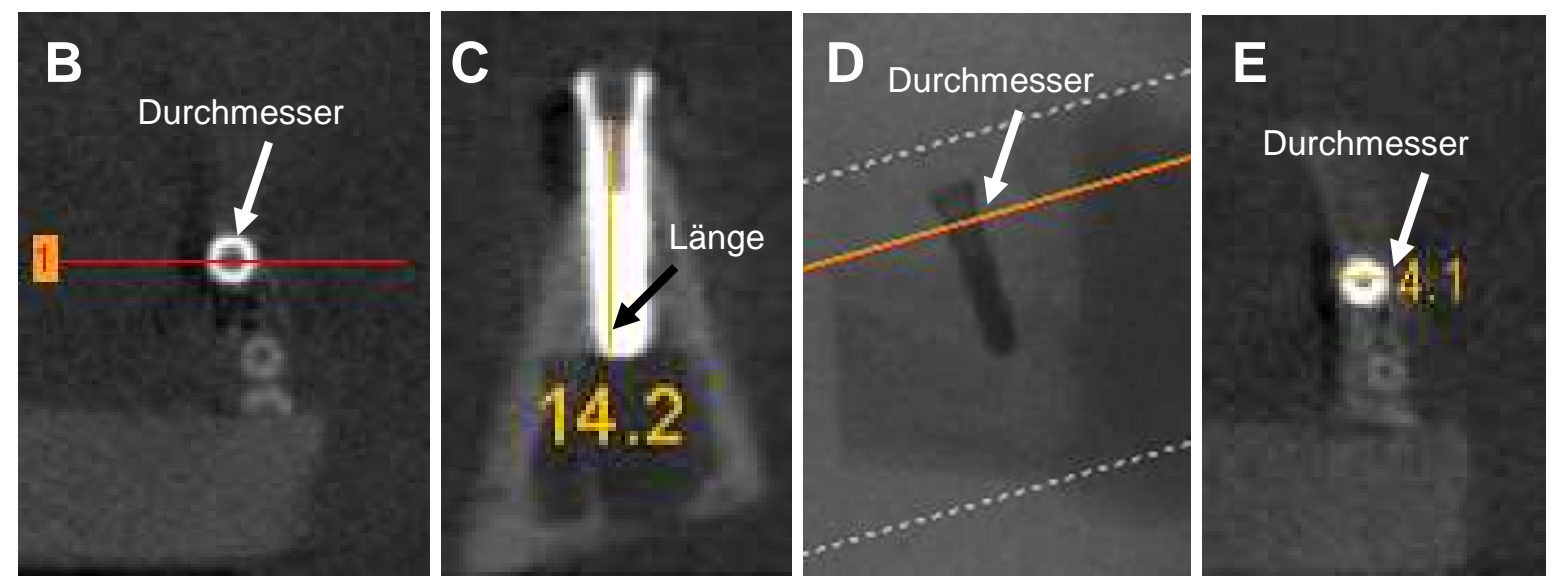

Abb. 16.1: DVT, Messungen von Implantatlänge und-durchmesser; siehe Pfeile (Präparat Nr. 8): B: transversale Schicht, rote Linie markiert die Auswahl der Schicht in der gemessen werden soll, C: koronare Schicht, gelbe Linie markiert den Anfangsund Endpunkt der Längenmessung mit Angabe des ermittelten Wertes in mm, D: orthoradiale Schicht, gelbe Linie markiert die Auswahl der Schicht in welcher der Durchmesser gemessen werden soll, E: transversale Schicht, gelbe Linie markiert den Anfangs- und Endpunkt der Messung des Durchmessers mit Angabe des ermittelten Wertes in $\mathrm{mm}$. 


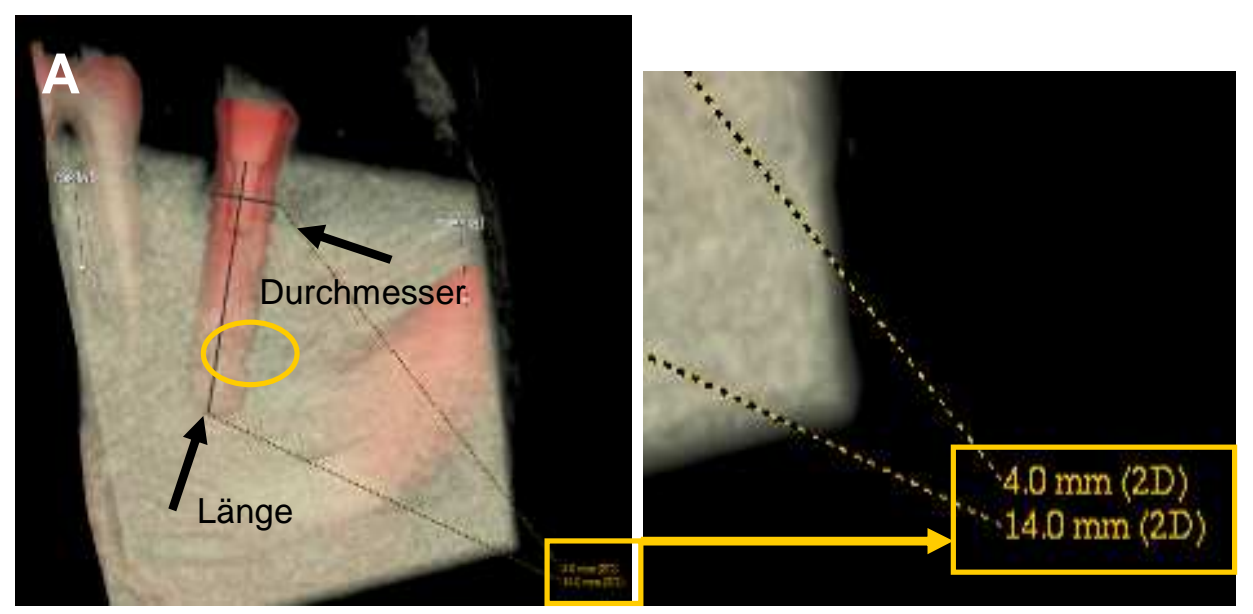

Abb. 16.2: fpVCT, Messungen von Implantatlänge und-durchmesser; siehe Pfeile (Präparat Nr. 8): A: VR-Technik Transparentmodus II, die blauen Linien markieren den Anfangs- und Endpunkt der beiden Messungen mit Angabe der ermittelten Werte in mm, Kreis: Querrille in Windungstiefe.

\subsubsection{Evaluation der Implantatlängen}

Mit allen Verfahren mit Ausnahme der Orthopantomographie konnten in mehr als zwei Dritteln die Implantatlängen durch die drei Untersucher den Herstellerangaben entsprechend bestimmt werden. Dabei wurden nur die Angaben als korrekt bewertet, die mit einer Abweichung von $\pm 0,1 \mathrm{~mm}$ mit denen des Herstellers übereinstimmten. Die Präzision der verschiedenen Verfahren unterschied sich signifikant voneinander $(p<0,0001 ;$ F-Test) (Abb. 17). 


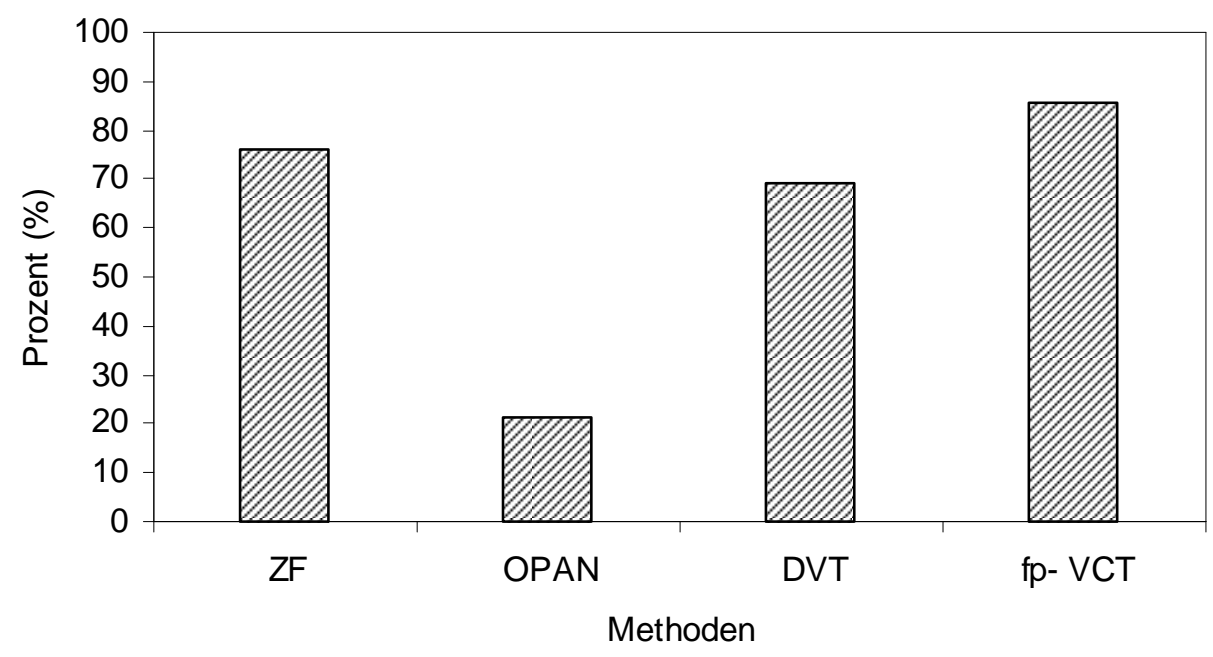

Abb. 17: Darstellung der Häufigkeit korrekt ermittelter Implantatlängen ( $Z F=Z a h n f i l m$, OPAN=Orthopantomographie, DVT=Digitale Volumentomographie, fpVCT=flatpanelVolumencomputertomographie).

Im Vergleich der vier Untersuchungsmethoden zeigten sich bei den Längenmessungen Abweichungen von den Originalgrößen von -1,3 mm bis $+4,7$ $\mathrm{mm}$. Dabei konnte mit der fpVCT die höchste Anzahl korrekter Befunde bei gleichzeitig geringstem Standardfehler erhoben werden. Des Weiteren wurden mit der OPAN die Implantate im Mittel um fast $2 \mathrm{~mm}$ zu lang vermessen. Im Einzelnen ergaben sich für die Messungen der Implantatlänge bei Implantat 1-14 für die einzelnen Aufnahmetechniken folgende Werte (Tab. 11):

\begin{tabular}{|l|cl|}
\hline Untersuchungsmethode & \multicolumn{2}{|c|}{$\begin{array}{c}\text { Messabweichungen bei Längenbestimmung } \\
\text { der Implantate 1 - 14 (Mittelwerte) }\end{array}$} \\
\hline Zahnfilm & $-0,18 \mathrm{~mm}$ & (Standardfehler $\pm 0,22)$ \\
\hline OPAN & $+1,81 \mathrm{~mm}$ & (Standardfehler $\pm 0,3)$ \\
\hline DVT & $+0,51 \mathrm{~mm}$ & (Standardfehler $\pm 0,21)$ \\
\hline fpVCT & $+0,05 \mathrm{~mm}$ & (Standardfehler $\pm 0,19)$ \\
\hline
\end{tabular}

Tab. 11: Tabellarische Darstellung der mittleren Messabweichungen bei der Längenbestimmung (Implantat 1 - 14) getrennt nach den verschiedenen Untersuchungsmethoden.

Im Folgenden ist die Verteilung der Differenzwerte der Implantatlängen anhand von Boxplots wiedergegeben (Abb. 18). Im Vergleich der vier Verfahren zeigten sich 
Unterschiede bezüglich der Verteilung der Messabweichungen. Bei der Orthopantomographie lag der Median der ermittelten Werte bei einer Messabweichung von $+2 \mathrm{~mm}$ und zeigt damit die im Mittel zu hohe Längenbestimmung in diesem Verfahren. Die maximale Messabweichung bezüglich zu hoher Längenangaben lag einmalig bei $6 \mathrm{~mm}$. Es wurde kein Implantat korrekt oder zu kurz gemessen. Bei den anderen drei Verfahren konnten die Werte im Mittel genau evaluiert werden mit einer maximalen Abweichung von weniger als $2 \mathrm{~mm}$. Lediglich bei der fpVCT kam es bei einer Implantatmessung zu einer größeren Messabweichung, wobei das Implantat $4 \mathrm{~mm}$ zu kurz gemessen wurde.

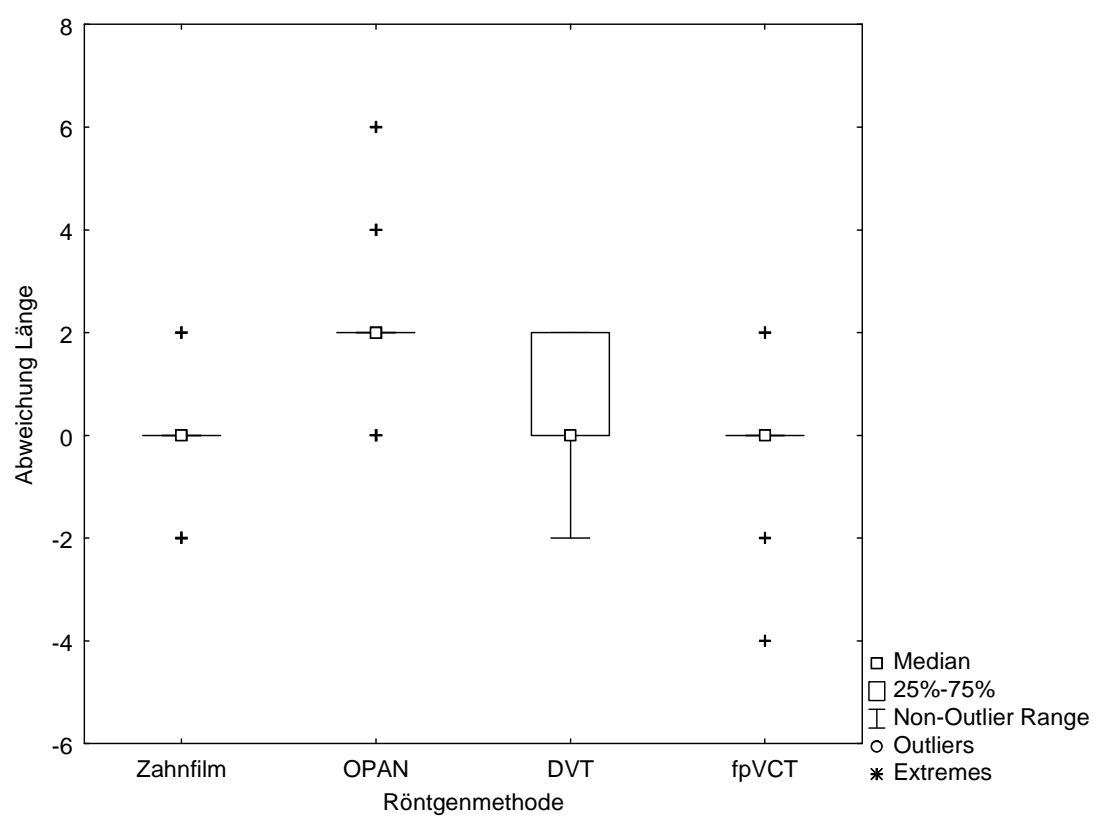

Abb. 18: Graphische Darstellung der Differenzwerte zu den Herstellerangaben der Längen aller 14 Implantate, getrennt nach den einzelnen Untersuchungsmethoden.

\subsubsection{Evaluation der Implantatdurchmesser}

Außerdem wurde die Bestimmung des Durchmessers mit den verschiedenen Methoden verglichen (Abb. 19). Es lag kein signifikanter Unterschied zwischen den Verfahren vor $(p=0,1523$; F-Test). 


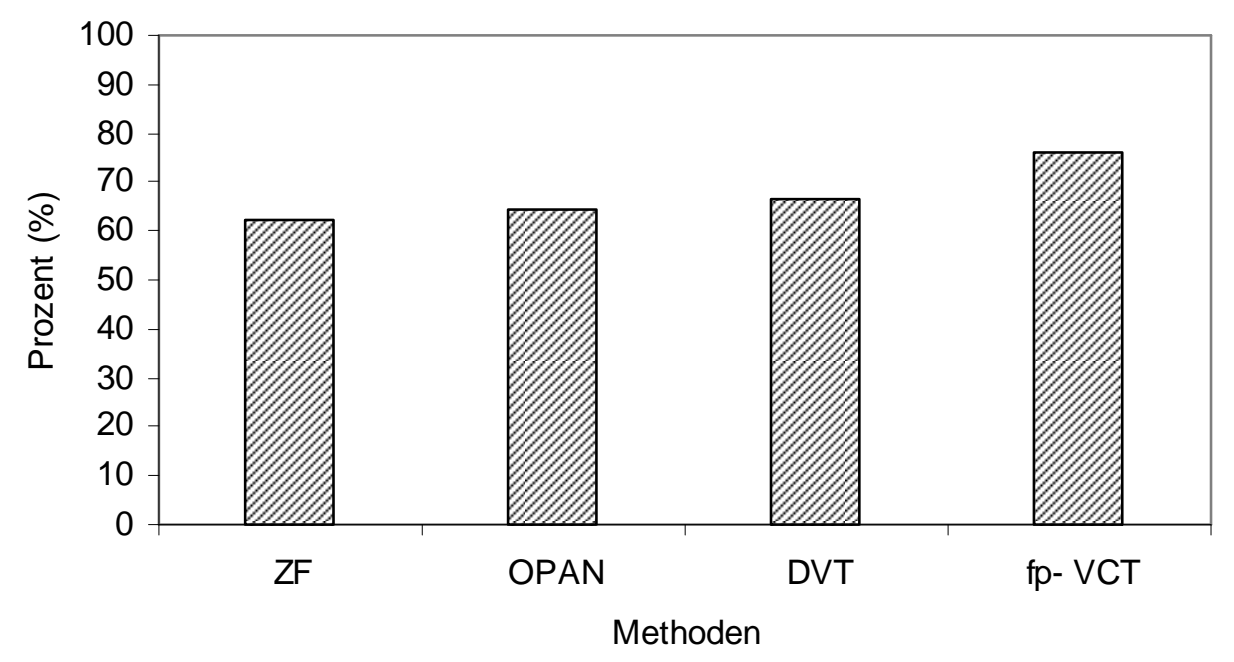

Abb. 19: Darstellung der Häufigkeit korrekt festgestellter Implantatdurchmesser (ZF=Zahnfilm, OPAN=Orthopantomographie, DVT=Digitale Volumentomographie, fpVCT=flatpanel-Volumencomputertomographie).

Bei der Evaluation der vier Verfahren in Bezug auf die Durchmesser zeigten sich Abweichungen von $-0,53$ bis $+0,27 \mathrm{~mm}$. Im Einzelnen ergaben sich für die Messungen der Implantatdurchmesser bei Implantat 1-14 für die einzelnen Aufnahmen folgende Werte (Tab. 12):

\begin{tabular}{|l|ll|}
\hline Untersuchungsmethode & \multicolumn{2}{|c|}{$\begin{array}{c}\text { Messabweichungen der Durchmesserbestimmung } \\
\text { der Implantate 1 - 14 (Mittelwerte) }\end{array}$} \\
\hline Zahnfilm & $-0,125 \mathrm{~mm}$ & (Standardfehler $\pm 0,05)$ \\
\hline OPAN & $+0,0079 \mathrm{~mm}$ & (Standardfehler $\pm 0,04)$ \\
\hline DVT & $+0,02 \mathrm{~mm}$ & (Standardfehler $\pm 0,03)$ \\
\hline fpVCT & $+0,0093 \mathrm{~mm}$ & (Standardfehler $\pm 0,01)$ \\
\hline
\end{tabular}

Tab. 12: Tabellarische Darstellung der mittleren Messabweichungen bei der Durchmesserbestimmung (Implantat 1 - 14) getrennt nach den verschiedenen Untersuchungsmethoden.

Im Folgenden ist die Verteilung der Messungen der Implantatdurchmesser anhand von Boxplots dargestellt (Abb. 20). Bei allen Verfahren liegt der Median bei dem korrekt ermittelten Messwert. Sowohl in Zahnfilm und OPAN als auch in der DVT ist es vereinzelt zu Messabweichungen von bis zu $0,8 \mathrm{~mm}$ gekommen. Bei der DVT ist 
ein Implantat um $1 \mathrm{~mm}$ zu dünn vermessen worden. Hingegen lagen in der fpVCT die Ausreißer bei einer Differenz vom Zielwert von maximal 0,1 mm.

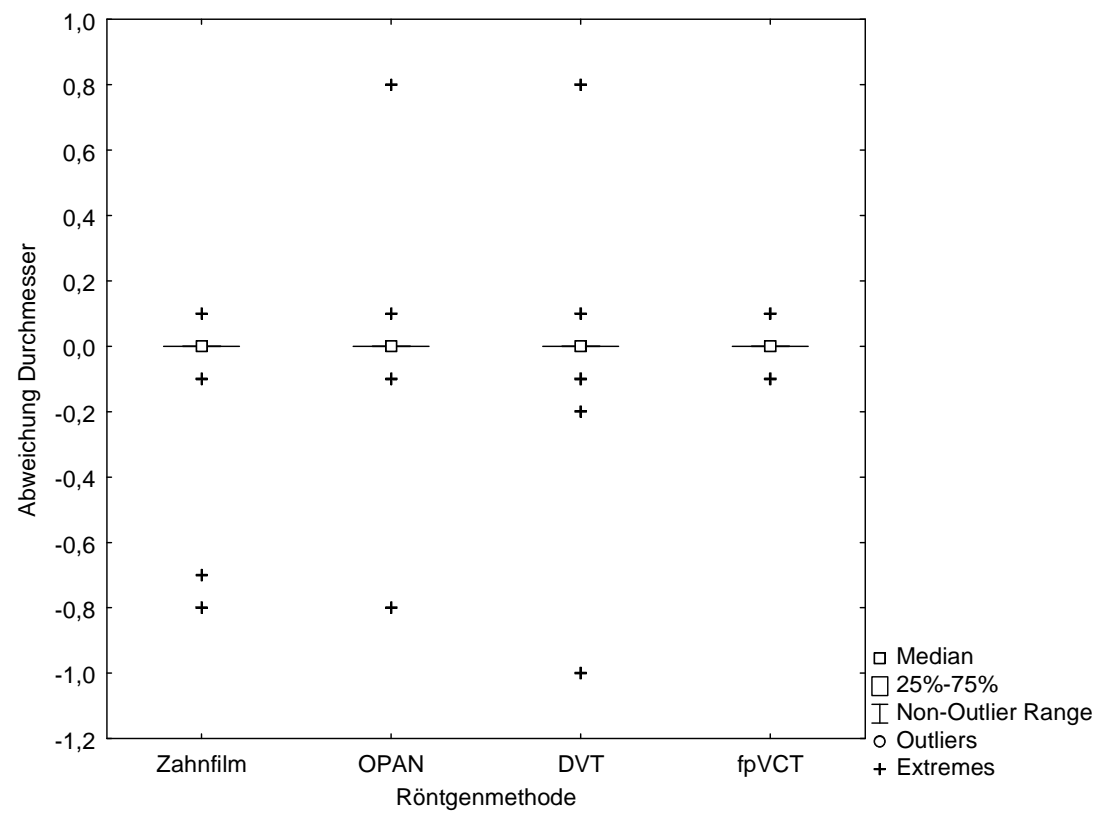

Abb. 20: Graphische Darstellung der Differenzwerte zu den Herstellerangaben der Durchmesser aller 14 Implantate, getrennt nach den einzelnen

Untersuchungsmethoden.

\subsubsection{Evaluation der iatrogen applizierten Knochen- bzw. Implantatdefekte und Fremdkörper}

Außerdem wurde untersucht, wie viele Untersucher bei Anwendung der einzelnen Röntgenmethoden das Vorhandensein oder Fehlen eines iatrogen applizierten Alveolar- resp. Implantatdefektes oder eines Fremdkörpers korrekt erkannt haben.

\subsubsection{Knochendefekte}

Bei der Evaluation der Knochendefekte unterschieden sich die vier Verfahren statistisch nicht signifikant voneinander ( $p=0,5034$; F-Test). Während mit den zweidimensionalen Verfahren in 62\% der Fälle der Knochendefekt genau diagnostiziert werden konnte, wurde mit der fpVCT in 57\% ein Defekt korrekt erkannt. Mit der DVT konnten 50\% der Defekte korrekt befundet werden. 
Der Defekt X (1 mm tiefe keilförmige Bohrung in $4 \mathrm{~mm}$ Höhe) war fast ausschließlich durch die dreidimensionalen Verfahren detektierbar, wohin gegen der Defekt $Y$ (Längsrille von $1 \mathrm{~mm}$ Tiefe und $\leq 10 \mathrm{~mm}$ Länge) auch mit Hilfe der zweidimensionalen Methoden erfasst wurde (Abb. 21.1 und 21.2). Mit DVT und fpVCT gelang es den Untersuchern, die Art des Defektes genau zuzuordnen, während mit Zahnfilm und OPAN lediglich Beschreibungen periimplantärer knöcherner Strukturveränderungen und Veränderungen der Röntgendichte möglich waren. So wurde der Knochendefekt Y (Schweinekieferprobe Nr.7) anhand der Zahnfilmaufnahme als eine $2 \mathrm{~mm}$ tiefe und $1 \mathrm{~mm}$ breite Aufhellung distal im Bereich der Implantattulpe beschrieben während an der OPAN-Aufnahme eine $1 \mathrm{~mm}$ tiefe Läsion mesial etwa $9 \mathrm{~mm}$ unterhalb der Implantattulpe beschrieben wurde. Der Knochendefekt X (Schweinekieferprobe Nr. 9) wurde anhand der Zahnfilmaufnahme als 2,5 mm langer Spalt distal zwischen Implantat-Knochenverband gesehen, die OPAN-Aufnahme ließ keine Beurteilung des Defektes zu.
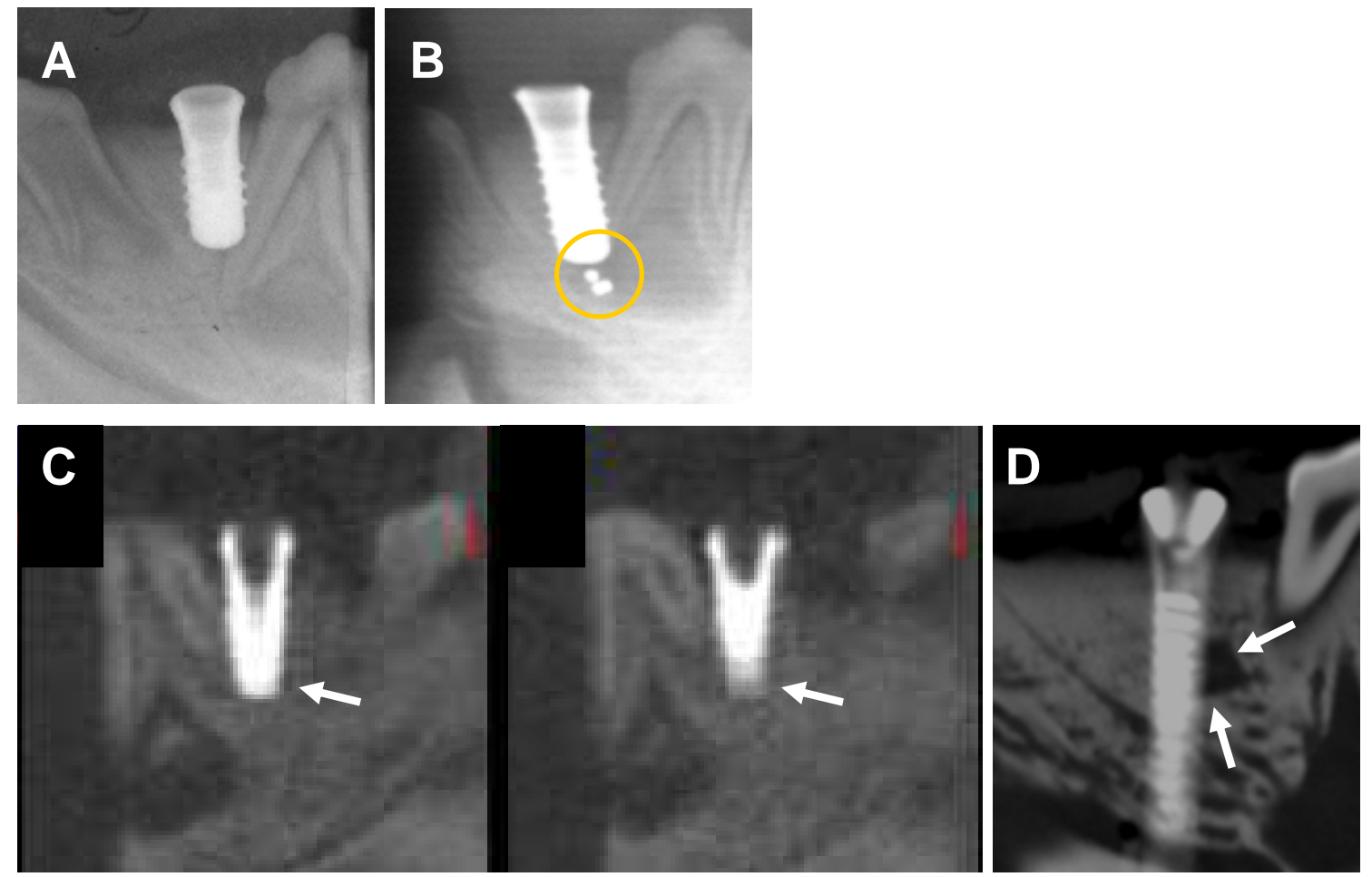

Abb. 21.1: Knochendefekt $X$ (siehe Pfeile): $1 \mathrm{~mm}$ tiefe keilförmige Bohrung in $4 \mathrm{~mm}$ Höhe; A: Zahnfilm (Präparat Nr. 6), B: Ausschnitt aus OPAN (Präparat Nr. 9) Kreis: zusätzlich 3 Metallkugeln von je $1 \mathrm{~mm}$ Durchmesser, C: DVT (Präparat Nr. 6), D: fpVCT (Präparat Nr. 6). 

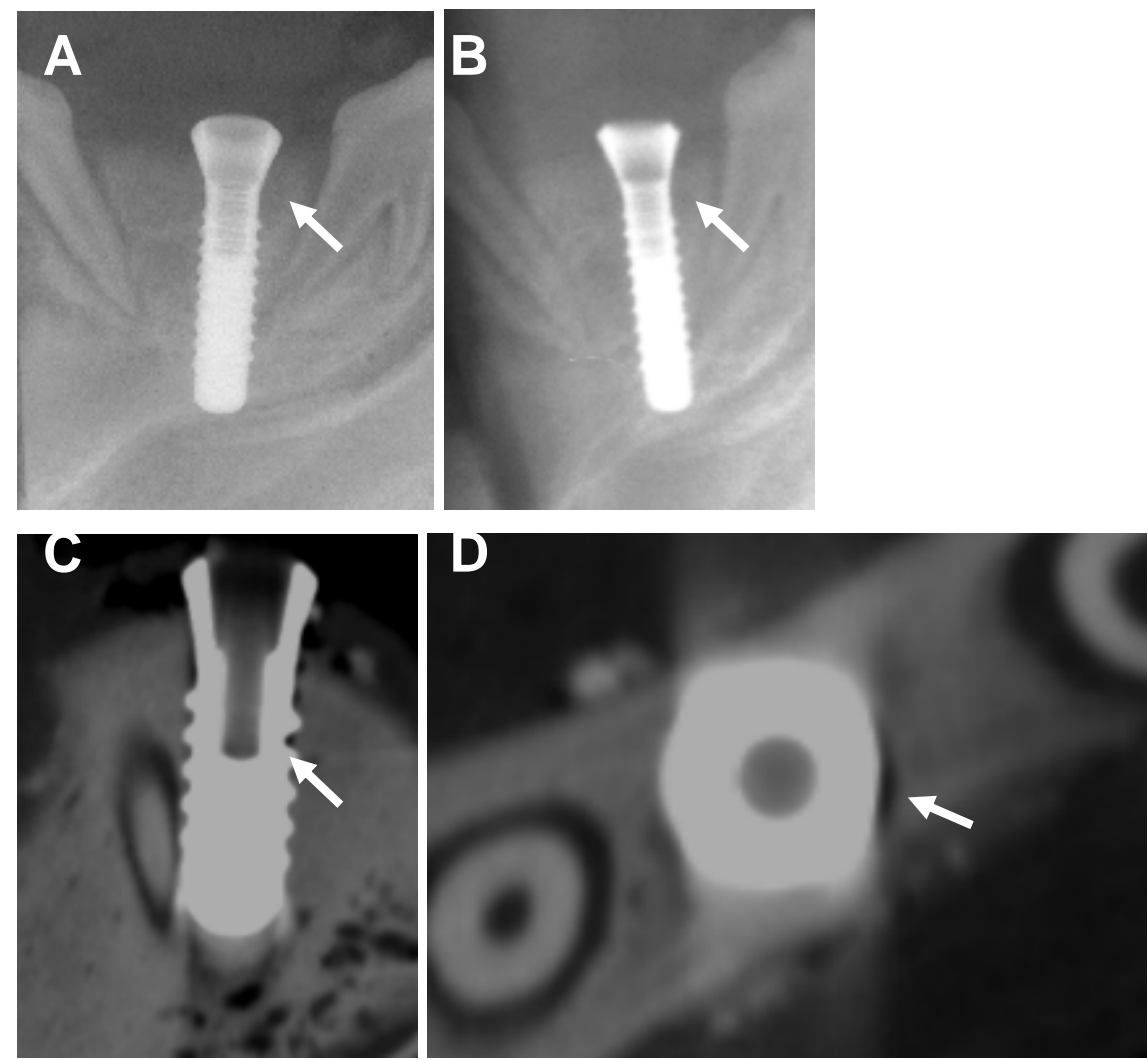

Abb. 21.2: Knochendefekt $Y$ (siehe Pfeile): Längsrille von $1 \mathrm{~mm}$ Tiefe und $\leq 10 \mathrm{~mm}$ Länge; A: Zahnfilm (Präparat Nr. 7), B: Ausschnitt aus OPAN (Präparat Nr. 7), C+D: fpVCT (Präparat Nr. 12).

Das bei der Implantatinsertion des Präparates 10 durchgeführte Bonesplitting wurde in den zweidimensionalen Verfahren als eine Aufhellung beschrieben und die Untersucher werteten es als Veränderung der Knochenstruktur im Bereich des Implantationsgebietes (Abb. 22.1). Eine Größenbestimmung der Strukturveränderung konnte lediglich anhand der OPAN-Aufnahme getroffen werden. Die DVT ließ keine Aussagen über diesen Defekt zu. Die fpVCT zusammen mit der Volume-RenderingTechnik ermöglichte eine exakte Beurteilung der knöchernen Fragmente hinsichtlich Größe und Lokalisation insbesondere auch in Relation zum Implantat (Abb. 22.2). 

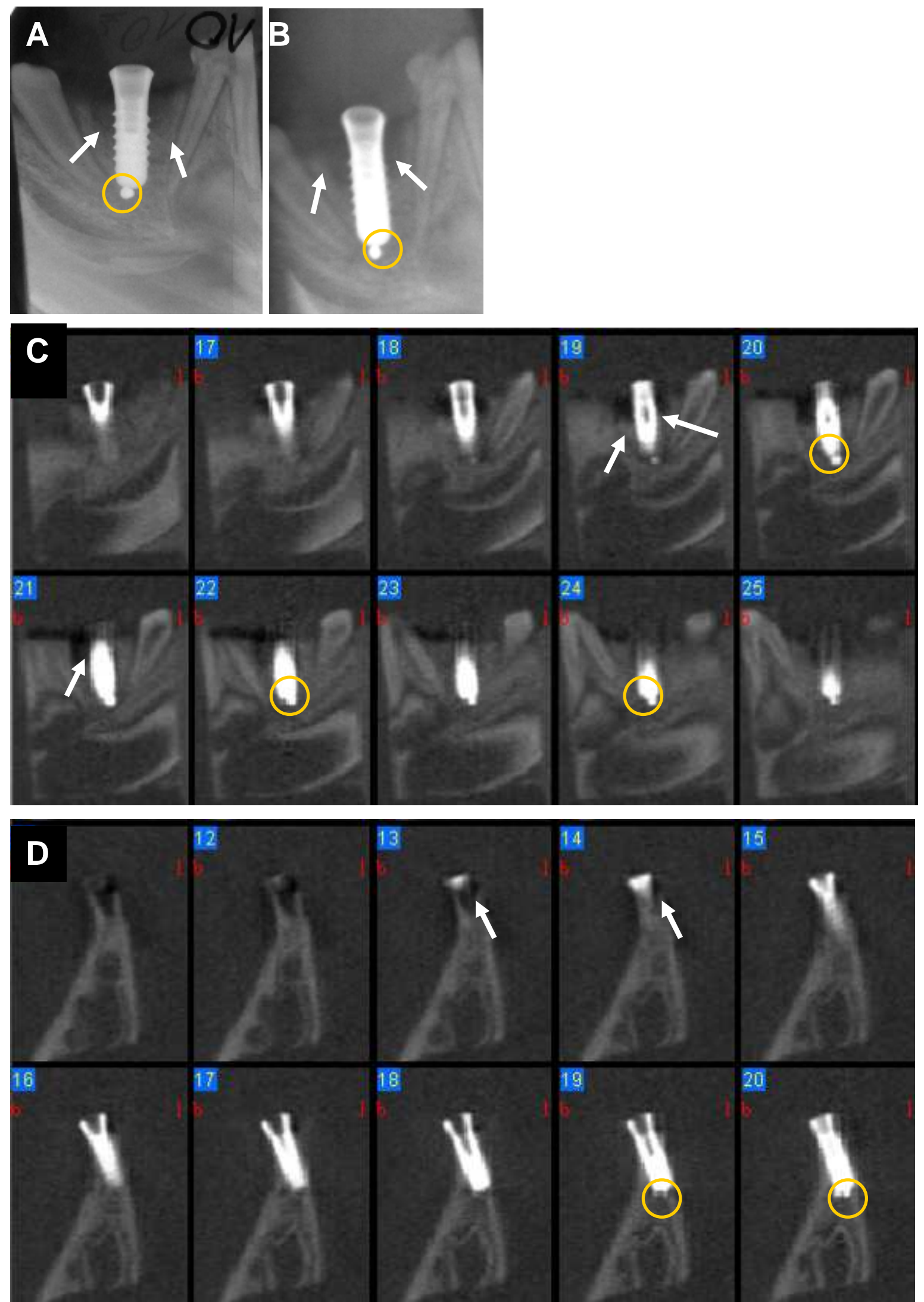

Abb. 22.1: Knochendefekt: Bonesplitting Implantat Nr. 10; Pfeile: markieren die Frakturlinien der Knochenfragmente, Kreise: 1 Metallkugel je $1 \mathrm{~mm}$ und 1,6 mm Durchmesser; A: Zahnfilm, B: Ausschnitt aus OPAN, C: DVT orthoradiale Schicht, D: DVT koronale Schicht. 

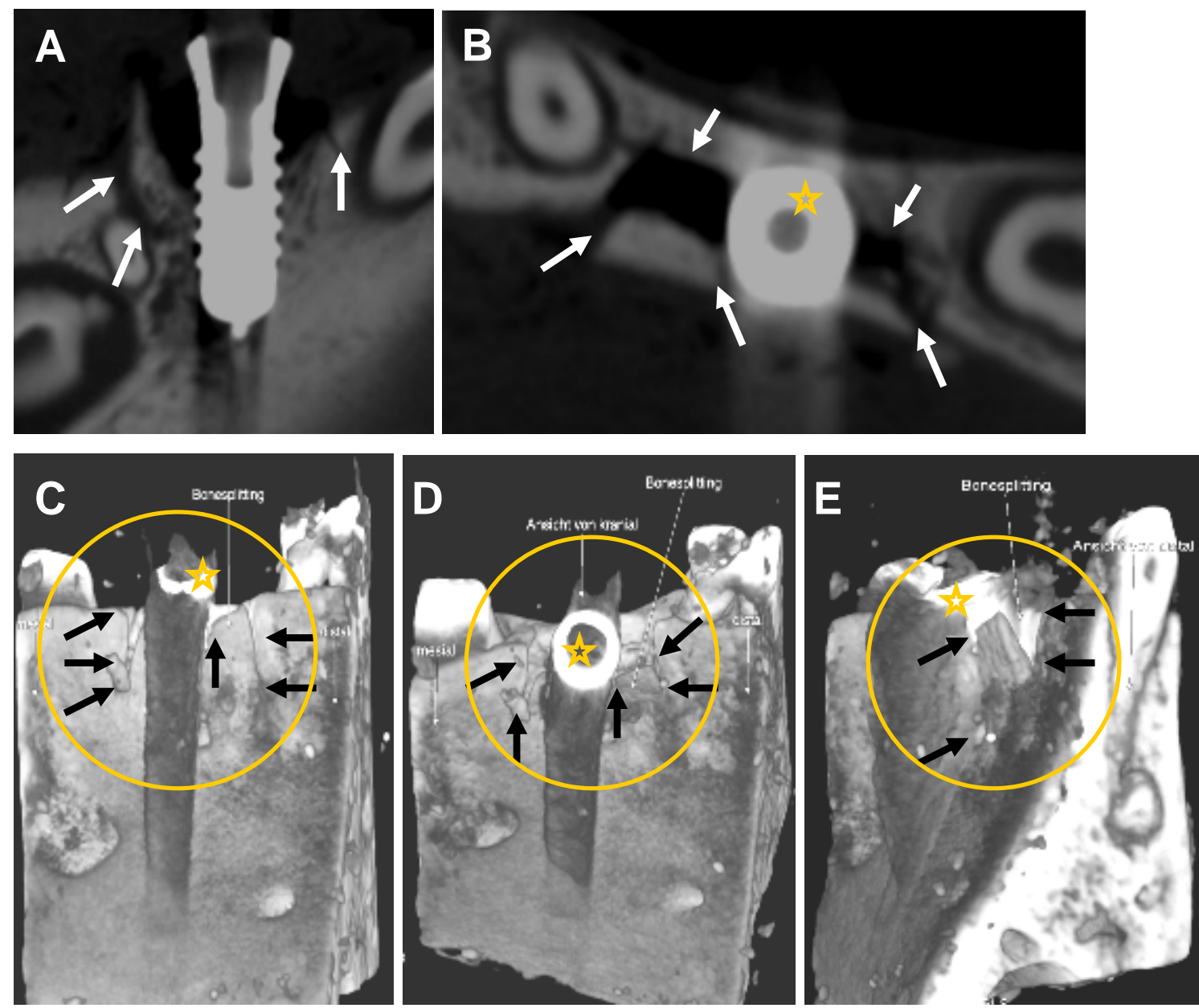

Abb. 22.2: Knochendefekt: Bonesplitting (siehe Kreis) Präparat Nr. 10; fpVCT VRTechnik; Pfeile markieren die Frakturlinien der einzelnen Knochenfragmente, A: orthoradiale Schicht, B: transversale Schicht, Stern: Dentalimplantat im Bereich Implantattulpe, C-D: 3D-Rekonstruktion Volumendatensatz, Stern: Dentalimplantat, C: Ansicht von lingual, D: Ansicht von kranial, E: Ansicht von distal lateral.

Bei Präparat 14 kam es während der Implantatinsertion lingual zu einer Perforation des Alveolarkammes. Mit Zahnfilm, OPAN und DVT konnte der Defekt als ein Verlust des Implantat-Knochen-Kontaktes beschrieben werden. Eine räumliche Zuordnung gelang dabei in zwei Ebenen. Mit Hilfe der fpVCT hingegen war eine exakte Beurteilung des Defektes möglich. Mit der Volume-Rendering-Technik gelang zudem die räumliche Zuordnung des Defektes insbesondere in Relation zum Implantat (Abb. 23.1 und 23.2). 


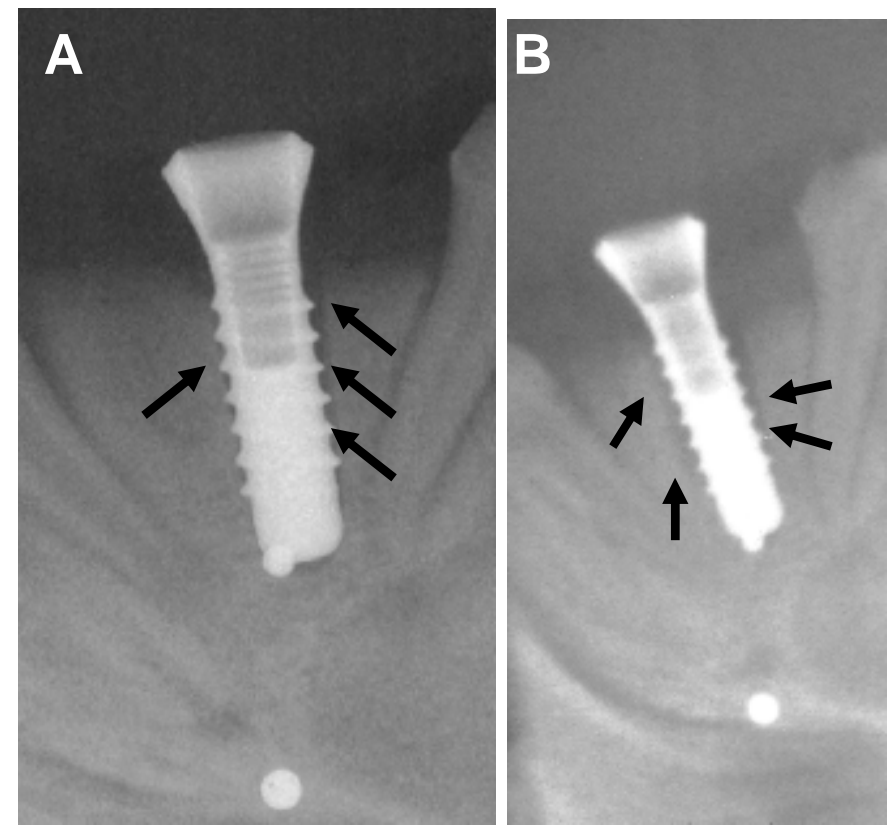

Abb. 23.1: Knochendefekt: Knochenperforation, Präparat Nr.14, Pfeile: Markierung der Frakturlinien; $\boldsymbol{A}:$ Zahnfilm, B: OPAN.
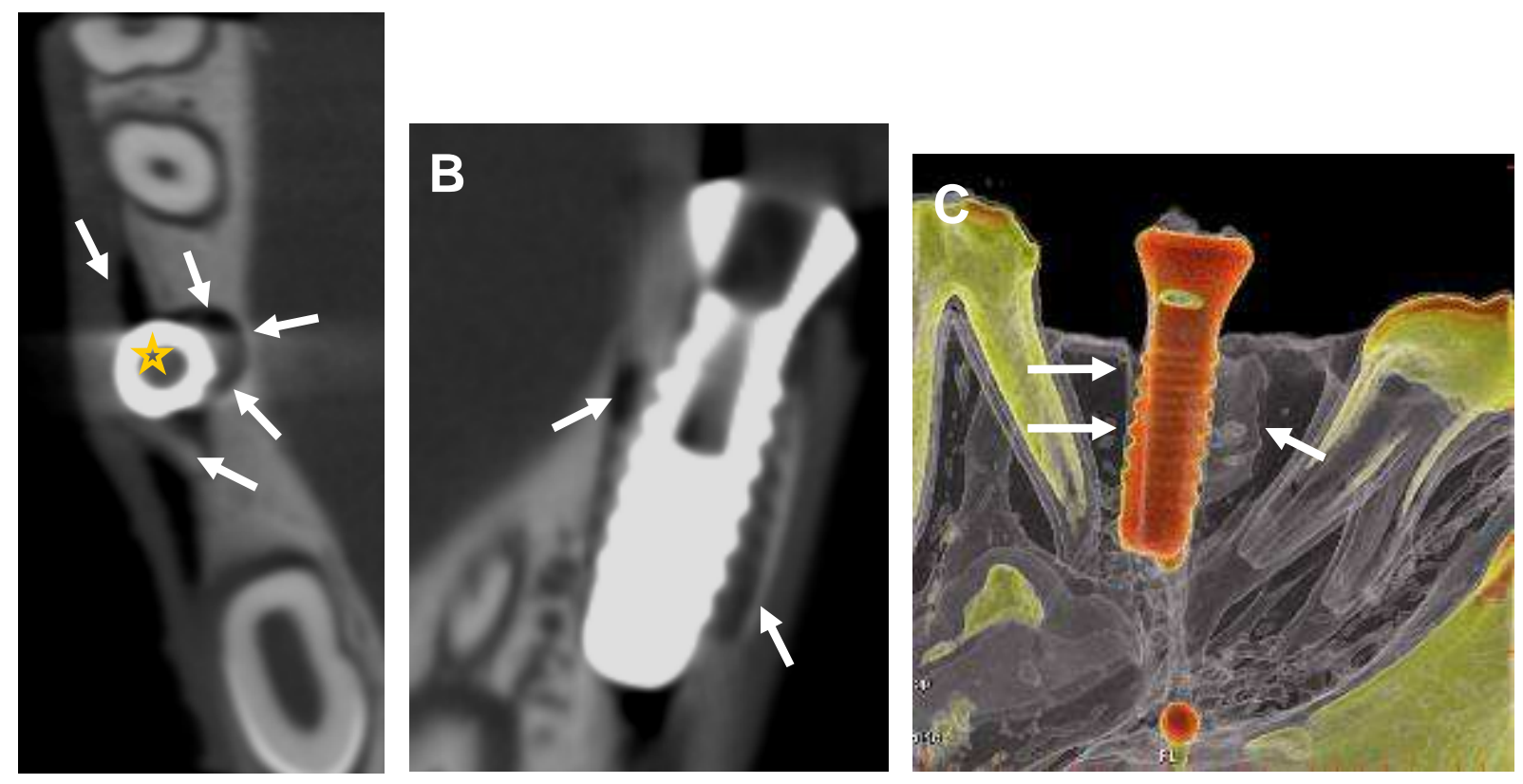

Abb. 23.2: Knochendefekt: Knochenperforation, fpVCT VR-Technik (Präparat Nr. 14), Pfeile: Markierung der Frakturlinien und der knöchernen Begrenzung der vorpräparierten Kavität, A: transversale Schicht, Stern: Implantatkopf, C: Transparentmodus $I$.

Als ein zusätzlicher Defekt wurde bei Präparat Nr. 13 die Kavität für das Implantat im Rahmen der Vorbohrungen im Verhältnis zur Implantatgröße nach apikal 
überdimensioniert (Abb. 24). Diese zu tiefe Kavität war mit allen Verfahren detektierbar. Die Breite und die Tiefenausdehnung konnten in allen Fällen beschrieben werden.
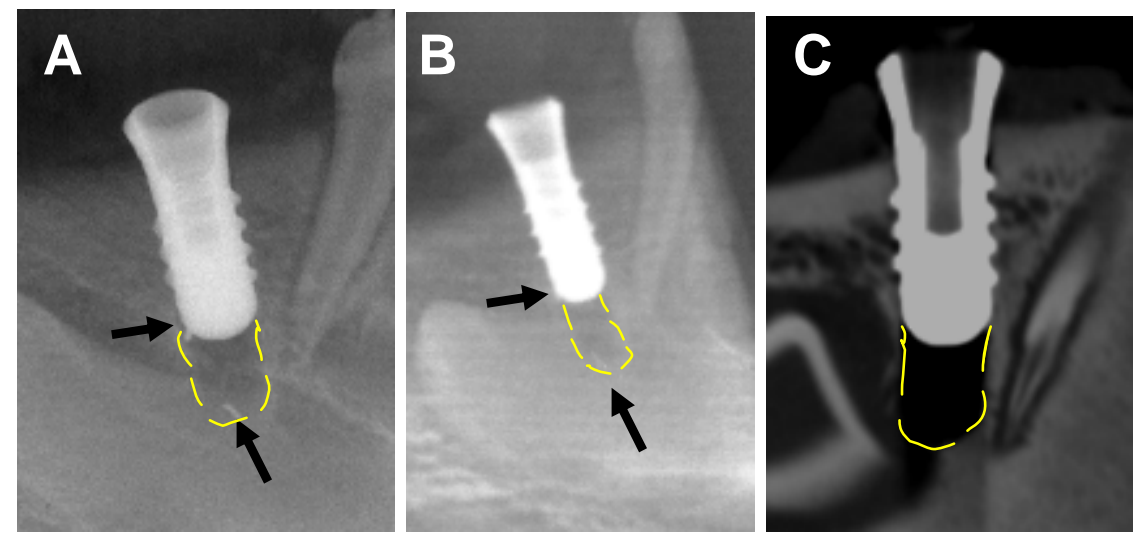

Abb. 24: Knochendefekt: Überdimensionierte Kavität (gelbe Linie) Präparat Nr. 13, Pfeile: Guttaperchastifte; A: Zahnfilm, B: OPAN, C: fpVCT.

\subsubsection{Implantatdefekte}

Mit allen vier Verfahren waren Implantatdefekte darstellbar. Bei der Detektion der Implantatdefekte zeigten sich ebenfalls keine statistisch signifikanten Unterschiede im intermethodischen Vergleich ( $p=0,3212 ; \mathrm{F}$-Test). In annähernd $70 \%$ der Fälle konnten mittels Zahnfilm und fpVCT die Defekte korrekt befundet werden. Bei OPAN und DVT ergaben sich in annähernd $60 \%$ korrekte Befunde.

\section{Längsrille:}

Mit der Zahnfilmaufnahme und der fpVCT-Methode konnte die Längsrille im Windungsschaft des Implantates durch die Untersucher evaluiert werden (Abb. 25).

Die Lokalisation und Größendimension der Längsrille wurden korrekt befundet. OPAN und DVT ließen eine Darstellung dieses Implantatdefektes nicht zu. 


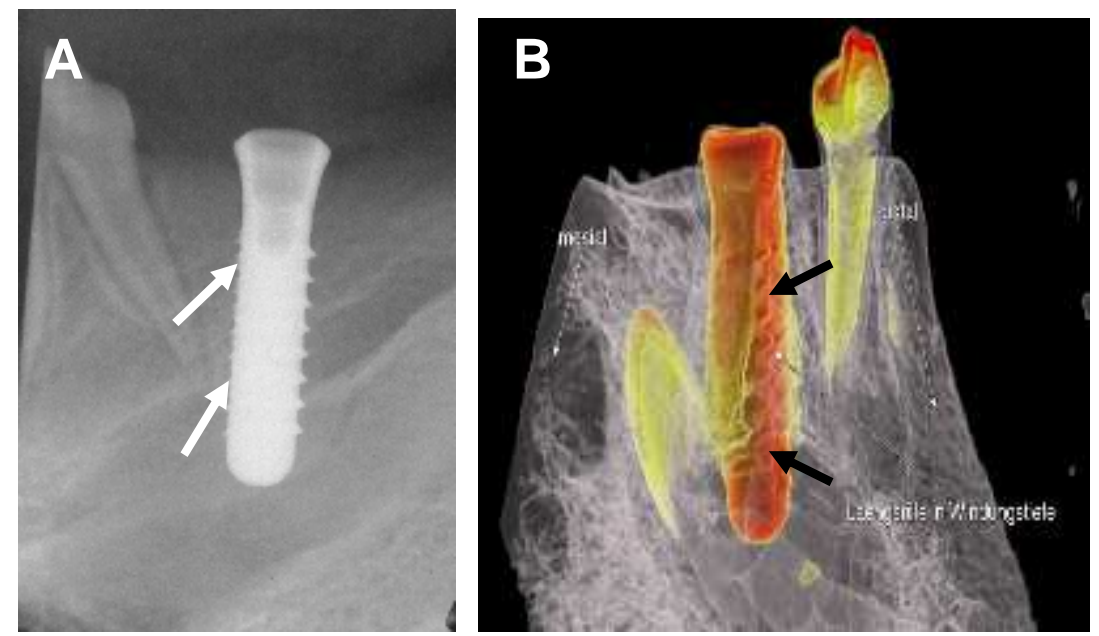

Abb. 25: Längsrille in Windungstiefe (siehe Pfeile) Präparat Nr. 5; A: Zahnfilm, B: fpVCT VR-Technik Transparentmodus I.

\section{Querrille:}

Bei zwei Implantaten lag ein Defekt in Form einer Querrille im Windungsschaft vor. Die gewonnen Bilddaten ließen dabei nur an einem der beiden Implantate eine Beurteilung des Defektes mit allen Verfahren zu. Im zweiten Fall war eine Detektion des Defektes nur mit der fpVCT möglich (Abb. 26.1 und 26.2).

Die Lokalisation und Größe des Defektes wurde in allen Fällen korrekt beurteilt.
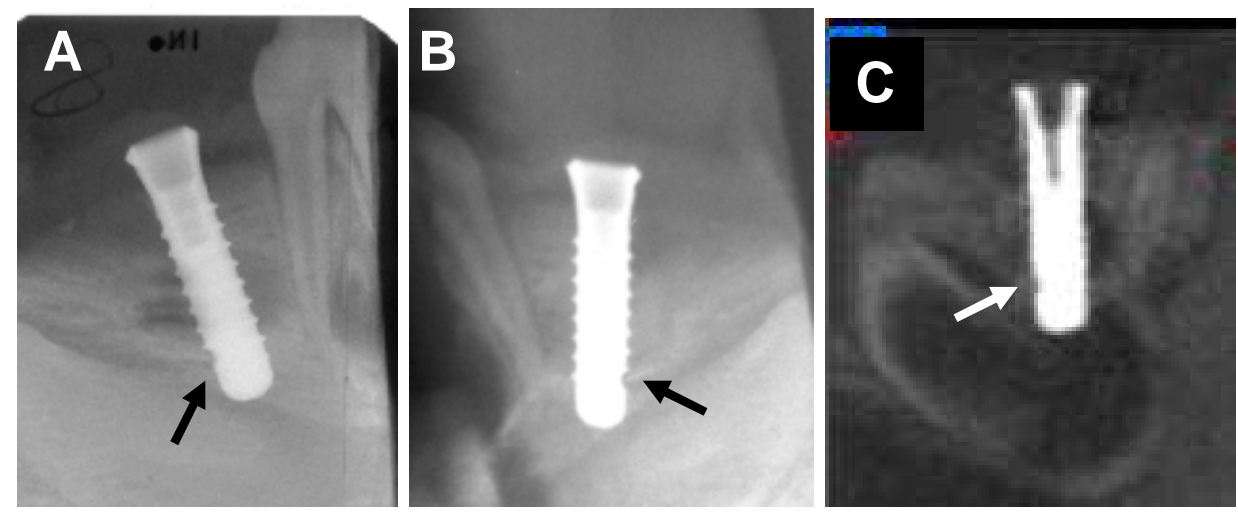

Abb. 26.1: Querrille in der Windungstiefe (siehe Pfeil) Präparat Nr. 8; A: Zahnfilm, B: OPAN, C: DVT. 

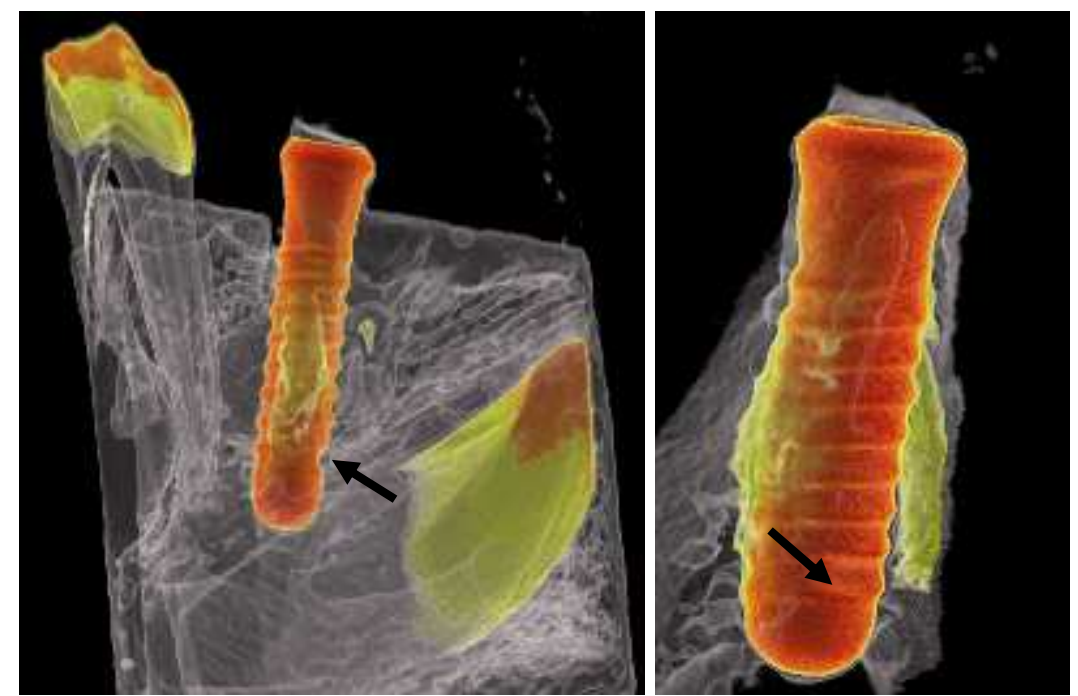

Abb. 26.2: Querrille in Windungstiefe (siehe Pfeil) Präparat Nr. 8; fpVCT VR-Technik Transparentmodus $I$.

Planierung der Implantatoberflächen:

Zwei Implantate wiesen einen Defekt in Form einer Planierung von 1-2

Gewindegängen auf. Bei dem Implantat Nr. 1 konnte der Defekt durch die zweidimensionalen Verfahren korrekt detektiert werden, bei dem Implantat Nr. 3 war eine Beurteilung der Planierung nur mittels fpVCT möglich (Abb. 27). Mit der DVT war keine Beurteilung der Gewindegänge möglich.
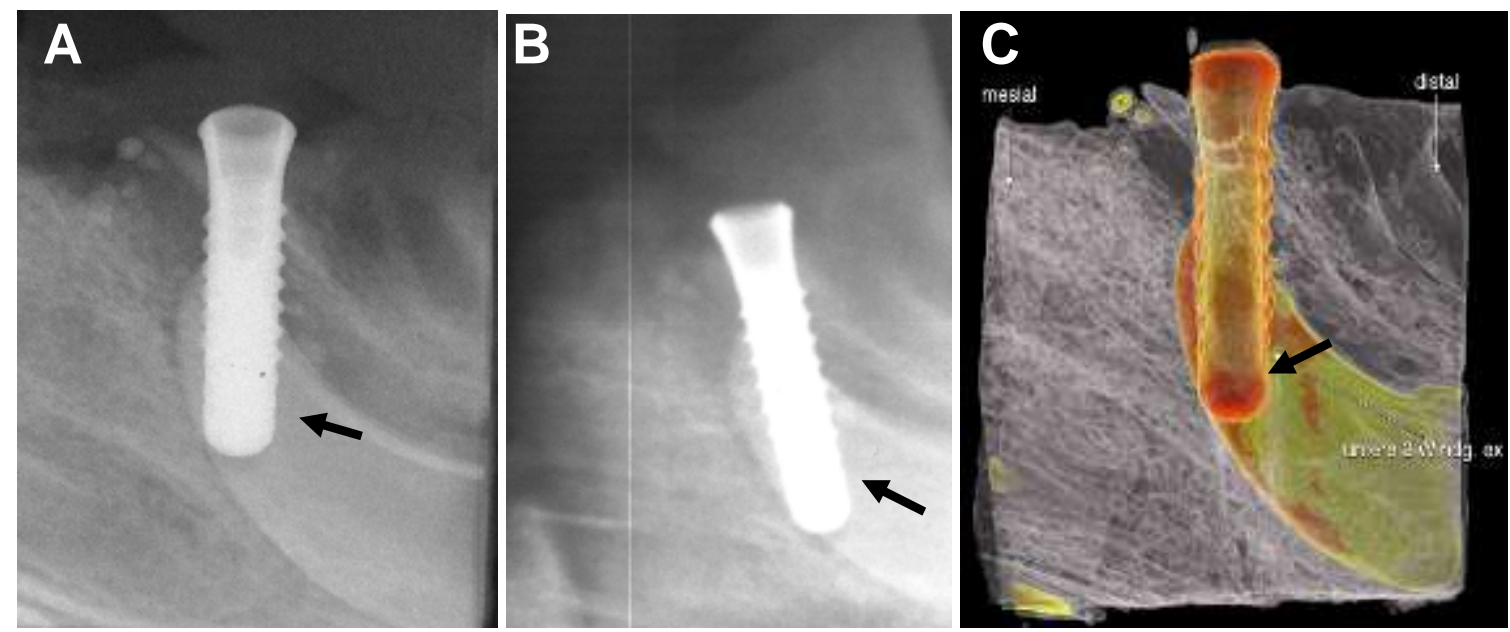

Abb. 27: Planierte Gewindegänge (siehe Pfeil) Präparat Nr. 1; A: Zahnfilm, B: OPAN, C: fpVCT VR-Technik Transparentmodus I. 


\subsubsection{Fremdkörper}

Es wurden Fremdkörper aus Werkstoffen unterschiedlicher Röntgenopazität (Stahl, Guttapercha, B-TCP) untersucht. Mit allen Methoden war eine Darstellung der verschiedenen Fremdmaterialien möglich. In über $80 \%$ aller Befunde konnten mit den vier Methoden genaue Angaben über das Vorhandensein eines oder mehrerer Fremdkörper getroffen werden. Die Unterschiede zwischen den einzelnen Röntgenmethoden waren statistisch nicht signifikant ( $p=0,2258$; F-Test).

Metallkugel:

Insgesamt wurden 10 kugelförmige Metallobjekte vor der Implantatinsertion in vorgebohrte Kavitäten der Präparate eingebracht. Dabei wurde in 2 Fällen (Präparat 2 und 4) jeweils eine einzelne Metallkugel der Größe $1 \mathrm{~mm}$ verwendet, in einem Fall (Präparat 11) eine einzelne Kugel der Größe 1,6 mm. Bei Präparat 10 und 14 wurden jeweils eine kleine und eine große Metallkugel inseriert. In die Kavität von Präparat 9 wurden 3 Metallkugeln mit $1 \mathrm{~mm}$ Größe eingefügt.

Die kugelförmigen Metallobjekte konnten stets mit allen bildgebenden Verfahren korrekt befundet werden (Tab. 13 und Abb. 28.1 - 28.4). 


\begin{tabular}{|c|c|c|c|c|c|c|c|}
\hline & \multicolumn{6}{|c|}{$\begin{array}{l}\text { Häufigkeit (Anzahl) der zutreffenden Größenbestimmungen der Metallkugeln (MK) } \\
\text { an } 6 \text { verschiedenen Präparaten durch } 3 \text { Untersucher }\end{array}$} \\
\hline & & $\begin{array}{l}\text { Präparat } 2 \\
1 \mathrm{MK}: \\
\varnothing 1 \mathrm{~mm}\end{array}$ & $\begin{array}{l}\text { Präparat } 4 \\
1 \mathrm{MK}: \\
\varnothing 1 \mathrm{~mm}\end{array}$ & $\begin{array}{l}\text { Präparat } 11 \\
1 \mathrm{MK}: \\
\varnothing 1,6 \mathrm{~mm}\end{array}$ & $\begin{array}{l}\text { Präparat } 10 \\
1 \mathrm{MK}: \\
\varnothing 1 \mathrm{~mm} \text { und } \\
1 \mathrm{MK}: \\
\varnothing 1,6 \mathrm{~mm}\end{array}$ & $\begin{array}{l}\text { Präparat } 14 \\
1 \mathrm{MK}: \\
\varnothing 1 \mathrm{~mm} \text { und } \\
1 \mathrm{MK}: \\
\varnothing 1,6 \mathrm{~mm}\end{array}$ & $\begin{array}{l}\text { Präparat } 9 \\
3 \mathrm{MK}: \\
\varnothing 1 \mathrm{~mm}\end{array}$ \\
\hline \multirow{4}{*}{ 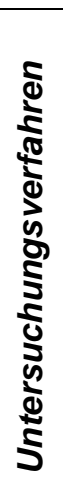 } & ZF & 2 & 3 & 3 & $\begin{array}{l}3(1 \mathrm{~mm}) \\
3(1,6 \mathrm{~mm})\end{array}$ & $\begin{array}{l}2(1 \mathrm{~mm}) \\
3(1,6 \mathrm{~mm})\end{array}$ & 6 \\
\hline & OPAN & 1 & 3 & 3 & $\begin{array}{l}1(1 \mathrm{~mm}) \\
2(1,6 \mathrm{~mm})\end{array}$ & $\begin{array}{l}2(1 \mathrm{~mm}) \\
3(1,6 \mathrm{~mm})\end{array}$ & 5 \\
\hline & DVT & 1 & 1 & 2 & $\begin{array}{l}3(1 \mathrm{~mm}) \\
2(1,6 \mathrm{~mm})\end{array}$ & $\begin{array}{l}2(1 \mathrm{~mm}) \\
3(1,6 \mathrm{~mm})\end{array}$ & 2 \\
\hline & fpVCT & 1 & 1 & 3 & $\begin{array}{l}3(1 \mathrm{~mm}) \\
3(1,6 \mathrm{~mm})\end{array}$ & $\begin{array}{l}3(1 \mathrm{~mm}) \\
3(1,6 \mathrm{~mm})\end{array}$ & 9 \\
\hline
\end{tabular}

Tab. 13: Häufigkeit der korrekten Befundung unterschiedlich großer Metallkugeln (1 mm und 1,6 mm) und unterschiedlicher Kombinationen der verschiedenen Größe und Anzahl von Metallkugeln mit den verschiedenen bildgebenden Verfahren durch 3 Untersucher.

Die Beurteilung von Anzahl und Größe der Metallkugeln war mit allen Verfahren gut durchführbar. Teilweise war eine Differenzierung zwischen den verschiedenen Fremdmaterialien jedoch nicht ausreichend möglich. Angaben zu der Differenzierung der unterschiedlichen Fremdmaterialien sind Kapitel 3.4. zu entnehmen.
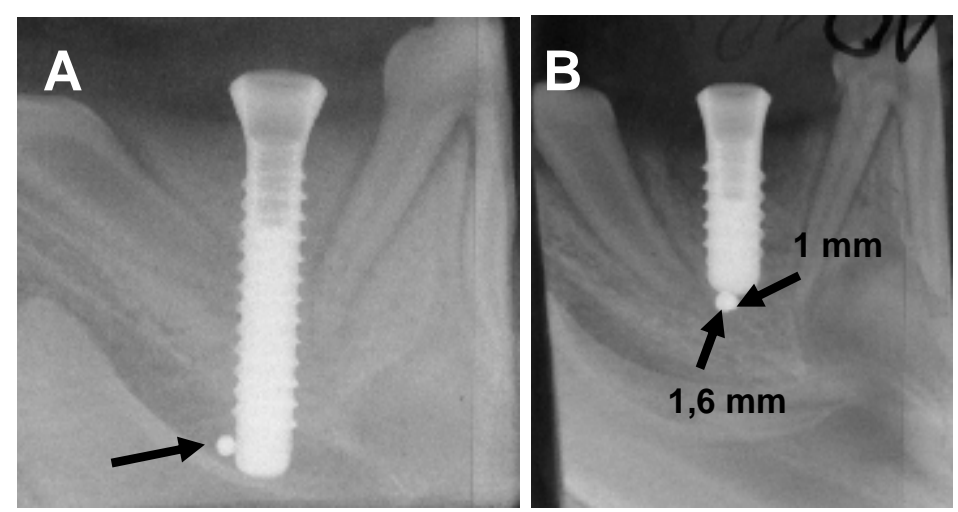

Abb. 28.1: Zahnfilm; Fremdmaterial Metallkugel (siehe Pfeil); $A$ : 1 Metallkugel $\varnothing 1$ mm (Präparat Nr. 2), B: 2 Metallkugeln unterschiedlicher Größe $\varnothing 1 \mathrm{~mm}$ und $\varnothing$ 1,6 mm (Präparat Nr. 10). 


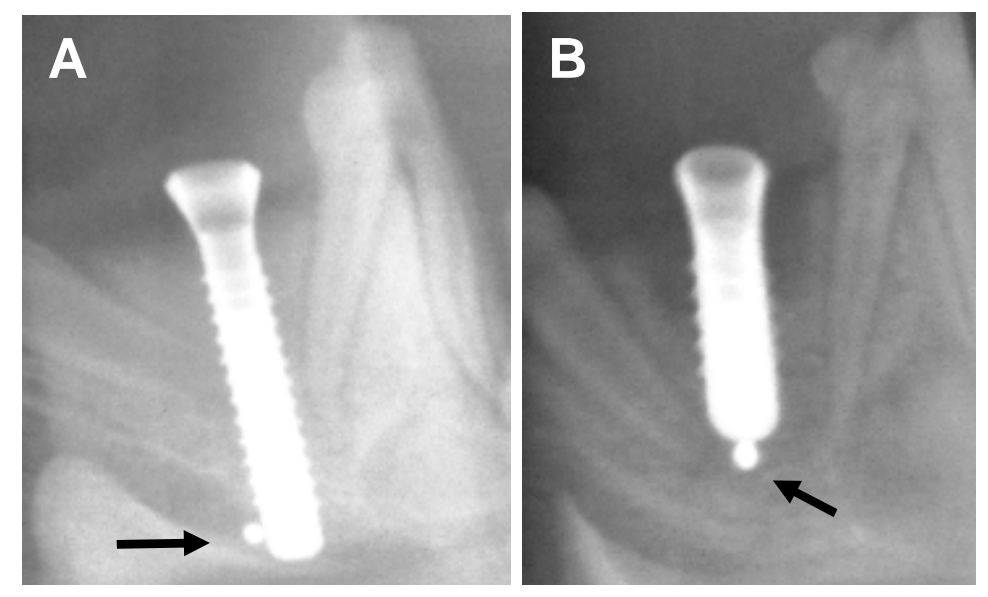

Abb. 28.2: OPAN; Fremdmaterial Metallkugel (siehe Pfeil); A: 1 Metallkugel $\varnothing 1 \mathrm{~mm}$ (Präparat Nr. 2), B: 2 Metallkugeln unterschiedlicher Größe $\varnothing 1 \mathrm{~mm}$ und $\varnothing 1,6 \mathrm{~mm}$ (Präparat Nr. 10).

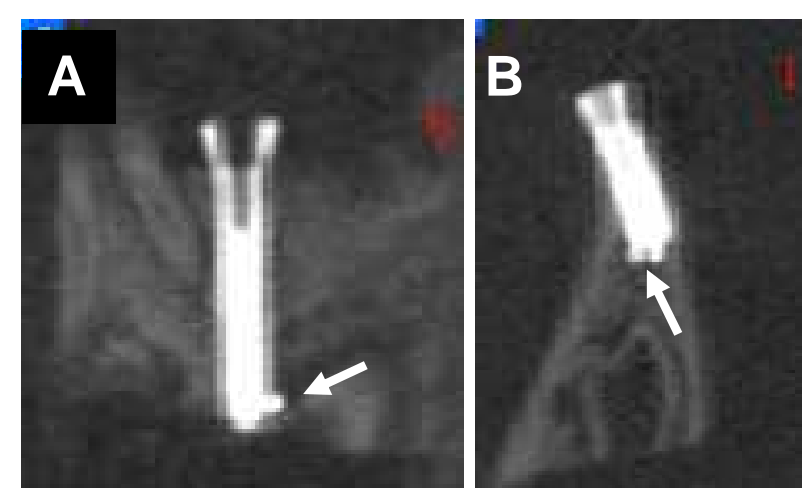

Abb. 28.3: DVT; Fremdmaterial Metallkugel (siehe Pfeil); A: sagittale Schicht, 1 Metallkugel Ø $1 \mathrm{~mm}$ (Präparat Nr. 2), B: koronare Schicht, 2 Metallkugeln unterschiedlicher Größe Ø $1 \mathrm{~mm}$ und $\varnothing 1,6 \mathrm{~mm}$ (Präparat Nr. 10). 

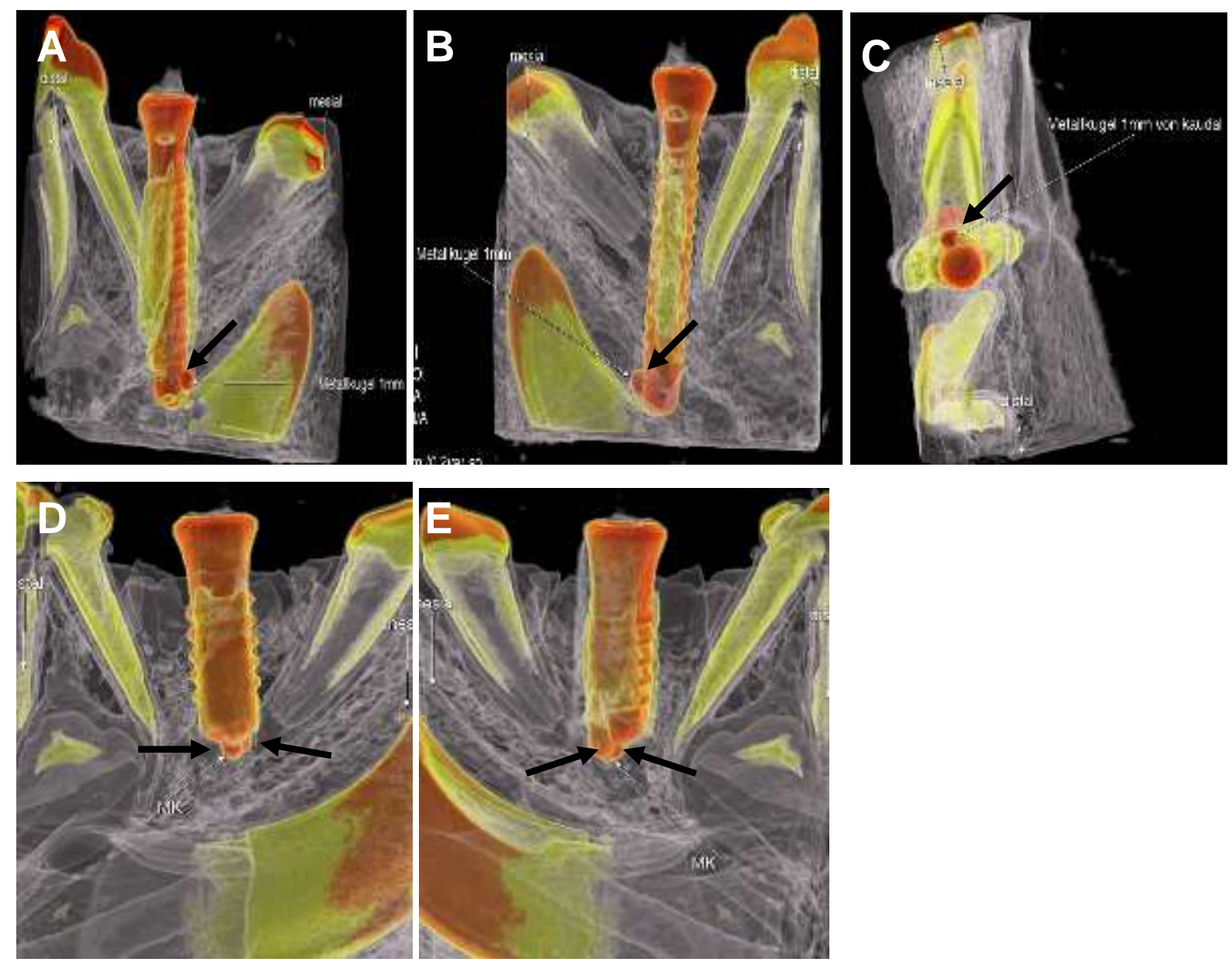

Abb. 28.4: fpVCT; Fremdmaterial Metallkugel (siehe Pfeile) VR-Technik, Transparentmodus I; A-C: 1 Metallkugel $\varnothing 1 \mathrm{~mm}$ (Präparat Nr. 2,) A: Ansicht von vestibulär, B: Ansicht von lingual, C: Ansicht von kaudal, D-E: 2 Metallkugeln unterschiedlicher Größe $\varnothing 1 \mathrm{~mm}$ und $\varnothing$ 1,6 mm (Präparat Nr. 10), D: Ansicht von vestibulär, E: Ansicht von lingual.

\section{Guttapercha:}

Bei vier Implantatschweinkieferproben (Präparate 3, 6, 12, 13) wurden

Guttaperchastifte als Fremdmaterial inseriert. In 26 von 48 möglichen Fällen konnte Fremdmaterial detektiert werden, in 14 dieser Fälle war eine genaue Differenzierung möglich. Dabei wurde in einer Prüfungssituation (Präparat 3) die Guttapercha anhand aller Verfahren fälschlicherweise als Metallkugel beschrieben. Nur mit der fpVCTMethode konnte an den drei weiteren Präparaten Guttapercha beobachtet und korrekt befundet werden (Abb. 29.1 und 29.2). 

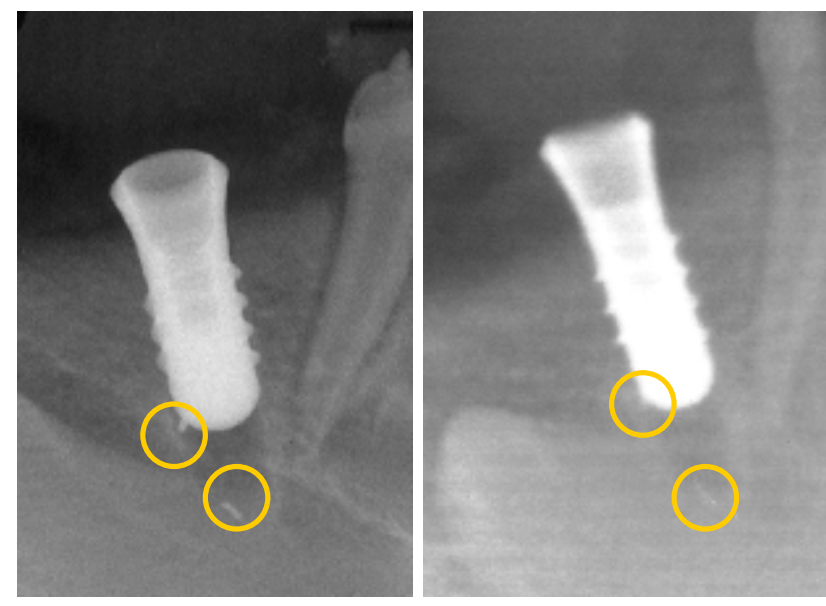

Abb. 29.1: Fremdmaterial Guttapercha: 2 Guttaperchastifte Länge $1 \mathrm{~mm}$ (siehe Kreise) Präparat Nr. 13; A: Zahnfilm, B: OPAN.
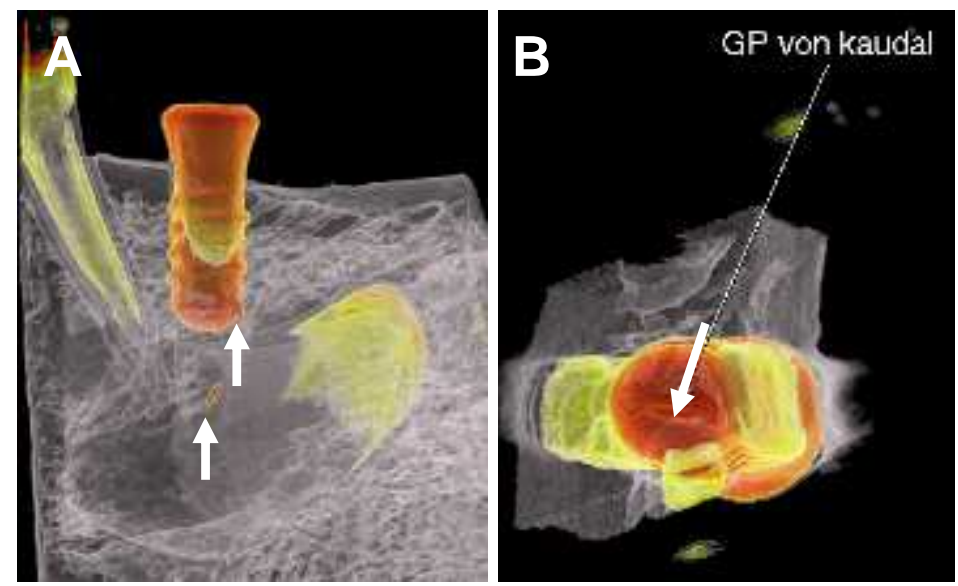

Abb. 29.2: Fremdmaterial Guttapercha fpVCT VR-Technik, Transparentmodus I; $\boldsymbol{A}$ : 2 Guttaperchastifte Länge 1 mm (siehe Pfeil) Präparat Nr. 13, B: 1 Guttaperchastift Länge $1 \mathrm{~mm}$ Ansicht von kaudal (siehe Pfeil) Präparat Nr. 6.

\section{B-TCP:}

Die Detektion von feingranulärem B-TCP war insgesamt nur sehr eingeschränkt möglich. Es zeigt eine ähnliche Röntgenopazität wie Knochengewebe. An einem von drei Präparaten (Probekörper 12) konnte das Granulat mit allen Verfahren gut beurteilt werden (Abb. 30.1 und 30.2). 


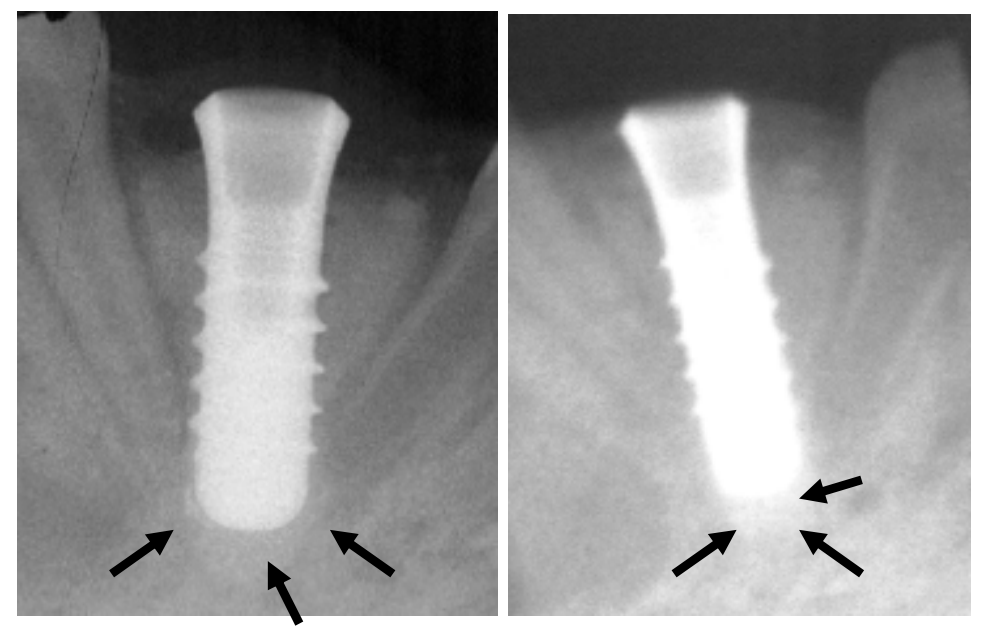

Abb. 30.1: Fremdmaterial B-TCP (siehe Pfeile) Präparat Nr. 12; A: Zahnfilm, B: OPAN.
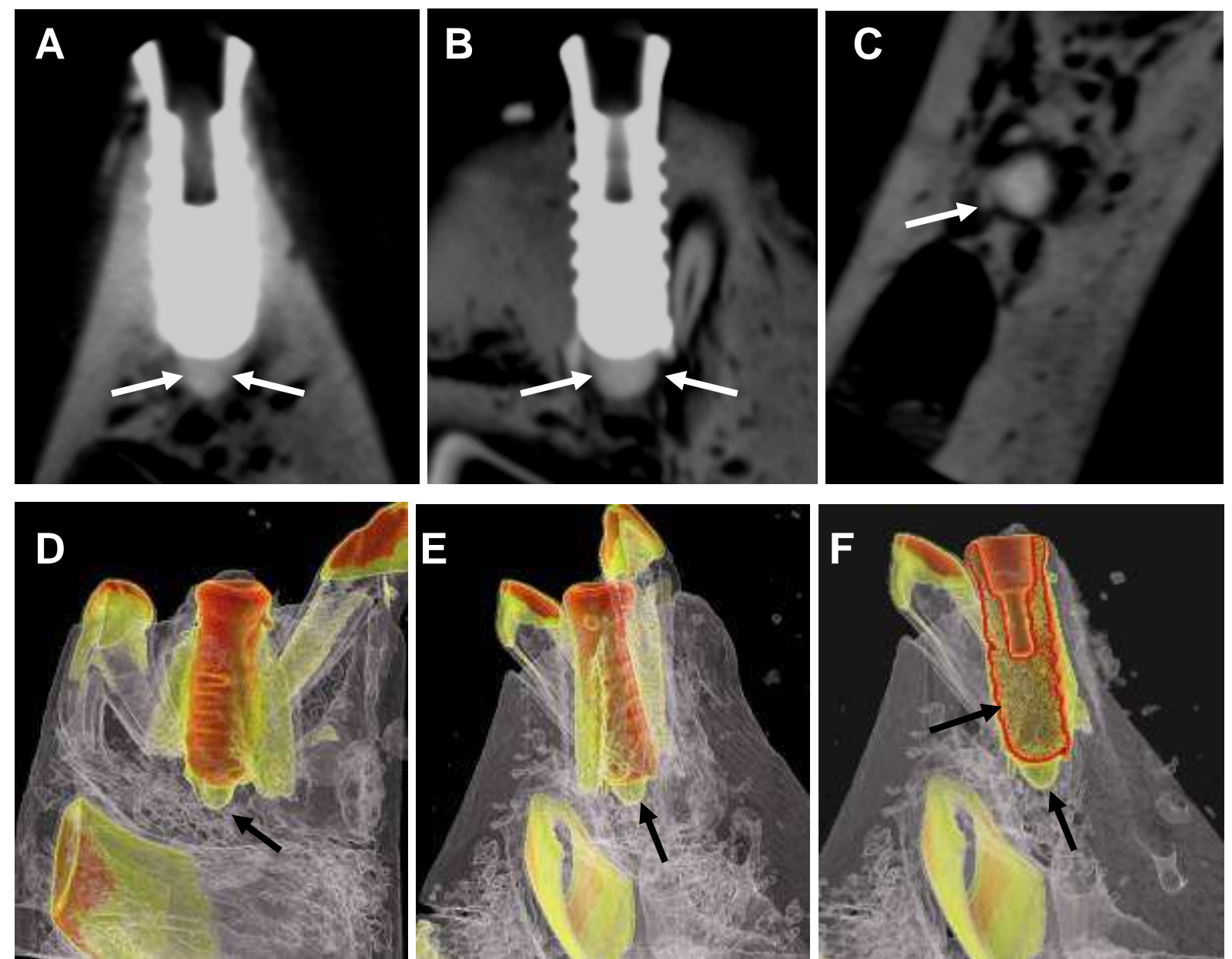

Abb. 30.2: fpVCT: Fremdmaterial B-TCP (siehe Pfeil) Präparat Nr. 12; A: koronare Schicht, B: sagittale Schicht, C: Ansicht von kaudal, D-E: VR-Technik, Transparentmodus I, D: Ansicht von vestibulär, E: Ansicht von distal, F: Ansicht von distal, „Front-Cut-Modus“ mit Schnittbild durch das Innere des Implantates (rote Linie markiert Außenseite des Implantates). 


\subsubsection{Beeinträchtigung der Bildauswertung durch Streustrahlenartefakte}

Sowohl bei den Zahnfilmaufnahmen als auch bei den Panoramaschichtaufnahmen traten in keinem Fall Streustrahlenartefakte auf, die zu einer Beeinträchtigungen der Bildauswertung hätten führen können.

Sowohl bei den DVT-Daten als auch bei den fpVCT-Daten kam es zu störenden Artefakten (Abb. 31). Bei den DVT-Aufnahmen zeigten sich in 35,71 \% der Fälle Streustrahlenartefakte, die in $26,20 \%$ auch zu einer Beeinträchtigung der Bildauswertung geführt haben.

Bei der fpVCT kam es in 78,57 \% zu Streustrahlenartefakten, die die Bildauswertung in $57,14 \%$ negativ beeinflussten.
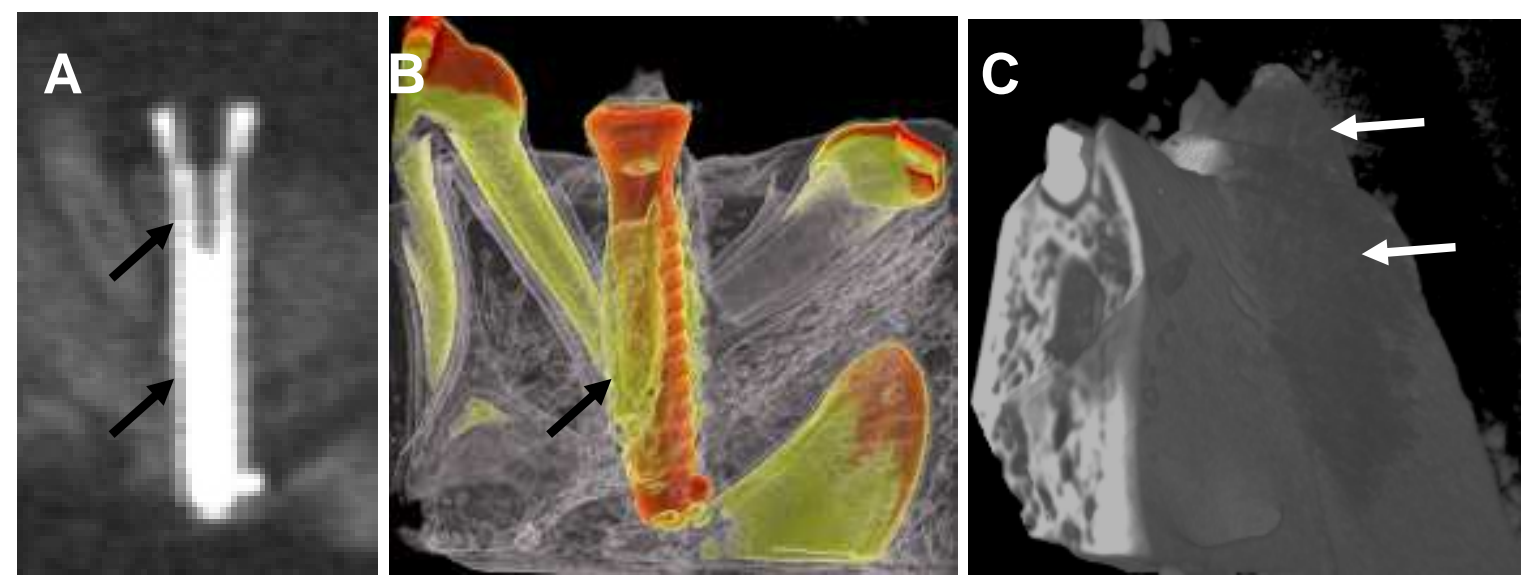

Abb. 31: Streustrahlenartefakte (siehe Pfeil) Beispiel Präparat Nr. 2; A: DVT, B: fpVCT VR-Technik Transparentmodus I Ansicht von vestibulär, C: fpVCT 3DRekonstruktion.

\subsection{Zweidimensionale versus dreidimensionale Röntgenverfahren}

Im Folgenden sind die konventionellen zweidimensionalen Röntgenverfahren Zahnfilm und OPAN zusammengefasst den dreidimensionalen Verfahren DVT und fpVCT gegenübergestellt. Es wurde die Detektierbarkeit der Defekte an Knochen und Implantaten sowie von Fremdkörpern evaluiert. 


\section{Alveolardefekt:}

Es sind sowohl mit den zweidimensionalen als auch mit den dreidimensionalen Darstellungsverfahren vergleichbare Aussagen bzgl. der Darstellung von Alveolardefekten möglich (Abb. 32). Es zeigt sich, dass bei der Beurteilung von intaktem Knochengewebe häufiger korrekte Angaben getroffen werden konnten als bei Vorliegen eines Alveolardefektes.

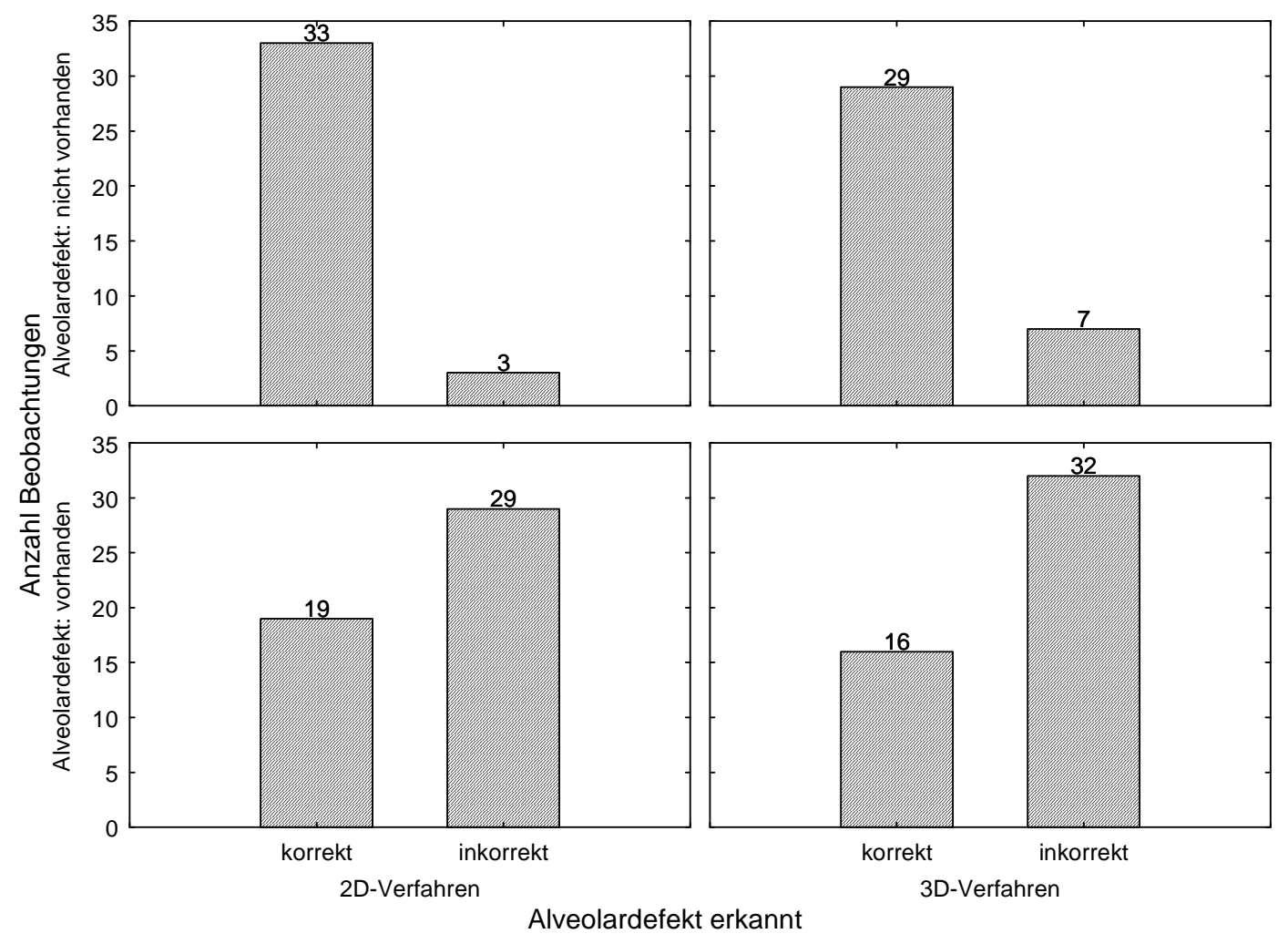

Abb. 32: Korrekte bzw. falsche Beurteilung des Vorhandenseins bzw.

Nichtvorhandenseins von Alveolardefekten (unterschiedliche Defektmorphologien zusammengefasst).

Implantatdefekt:

Hierbei zeigt sich kein wesentlicher Unterschied zwischen den 2D- und 3D-Verfahren (Abb. 33). Deutlich wird aber die in hohem Maße genauere Beurteilung von intakten Implantaten im Vergleich zu den iatrogen beschädigten Implantaten. Bei der Erkennung von Implantatdefekten überwiegen in beiden Verfahrenskategorien die inkorrekten Aussagen. 


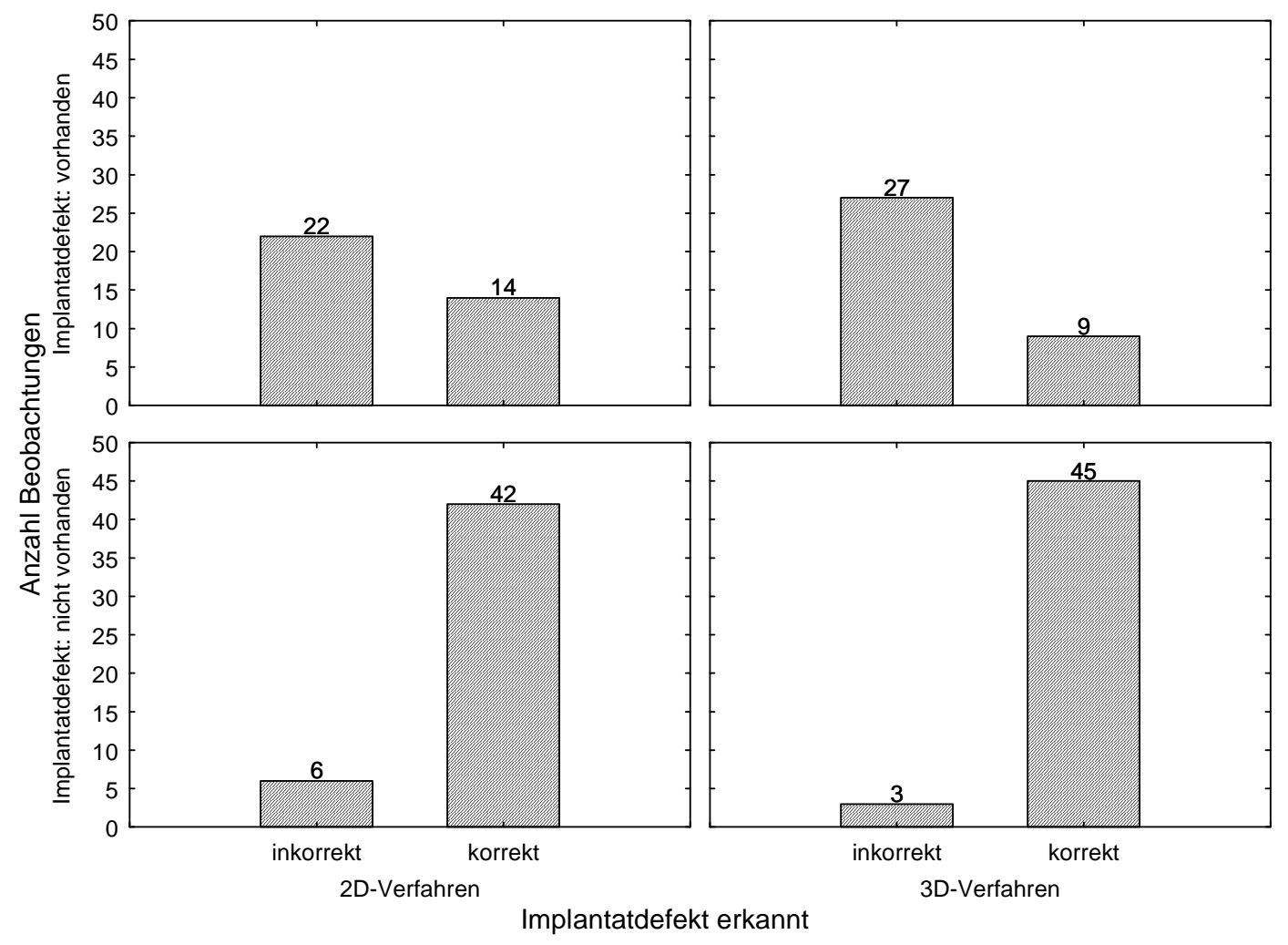

Abb. 33: Korrekte bzw. falsche Beurteilung des Vorhandenseins bzw.

Nichtvorhandenseins von Implantatdefekten (unterschiedliche Arten der Defekte zusammengefasst).

\section{Fremdkörper:}

Deutlich zu erkennen ist der überwiegende Anteil an korrekten Aussagen zum Vorliegen bzw. Fehlen einer oder mehrerer Fremdkörper (Abb. 34). Dabei unterscheiden sich die beiden gegenübergestellten Röntgenverfahren nur unwesentlich voneinander. 


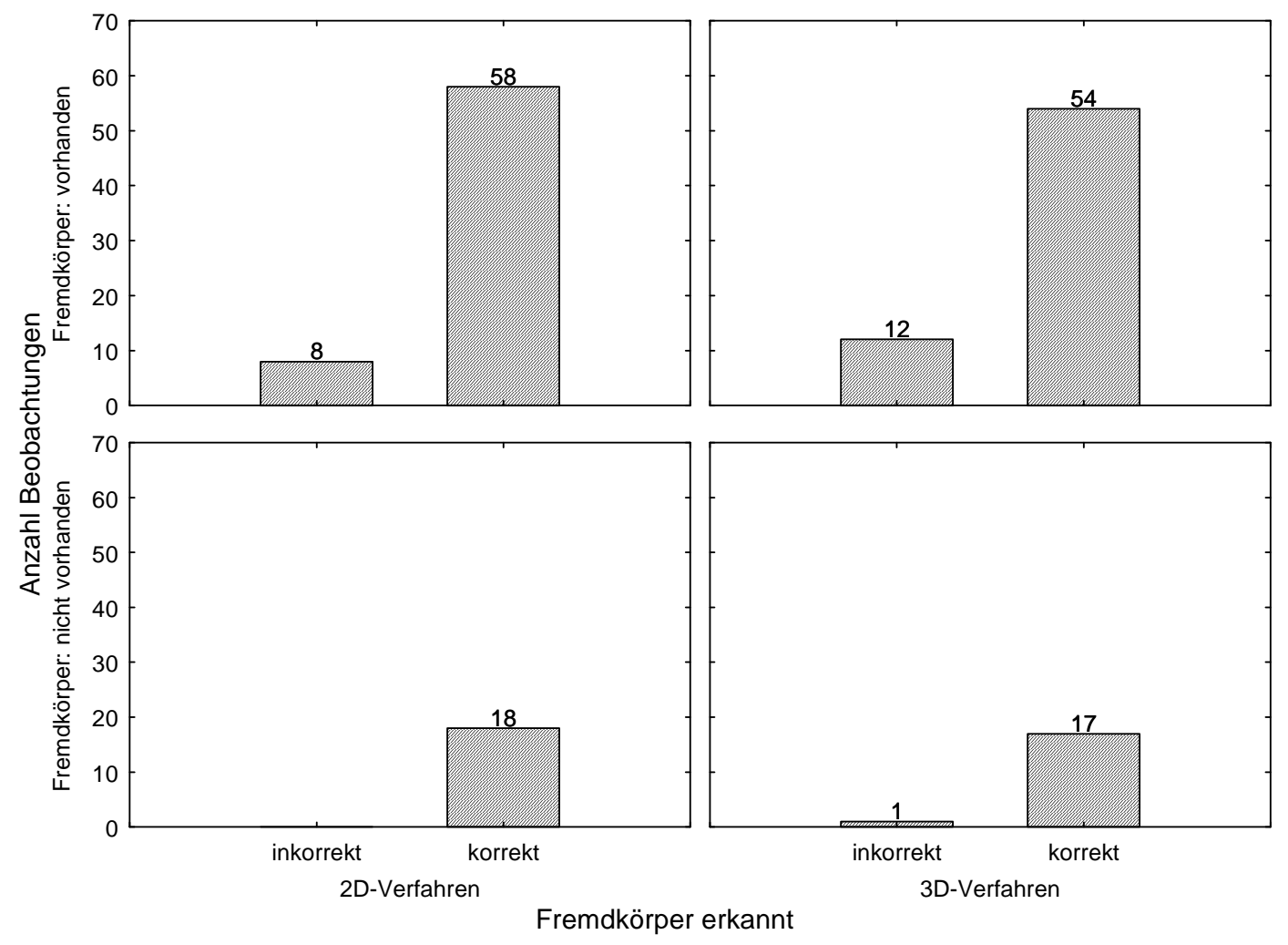

Abb. 34: Korrekte bzw. fehlerhafte Befundung des Vorhandenseins bzw. Fehlens von Fremdkörpern (unterschiedliche Fremdkörper-Typen zusammengefasst).

\subsection{Differenzierte Analyse der Fremdkörperdetektion}

Bei der bisherigen Evaluation der Fremdkörper wurde nur untersucht, ob die Situation „Fremdkörper ja/nein“ korrekt erkannt wurde. Im Folgenden wurde zusätzlich überprüft, ob das Material des Fremdkörpers ( $T=$ B-TCP-Keramik, MK= Metallkugel, $\mathrm{GP}=$ Guttaperchastift) richtig klassifiziert wurde. Dabei gingen nur diejenigen Schweinekieferproben in die Analyse mit ein, bei denen auch ein Fremdkörper inseriert war. Anhand dieser Untersuchung wurden sowohl die Röntgenmethode als auch die Untersucher im Sinne einer deskriptiven Analyse verglichen. 


\subsubsection{Fremdkörperaufdeckung: Vergleich der Röntgenmethoden}

Der Abbildung 35 ist zu entnehmen, wie häufig mit den einzelnen Röntgenmethoden eine korrekte Zuordnung der Werkstoffe möglich war.

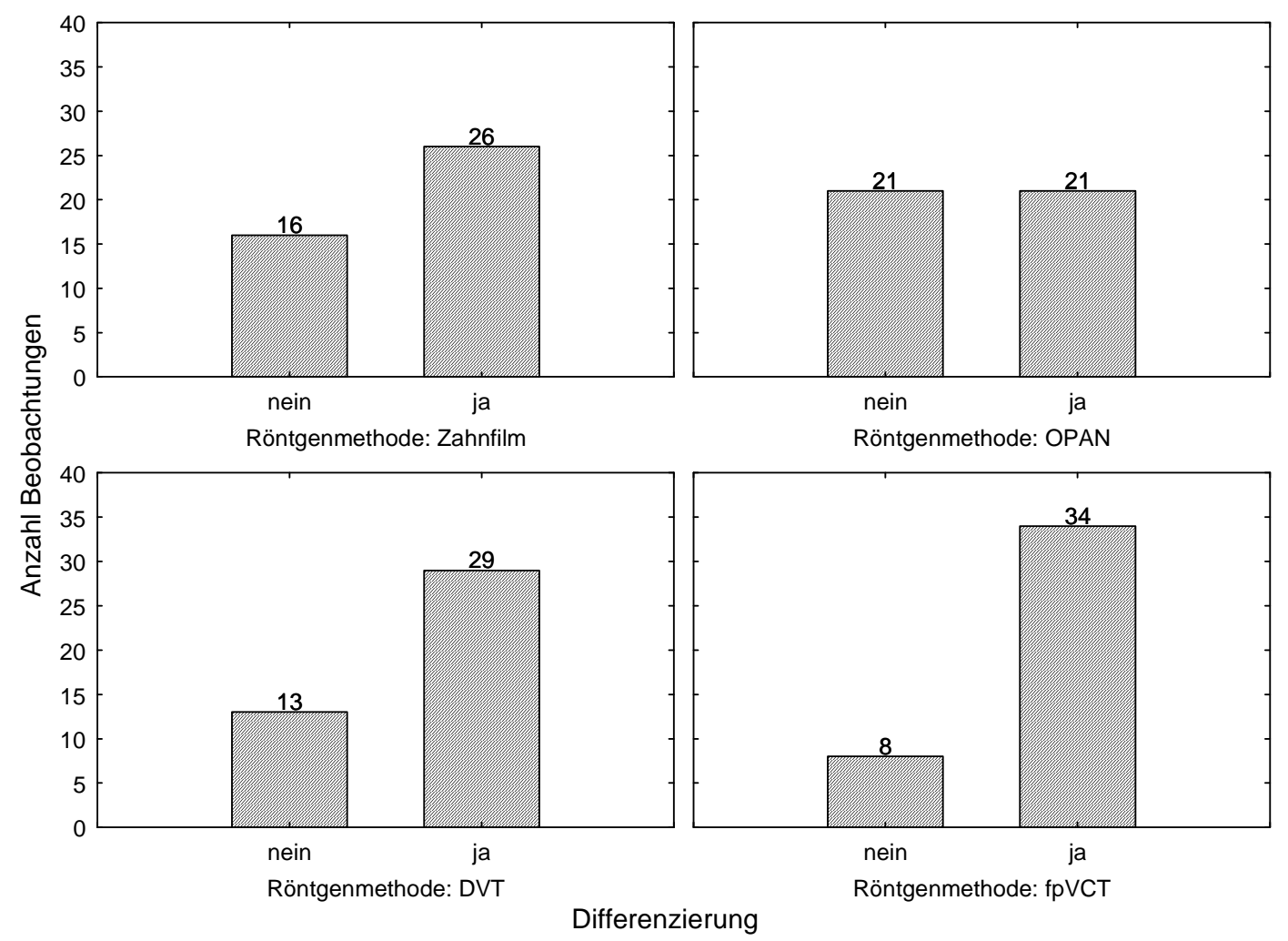

Abb. 35: Differenzierbarkeit der iatrogen inserierten Fremdkörper mit den verschiedenen Röntgenverfahren (ja= richtig beurteilt, nein= falsch beurteilt).

Die fpVCT ermöglichte im Vergleich zu den übrigen Methoden eine deutlich höhere Anzahl an richtigen Befunden hinsichtlich der Beurteilung der verschiedenen Werkstoffe. Außerdem ist erkennbar, dass die dreidimensionalen Röntgenverfahren fpVCT $(81 \%)$ und DVT (69\%) den zweidimensionalen Verfahren OPAN $(50 \%)$ und Zahnfilm (61\%) bezüglich einer korrekten Differenzierung des Fremdmaterials überlegen sind.

Nachfolgend sind die Vergleiche entsprechend den unterschiedlichen Fremdkörpern aufgeführt (Abb. 36). Die Methoden unterscheiden sich hierbei nur unwesentlich. Es lässt sich aber erkennen, dass Fremdkörper vom Typ Metallkugel mit allen Methoden 
leichter korrekt erkannt wurden als die anderen zwei Fremdkörper-Typen. Zudem konnte Guttapercha im DVT überhaupt nicht detektiert werden.

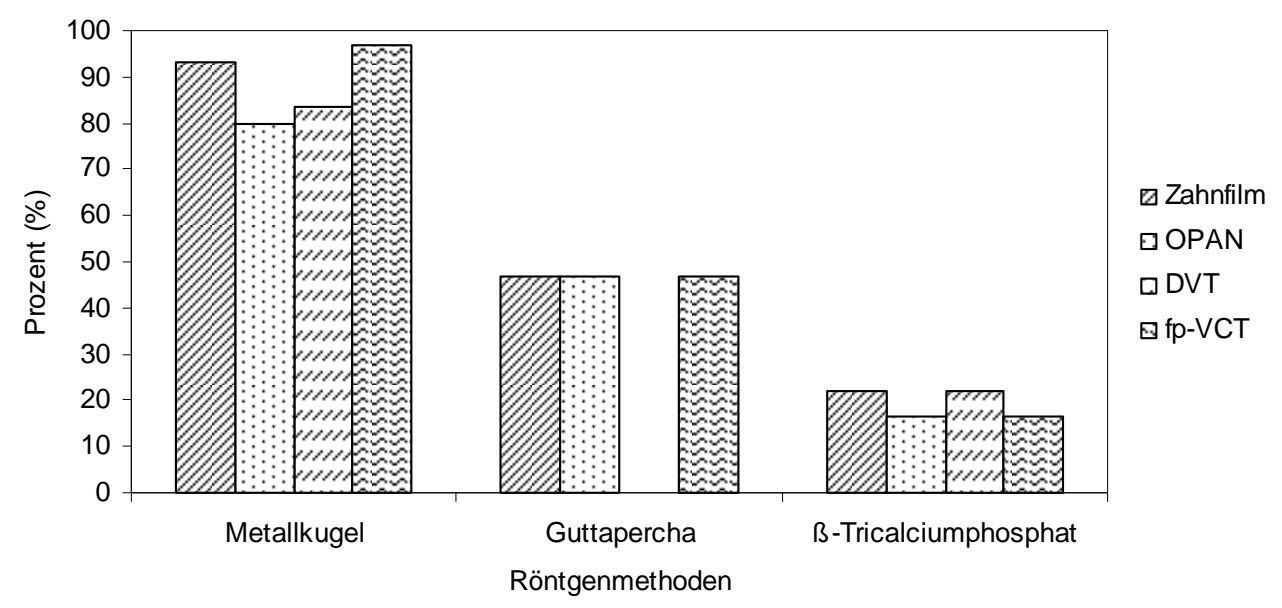

Abb. 36: Vergleich der Röntgenverfahren: Häufigkeit korrekt erkannter Fremdmaterialien.

\subsection{Vergleich der Untersucher}

Der Einfluss des Untersuchers auf die erhobenen Befunde wurde hinsichtlich der Angaben zu Messungen von Implantatlänge und -durchmesser und der Beurteilung von Knochen- und Implantatdefekten sowie Fremdkörpern verglichen. Die Präzision der drei Untersucher bei der Befundung wurde mit demselben statistischen Verfahren (F-Test in einer nichtparametrischen Varianzanalyse) wie im Vergleich der Röntgenmethoden analysiert und die Ergebnisse anschließend prozentual visualisiert.

Bei keinem Test konnte ein signifikanter Unterschied zwischen den Untersuchern festgestellt werden (Abb. 37). Es liegen folgende $p$-Werte für die einzelnen Testreihen vor: Implantat-Längenmessung: 0,7663; Implantat-Durchmessermessung: 0,3876; Knochendefekte: 0,4578; Implantatdefekte: 0,2425; Fremdkörper: 0,8068. Ein Unterschied zwischen dem Radiologen und den beiden Zahnmedizinern bestand ebenfalls nicht. 


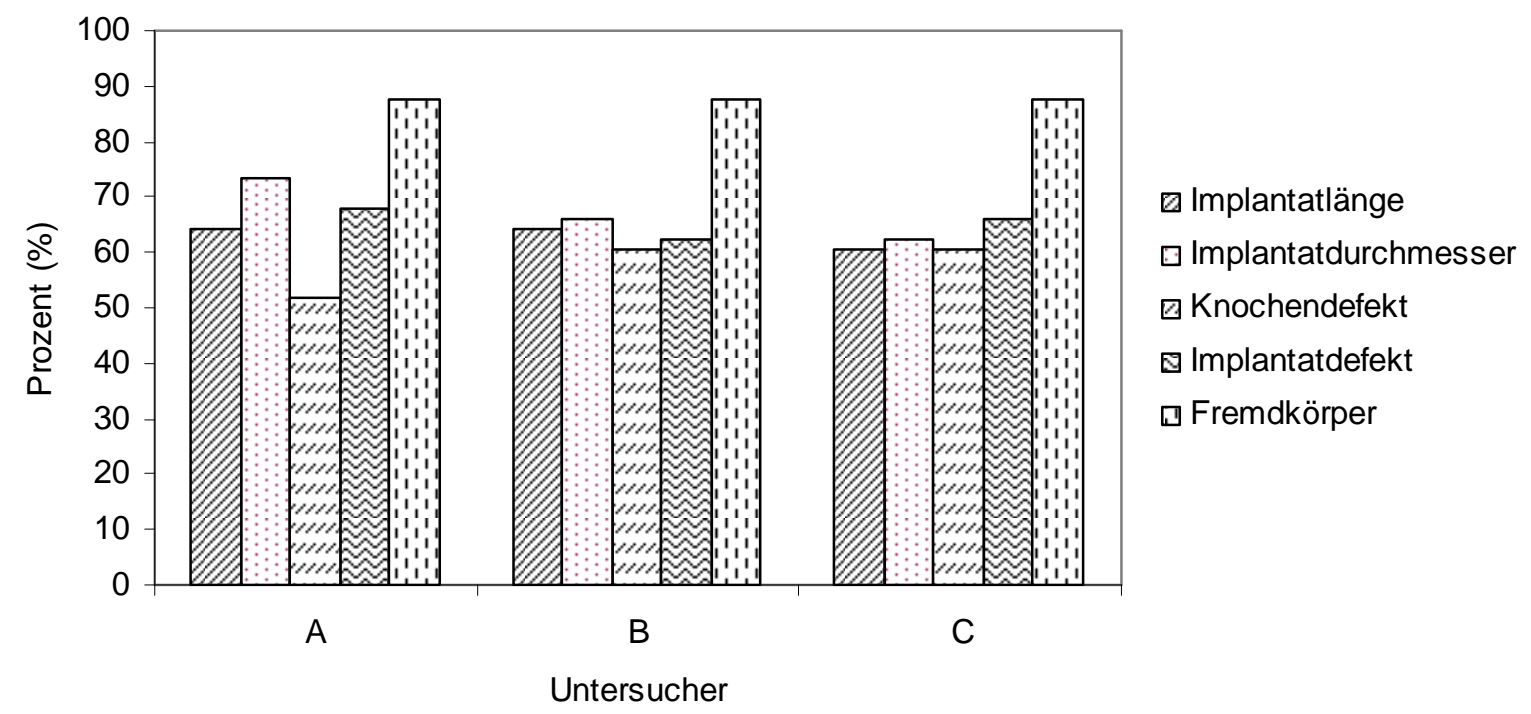

Abb. 37: Vergleichende Darstellung: Häufigkeit korrekt gemessener Implantatlängen und-durchmesser bzw. des korrekt festgestellten Vorhandenseins eines Knochen-, eines Implantatdefektes und eines Fremdkörpers durch Untersucher A (Radiologe), $B$ und $C$ (Oralchirurgen).

Entsprechend des zuvor erwähnten Verfahrens wurden die Untersucher bezüglich der Fremdkörperdetektion verglichen (Abb. 38). Es zeigten sich keine wesentlichen Unterschiede zwischen den Untersuchern. Deutlich wird jedoch, dass Fremdmaterial in Form einer Metallkugel deutlich häufiger richtig beurteilt werden konnte als die anderen beiden Fremdmaterialien.

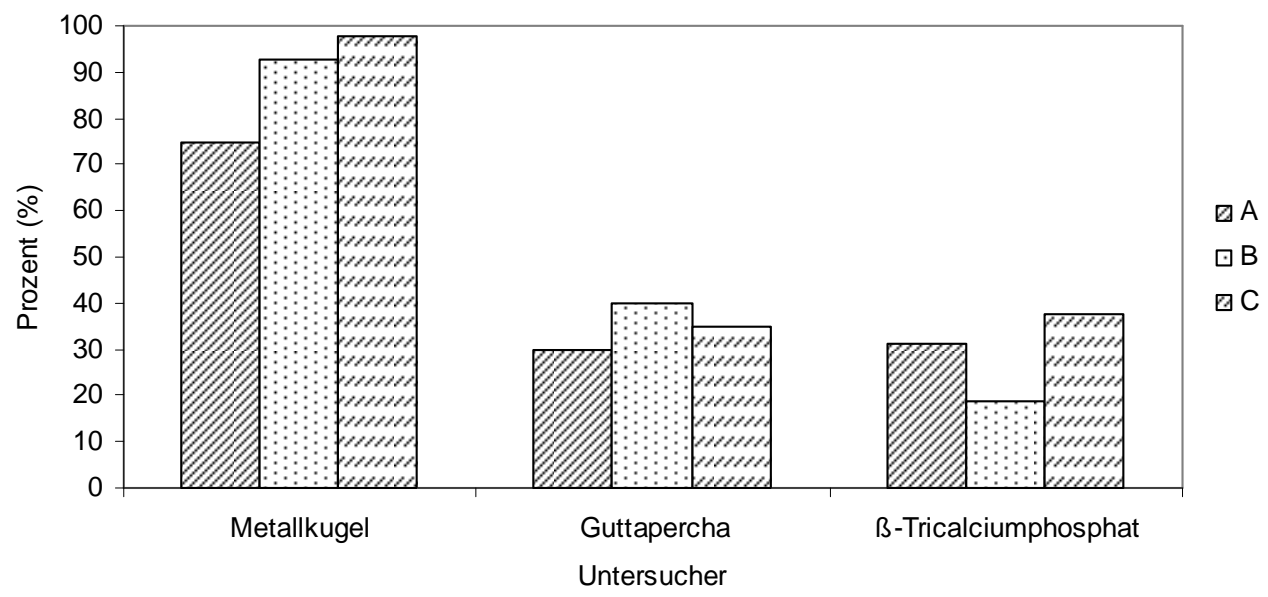

Abb. 38: Vergleich der Untersucher ( $A=$ Radiologe, $B, C=$ Oralchirurgen) anhand der Häufigkeit korrekt beurteilter Fremdkörper. 
Außerdem wurde die Verteilung der Messabweichungen zu den Originallängen und Originaldurchmessern bei der Längen- bzw. Durchmesserbestimmung getrennt für die einzelnen Untersucher evaluiert (Abb. 39 A-B).

$\boldsymbol{A}$

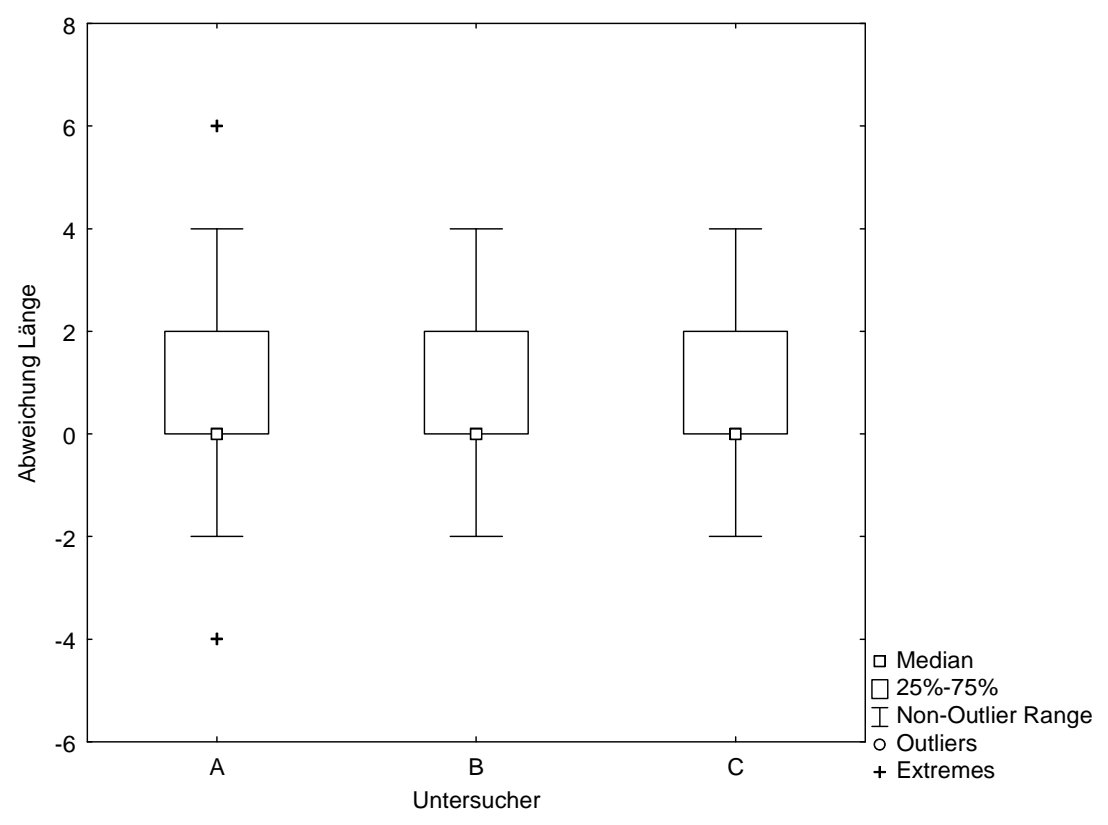

B

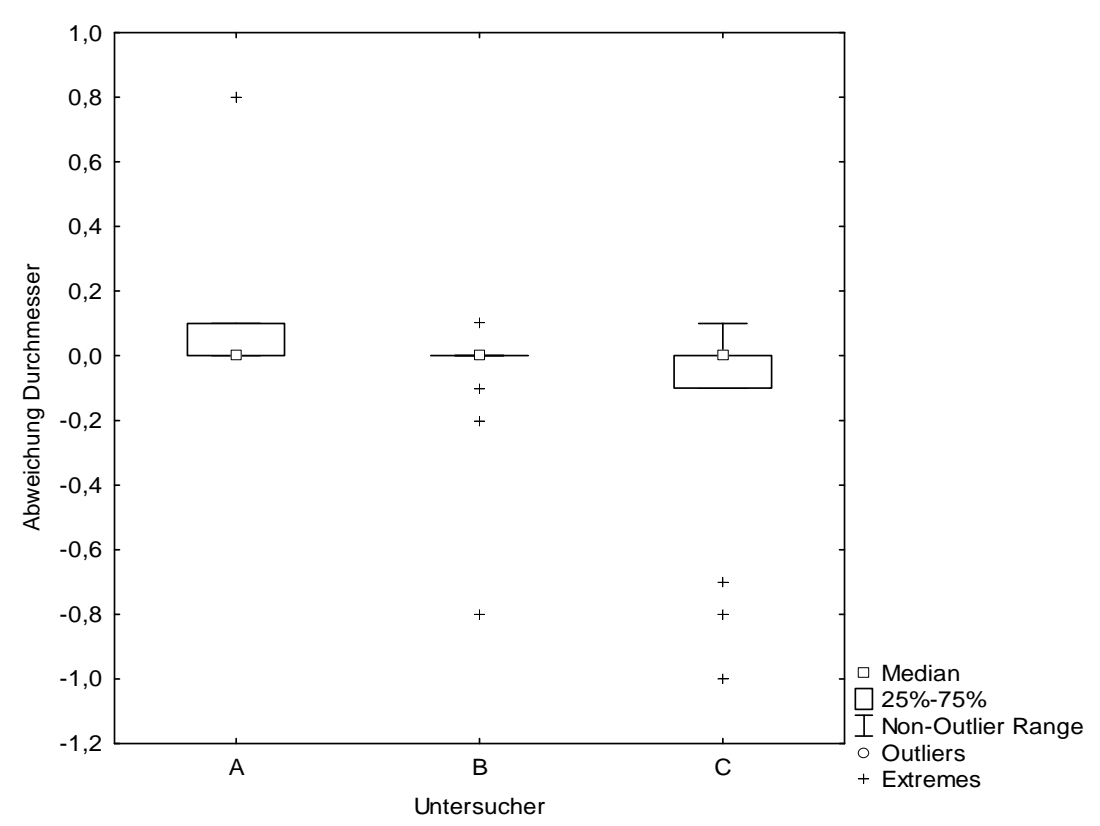

Abb. 39: Bestimmung A: Implantatlängen und B: Implantatdurchmesser im Vergleich der 3 Untersucher ( $A=$ Radiologe, $B, C=$ Oralchirurgen), Abweichungen von der Originallänge bzw. dem Originaldurchmesser. 
Der Median liegt bei allen Untersuchern bei der fehlerfreien Messung der Implantate. Bei den Messungen der Implantatlängen zeigen sich bzgl. der Abweichungen zu großer bzw. zu kleiner Messangaben keine Unterschiede zwischen den Untersuchern. 


\section{Diskussion}

Das Ziel dieser Untersuchung war die Evaluation der Abbildungsgenauigkeit verschiedener zweidimensionaler und dreidimensionaler radiologischer Darstellungsverfahren bei der Insertion dentaler Implantate und der Beurteilung periimplantärer Strukturen anhand von Schweinekiefer-Modellen. Insbesondere sollte dabei überprüft werden, inwieweit die derzeit präklinisch einsetzbare fpVCTTechnologie verzerrungs- und zerstörungsfreie Bilddaten von Hochkontraststrukturen liefert und damit für die Implantationsdiagnostik besser geeignet wäre als bereits klinisch verfügbare Röntgenverfahren.

Die Bilddaten der verschiedenen Verfahren wurden hinsichtlich der Qualität ihrer Ortsauflösung und Detailgenauigkeit überprüft und zudem auf ihre Darstellung von dreidimensionalen Hochkontraststrukturen untersucht.

Eine wesentliche Voraussetzung für eine erfolgreiche Implantatinsertion sind neben der Detektion des N. alveolaris inferior sowohl die quantitative als auch die qualitative Bestimmung des Knochenangebotes, das Erkennen pathologischer Prozesse, die Inklination des Alveolarfortsatzes und die lokalen Verhältnisse der anatomischen Strukturen in Relation zum Ort der geplanten Insertion (Frederiksen 1995, Gher und Richardson 1995, Molly 2006, Fuh et al. 2010). Um sowohl präimplantologisch als auch im Behandlungsverlauf suffiziente Aussagen hinsichtlich des Implantationserfolges treffen zu können, ist weiterhin eine wesentliche Voraussetzung, dreidimensionale Strukturen, die sich häufig auch im Mikrometerbereich befinden, mit einer hohen isotropen Ortsauflösung und verzerrungsfrei darstellen zu können (Garg und Vicari 1995, Greenstein und Tarnow 2006, Mengel et al. 2006, Peker et al. 2008, Vandenberghe et al. 2008). Im Rahmen der Diagnostik werden im Klinikalltag neben der klinischen Untersuchung bereits Röntgenbilder der interessierenden Region angefertigt. Die hier angewandten Verfahren wie Zahnfilm (ZF), Orthopantomograph (OPG) und digitaler Volumentomograph (DVT) finden derzeit bereits im Rahmen von Routineuntersuchungen der Mund-, Kiefer- und Gesichtschirurgie regelmäßig Anwendung.

Für die zerstörungsfreie dreidimensionale Darstellung in zahnanatomischen wissenschaftlichen Untersuchungen wurden bisher Mikro-CT-Systeme eingesetzt. 
Hiermit können endodontische Strukturen experimentell mit einer Ortsauflösung von wenigen Mikrometern (Engelke et al. 1999, Rhodes et al. 2000, Peters et al. 2003) bzw. $13 \mathrm{LP} / \mathrm{mm}$ (Peters et al. 2000, Peters et al. 2003) dargestellt werden. Der in dieser Studie angewandte Prototyp mit fpVCT-Technologie ermöglicht im Vergleich dazu eine Ortsauflösung von 3,6 LP/mm (140 $\mu \mathrm{m})$. MSCTs haben mit 1,7 LP/mm eine noch etwas geringere Auflösung und liefern meist keine isotropen Daten. Der intraorale ZF hingegen erreicht eine Ortsauflösung von $10-12 \mathrm{LP} / \mathrm{mm}$. Durch MikroCT-Systeme können jedoch aufgrund ihrer Bauweise nur wenige Objekte mit einer geringen Größe untersucht werden, wie z.B. einzelne Zähne (Peters et al. 2000, Peters et al. 2003), so dass der Gantry des hier angewandten fpVCT mit einer Gantryöffnung von $43,8 \mathrm{~cm}$ im Durchmesser ein klarer Vorteil für die präklinische Diagnostik zugesprochen werden kann. Untersuchungen größerer Objekte wie z.B. des menschlichen Schädels werden damit durchführbar (Heidrich et al. 2005 a). Allerdings können die Untersuchungszeiten der Mikro-CT bis zu 6 Stunden andauern, da eine Verbesserung der Ortsauflösung mit einer verlängerten Scanzeit einhergeht (Peters et al. 2000, Peters et al. 2001, Peters et al. 2003). Die fpVCT hingegen benötigt Untersuchungszeiten von weniger als 10 Sekunden infolge der neuen Detektortechnologie (Ning et al. 2000, Ning et al. 2003, Kiessling et al. 2004, Hannig et al. 2005, Heidrich et al. 2005 b).

Weitere Vorteile für die fpVCT ergeben sich aufgrund der physikalischen Gegebenheiten. Neben der hohen isotropen Ortsauflösung haben die Flachbilddetektoren, insbesondere die mit indirekter Konversion von Röntgenstrahlen auf der Basis von Szintillatoren aus Cäsiumjodid (CsJ), im Vergleich zu heute etablierten Bildverstärkern über den gesamten Ortsfrequenzbereich höhere DQEWerte („detective quantum efficiency“) im Bereich von 50\% (Spahn et al. 2003). Die DQE beschreibt dabei die Fähigkeit eines Detektors, die auf das Eintrittsfenster auftreffende Röntgenstrahlung bildwirksam umzusetzen (Spahn et al. 2003). Verglichen mit der DQE der MSCT von mehr als $90 \%$ zeigen sie jedoch eine geringere Quantenausbeute (Kalender und Kyriakou 2007, Hahn et al. 2009 a, Kyriakou et al. 2009) und sind somit weniger effizient, so dass sie bei gleicher Dosis erwartungsgemäß ein höheres Rauschen und eine niedrigere Niedrigkontrastauflösung produzieren (Kyriakou et al. 2009). Das Signalnachleuchten für radiographische Darstellungen ist unbedeutsam (Kalender 2003). Es konnte gezeigt werden, dass die fpVCT einen geringen 
Bildverlust, d.h. ein geringes Nachleuchten (image lag $<1 \%$ ) und eine hervorragende Linearität ( $1 \%$ ) aufweist. Geometrische Verzerrungen treten kaum auf. Daneben wird eine schnelle Bildfolgesequenz ermöglicht (Ning et al. 2000). Diese Technologie bietet beste Vorraussetzungen für die hochauflösende Darstellung von Hochkontrastobjekten (Kalender 2003) und erreicht zudem eine weitaus bessere räumliche Auflösung als Standard-CT-Systeme. Kalender postulierte, dass bei einer Steigerung der Ortsauflösung zu berücksichtigen ist, dass die Varianz des Bildpunktrauschens bei unveränderten Scan- und Rekonstruktionsparametern und unverändertem Objektdurchmesser mit der 4. Potenz des Abtastabstandes und damit umgekehrt proportional zur 4. Potenz der Ortsauflösung ansteigt (Kalender 2003). So kann die Weichgewebe- oder Niedrigkontrastauflösung nicht in gleicher Weise gesteigert werden wie die Ortsauflösung, ohne dabei einen erheblichen Dosisanstieg in Kauf zu nehmen. Je nach Kontrast des darzustellenden Objektes können Daten mit einer Ortsauflösung von $150 \mu \mathrm{m}$ (Hochkontrastobjekte) bis zu $250 \mu \mathrm{m}$ (Niedrigkontrastobjekte) erzielt werden (Hermann et al. 2005).

In der MSCT können je nach Gerät Auflösungen in der z-Richtung von $300 \mu \mathrm{m}$ bis $700 \mu \mathrm{m}$ erreicht werden (Kalender 2003), wobei jedoch die Auflösung innerhalb der Schicht kleiner als die Schichtdicke ist (Hermann et al. 2005). Verminderte Auflösungen in der z-Richtung führen zur Bildung von Stufenartefakten. Durch die verglichen mit dem fpVCT schlechtere Auflösung ist auch der Einfluss durch Partialvolumeneffekte auf das Bild erhöht und somit eine schlechtere Abgrenzung benachbarter Gewebe und eine reduzierte Erkennbarkeit kleinster Strukturen gegeben (Kalender et al. 1994, Neff et al. 1998). Isotrope Voxel und die hohe Auflösung der fpVCT, welche aus der geringen Detektorelementgröße $(200 \times 200$ $\mu \mathrm{m})$, dem Vergrößerungsfaktor $(1,43)$ und der kleinen Brennfleckgröße der Röntgenröhre resultieren, reduzieren im Gegenzug dazu das Auftreten von Partialvolumeneffekten. Strukturen, die jedoch kleiner als die Voxelgröße sind, lassen sich auch in der fpVCT nicht ausreichend beurteilen.

Demgegenüber ist die DVT bereits heute im klinischen Einsatz der Zahnmedizin von großer Relevanz. Durch diese Technologie wird die Darstellung kleinster Strukturen mit einer Auflösung von $125 \mu \mathrm{m}$ bzw. 2-3 LP/mm (3D Accuitomo) ermöglicht, was mit der fpVCT vergleichbar ist. Die effektive Strahlendosis ist dabei vergleichbar mit der einer Panoramaschichtaufnahme (Arai et al. 2001). Weiterer Vorteil der CBCT- 
Technologie ist die hohe Qualität der Darstellung (Mozzo et al. 1998) mit einer zur MSCT-Technologie vergleichbar guten Abbildungsgenauigkeit (Kobayashi et al. 2004, Marmulla et al. 2005, Loubele et al. 2007). Die in zweidimensionalen Verfahren (OPG und ZF) projektionsbedingt auftretenden Überlagerungen und Verzerrungen können durch die CBCT-Technik vermieden werden (Mah und Hatcher 2004). Im Rahmen dieser Studie wurden neben Dentalimplantaten periimplantäre Hochkontraststrukturen untersucht. In Anlehnung an die Tatsache, dass periimplantäre Defekte im Rahmen des implantologischen Langzeiterfolges eine wesentliche Rolle spielen, wurden die Knochendefekte als strukturelle Veränderung des knöchernen Angebotes gesetzt. Die durchzuführenden postimplantologischen Kontrollaufnahmen der Insertionsregion dienen unter anderem dem Ausschluss von Implantatdefekten und vorliegenden Fremdkörpern. Die Implantat- und Alveolardefekte wurden dabei bewusst gesetzt, um Qualitätsunterschiede im Vergleich der befundeten Darstellungen durch die verschiedenen klinisch anwendbaren Röntgenverfahren (ZF, OPG, DVT) und der fpVCT explorieren zu können.

Ein objektiver Vergleich der einzelnen Verfahren ist auf Grund der unterschiedlichen Techniken und Parameter wie Ortsauflösung, Detailgenauigkeit und die Möglichkeit der 3D-Darstellung nicht möglich. Aus diesem Grund wurde ein Fragebogen erstellt, um diese Kriterien der klinischen Situation angepasst visuell beurteilen und anhand der Ergebnisse die Verfahren vergleichen zu können.

Für die Implantologie sind metrische Messungen notwendig, deren Genauigkeit von der tatsächlich erreichten Ortsauflösung, der Kontrastauflösung und dem SignalRauschverhältnis abhängig ist. Auch die Identifikation der Messpunkte hat entscheidenden Einfluss auf die Messgenauigkeit (DGZMK).

Zunächst wurden anhand von Distanzmessungen (Implantatdurchmesser und -länge) die Verfahren verglichen, wobei mit allen Methoden annähernd gleich gute Ergebnisse erzielt werden konnten. Mit den zweidimensionalen konventionellen Verfahren sind Messungen in der vertikalen Ebene ausreichend möglich, was mit den Ergebnissen von Batenburg et al. übereinstimmt (Batenburg et al. 1997). Es zeigte sich lediglich ein Unterschied der anhand von OPG-Aufnahmen durchgeführten Längenmessungen gegenüber den anderen Verfahren. Dies ist darauf zurückzuführen, dass die OPG einen Verzerrungsfehler aufweist, der bei einer exakten Evaluation der Daten berechnet werden muss (Buser et al. 1990, Tal und 
Moses 1991, Hollender 1992, Reddy et al. 1994, Frederiksen 1995). In Abhängigkeit von der Entfernung zur Film- oder Sensorebene ergeben sich Vergrößerungen und geometrisch bedingte Verzerrungen (Hümmeke et al. 2003). Anhand von Messschablonen, wie z.B. beim Kugelmessröntgen, kann dieser Verzerrungsmaßstab berechnet werden (Solar und Gahleitner 1999). Größenangaben von Metallkugeln sind dabei präzise möglich. In dieser Studie wurden die gemessenen Werte mit einem Vergrößerungsfaktor von 1:1,3 anschließend berechnet. Es ist naheliegend, dass ungenaue Berechnungen des Verzerrungsmaßstabes bei fehlenden Messschablonen zu diesem statistisch signifikanten Unterschied zwischen der OPG und den anderen drei Verfahren geführt haben könnten. Diese Ergebnisse werden von zwei unterschiedlichen Studien bestätigt, in dem gezeigt werden konnte, dass die Zuverlässigkeit der OPG aufgrund der geometrisch bedingten Verzerrungen und der vergrößerten abgebildeten Darstellung limitiert ist (ten Bruggenkate et al.1989, Batenburg et al.1997). In einer weiteren Studie wurden unter anderem die CT mit der OPG anhand der Darstellbarkeit der Lokalisation des Mandibularkanals im Rahmen präimplantologischer Diagnostik verglichen. Dabei konnte deutlich gemacht werden, dass die Messungen anhand der CT der OPG überlegen ist (Peker et al. 2008). Für klinisch einfache Fälle hingegen, in denen der vorliegende Kieferknochen ausreichende Substanzbreite und -höhe hat, sind sowohl die OPG wie auch intraorale Aufnahmeverfahren als diagnostisches Mittel ausreichend (Reddy et al. 1994, BouSerhal et al. 2002).

Für die CBCT-Technologie konnte bereits gezeigt werden, dass es im Vergleich zu Distanzmessungen an menschlichen Schädeln zu statistisch signifikanten Messabweichungen kommen kann, jedoch können die Messwerte der CBCT für die klinische Anwendung als suffizient erachtet werden (Periago et al. 2008). Auch in dieser Studie liegen für den klinischen Gebrauch vernachlässigbar geringe Messabweichungen vor, so dass gute Messwerte erzielt werden können. Ähnliche Ergebnisse konnten in weiteren Studien ermittelt werden, die sich mit der Genauigkeit von linearen (Pinsky et al. 2006, Moshiri et al. 2007, Kumar et al. 2007, Lagravère et al. 2008, Stratemann et al. 2008) volumetrischen (Pinsky et al. 2006) und Winkel-Messungen (Kumar et al. 2007, Lagravère et al. 2008) befasst haben. Anhand linearer Messungen von Knochendefekten konnte gezeigt werden, dass die CBCT ein genaues diagnostisches Verfahren zur Beurteilung von kleinen 
knöchernen Defekten darstellt (Pinsky et al. 2006). Dabei wurden

Messabweichungen zwischen $-0,01 \mathrm{~mm}$ bis $-0,27 \mathrm{~mm}$ für Breite und Höhe ermittelt. Ähnliche Ergebnisse erzielten Stratemann et al. bei dem Vergleich linearer

Messungen mit der CBCT zu physikalischen Messungen. Aufgrund der sehr geringen Messabweichungen von $0,00 \mathrm{~mm}$ bis $0,07 \mathrm{~mm}$ erklärten sie diese für klinisch nicht relevant (Stratemann et al. 2008).

Für die fpVCT gilt, dass Distanzen in allen Ebenen suffizient bestimmt werden können, so dass sich ein entsprechender Sicherheitsabstand zu bei der Implantation gefährdeten Strukturen, wie z.B. der N. alveolaris inferior, hinreichend ermitteln lässt.

Bei der Detektion von Knochen- und Implantatdefekten und den unterschiedlichen Fremdmaterialien lagen keine signifikanten Unterschiede zwischen allen vier Verfahren vor. Auch mit den 2D-Verfahren können diese ausreichend befundet werden. Jedoch sind Erkenntnisse über das Knochenangebot oder Defekte in transversaler Richtung mit ZF und OPG nicht möglich. So waren z.B. bei Präparat 14 keine exakten Aussagen über die Art und räumliche Ausbreitung des Knochendefektes möglich. Auch die Genauigkeit der DVT war nicht ausreichend, um den Defekt differenziert beurteilen zu können. Hingegen war der Defekt (Bonesplitting= künstliche Spaltung des Alveolarkammes bei Implantatinsertion) mit der fpVCT exakt zu beurteilen, da diese Technologie im Vergleich mit der höchsten Ortsauflösung und einer verzerrungsfreien dreidimensionalen Darstellung einhergeht. Insgesamt zeigte sich, dass es bei der zweidimensionalen Darstellung der Objekte zu Überlagerungen von Strukturen kommen kann und damit Defekte sowohl an Alveole als auch am Implantat nicht in allen Fällen suffizient detektiert werden können. Je nach Positionierung der Defekte im Röntgenstrahl war eine Beurteilung möglich oder stark eingeschränkt bis unmöglich. Die 3D-Verfahren waren zwar statistisch gesehen nicht signifikant überlegen, doch oftmals ist die zweidimensionale Darstellung der OPG zur zweifelsfreien Deutung dreidimensionaler Strukturen nicht ausreichend (Garg und Vicari 1995, Ziegler et al. 2002, Patel et al. 2009). Dies trifft vor allem dann zu, wenn das vorliegende Knochenangebot nicht ausreichend vorhanden ist oder speziell dann, wenn eine Ausnutzung der maximal möglichen Implantatlänge therapeutisch gewünscht ist (Reddy et al. 1994). 
Die fpVCT stellt ein ideales Verfahren zur Evaluation von Knochendefekten dar. Auch durch die DVT lassen sich Defekte gut beurteilen, was mit den Ergebnissen von Vandenberghe et al. übereinstimmt (Vandenberghe et al. 2008).

Bei einer Dichte der Dentalimplantate von etwa 3000 HE konnten zudem strukturelle Veränderungen von im Strahlengang vor und hinter dem Implantat liegenden Objekten mit ZF und OPG nicht ausreichend beurteilt werden. Implantatdefekte waren in einer solchen Situation der Diagnostik nicht zugänglich. So waren Querrillen nur evaluierbar, wenn diese genau nach mesial oder distal im Strahlengang ausgerichtet waren. Standarddarstellungsverfahren wie OPG und ZF führen immer zu einer überlagernden Darstellung des abzubildenden Objektes, da sämtliche im Strahlengang liegenden Gewebe und Strukturen zu einer ihrer Elektronendichte entsprechenden Absorption der Röntgenstrahlung führen und somit als Summationseffekt abgebildet werden. Können also bei komplexen Fragestellungen bedeutende anatomische Strukturen durch die Röntgenbilder konventioneller Methoden wie OPG oder ZF nicht eindeutig beurteilt werden, wird ein dreidimensionales bildgebendes Verfahren zur exakten Darstellung der anatomischen Strukturen notwendig (Garg und Vicari 1995, Heurich et al. 2002, Ziegler et al. 2002, Schulze et al. 2005, Voßhans 2005, Monsour und Dudhia 2008, Patel et al. 2009). Hier liegt ein deutlicher Vorteil der fpVCT-Methode, da durch die Einsicht von 3 Dimensionen und der hohen Ortsauflösung auch kleinste Hochkontraststrukturen einsehbar sind. Die DVT ermöglicht zwar ebenso die 3DDarstellung, jedoch sind aufgrund der geringeren Ortsauflösung Strukturen wie planierte Gewindegänge oder Längsrillen eines Implantates nur begrenzt evaluierbar. Überlagernde Darstellungen, die in den zweidimensionalen Verfahren (OPG, ZF) negativen Einfluss auf die Bildauswertung hatten, können durch Untersuchungen mit der fpVCT vermieden werden. Dies ist vergleichbar mit den Aussagen von Kiessling et al. in ihrer Studie zur Überwachung der Tumorangiogenese (Kiessling et al. 2004). Weitere Studien zur qualitativen Bewertung der fpVCT in Bezug auf Überlagerungen von Weich- und Hartgewebsstrukturen liegen jedoch noch nicht vor, sind aber erstrebenswert.

Hingegen liegen einige Studiendaten über die überlagerungsfreie Darstellung von Zahngewebsstrukturen durch DVT vor. So konnten Mengel et al. bereits anhand von periimplantären und peridontalen Defekten zeigen, dass durch die dreidimensionale Bildgebung mit Hilfe von DVT oder CT alle 3 räumlichen Ebenen sehr präzise ohne 
Überlagerungen und Verzerrungen darstellbar sind (Mengel et al. 2005, Mengel et al. 2006).

Bei der Detektion des Fremdmateriales konnte mit allen Methoden in den meisten Fällen eine Aussage über vorhandenes Material und deren Lokalisation getroffen werden. Jedoch war eine genaue Differenzierung nicht immer eindeutig möglich. So führte eine annähernd gleiche Elektronendichte der Guttapercha auch zu Verwechslungen mit den Metallkugeln. Lediglich die Form ließ in vielen Fällen Rückschlüsse auf die Art der Materialen zu. Während es den Untersuchern fast vollständig gelang, Metallkugeln klar zu definieren und die Durchmesser korrekt anzugeben, war das Guttapercha in weniger als der Hälfte der Fälle detektierbar. Das Vorhandensein von B-TCP war aufgrund seiner Körnung und Dichte nur sehr schwer vom Knochen differenzierbar. Ursächlich hierfür ist die zur MSCT vergleichsweise geringe DQE der fpVCT und die daraus resultierende niedrigere Niedrigkontrastauflösung.

Zusammenfassend konnte festgestellt werden, dass mit Hilfe einer dritten Dimension genauere Aussagen hinsichtlich des Vorhandenseins der Lokalisation und der Art der Fremdmaterialien getroffen werden können, als es die 2D-Verfahren ermöglichen. In Abhängigkeit von der Röntgendichte lassen sich die Fremdmaterialien mit den verschiedenen Verfahren unterschiedlich gut detektieren. Im Vergleich von CBCT und herkömmlichen Computertomographen konnte gezeigt werden, dass die DVT ebenso gut hoch radiopake Fremdkörper darstellen kann. Hingegen lassen sich Fremdmaterialien mit einer geringen Elektronendichte mit beiden Verfahren nur ungenügend detektieren (Eggers et al. 2006).

Die heute klinisch einsetzbaren DVT-Geräte ermöglichen ebenso wie die fpVCT eine überlagerungs- und verzerrungsfreie Bilddarstellung (Mengel et al. 2005, Mah und Hatcher 2004). Die DVT der verschiedenen Hersteller kennzeichnen sich dabei durch unterschiedliche Volumina, Auflösungsvermögen, verschiedene Scanzeiten und Strahlendosisbelastungen.

Sowohl für die Implantatplanung als auch für endodontische Fragestellungen eignet sich der NewTom QR-DVT 9000 sehr gut (Stavropoulos und Wenzel 2007, Bernardes et al. 2009). Hochkontraststrukturen lassen sich im Rahmen präoperativer Implantationsdiagnostik und -planung präzise darstellen (Dreiseidler et al. 2009). Anhand der post-mortem Untersuchung fünf humaner Köpfe konnte gezeigt werden, dass die Bildqualität eines CBCTs jüngerer Generation (3D Accuitomo) die eines 
Spiral-CT und die des NewTom QR-DVT 9000 (das erste kommerzielle CBCT) überschreitet (Hirsch et al. 2003). Auch wurde mehrfach die durch CBCT generierte ausgezeichnete Bilddatenerfassung verschiedener Strukturen wie die Morphologie des Unterkiefers, die Lokalisation des Canalis mandibulae und das Verhältnis von röntgendichten Schablonen zum Knochen beschrieben (Peker et al. 2008, Hashimoto et al. 2003, Ito et al. 2001, Nakagawa et al. 2002). Ebenso lassen sich periapikale pathologische Prozesse mit dem 3D Accuitomo gut evaluieren (LofthagHansen et al. 2007).

Ein weiterer relevanter Aspekt bei der Beurteilung von Röntgenaufnahmen ist das Vorliegen von Artefakten, die zu Beeinträchtigungen der Bildauswertung führen können. Metallisches Material hat aufgrund seiner hohen Dichte die Eigenschaft Aufhärtungsartefakte auszulösen, was durch Änderungen des

Röntgenstrahlenspektrums beim Durchdringen des Objektes bedingt wird.

Umliegende Strukturen werden dadurch überstrahlt dargestellt.

Sowohl ZF als auch OPG sind hiervon unwesentlich betroffen. Da bei computertomographischen Verfahren bedingt durch die hohe Absorption eines Titanimplantates im weiteren Strahlengang Aufhärtungsartefakte entstehen, ist die Evaluation der unmittelbar periimplantären Region sowie der Region zwischen Implantaten in Strahlengangsrichtung nur sehr eingeschränkt möglich (Zhang et al. 2007, DGZMK 2009). Bei den DVT-Aufnahmen zeigten sich auch hier Aufhärtungsartefakte durch die Implantate, die in etwa 2/3 zu einer Beeinträchtigung der Bildauswertung geführt haben, was den Aussagen von Draenert et al. und Stavropoulos et al. ähnelt (Draenert et al. 2007, Stavropoulos und Wenzel 2007). Holberg et al. hingegen postulierten, dass Metallartefakte im DVT im Vergleich zum Dental-CT nur sehr abgeschwächt auftreten, während jedoch Störungen der Bildqualität durch Bewegungsartefakte ausschließlich bei der DVT zu sehen waren (Holberg et al. 2005). Bei den Auswertungen der fpVCT wurden hingegen in etwa der Hälfte Einschränkungen der Bildqualität aufgrund von Artefakten durch die Implantate beschrieben.

Immer häufiger werden Radiologen wieder in der bildgebenden Diagnostik der Zahn-, Mund- und Kieferheilkunde eingesetzt, was unter anderem an der zunehmenden Verbreitung von enossalen Implantaten liegt. Dies war ausschlaggebend dafür, bei 
der Evaluation der Daten neben zwei zahnärztlichen Chirurgen und Implantologen einen fachärztlichen Radiologen auszuwählen.

Statistisch signifikante Unterschiede zwischen den Ergebnissen, die durch die einzelnen Beobachter erhoben wurden, lagen nicht vor. Kritisch zu bemerken ist dabei, dass der Radiologe bisher keine Erfahrungen im Umgang mit der Befundung von zahnmedizinischen Röntgenaufnahmen hatte. Zu bedenken ist weiterhin die fehlende Erfahrung aller Beobachter mit der fpVCT-Technologie.

Insgesamt gesehen kommt es zu einer Variabilität der Messergebnisse, da mehrere Personen an der Beurteilung der Daten beteiligt waren. Es konnte bereits gezeigt werden, dass Distanzmessungen am Bildschirm großen Abweichungsmöglichkeiten unterliegen, wenn mehrere Untersucher an der Befundung der Bilder beteiligt sind (Lindh et al. 1995, Jacobs et al.1999). Hingegen ist davon auszugehen, dass höhere Untersucherzahlen eine genauere statistische Untersuchung des subjektiven Einflusses ermöglichen.

Heute werden allein in Deutschland mittlerweile mehr als eine Million Implantate pro Jahr mit steigender Tendenz inseriert, wodurch sich neben des Ersatzes einzelner Zähne auch größere Zahnlücken überbrücken lassen und Behandlungen völlig zahnloser Ober- und Unterkiefer möglich sind (DG). Verschiedene Röntgenverfahren tragen dazu bei, eine möglichst exakte, prothetisch orientierte Positionierung der Implantate unter bestmöglicher Ausnutzung des Knochenangebotes zu planen (DGZMK) und unterstützen damit den Implantationserfolg. Die Qualität der für die Implantologie notwendigen Messungen wird umso besser, je höher die tatsächlich erreichte Orts- und Kontrastauflösung und das Signal-Rausch-Verhältnis des Röntgenverfahrens ist.

Derzeit ist die fpVCT aufgrund der noch zu hohen Strahlenbelastung lediglich für den experimentellen Einsatz geeignet. Bedeutend für eine drastische Minderung der Strahldosis wäre die DQE signifikant zu steigern oder neue Algorithmen zu entwickeln, um mit weniger Projektionsdatenbildern und damit weniger Strahlenbelastung auszukommen. Bei Erreichen einer reduzierten Strahldosis ist eine klinische Anwendung vorstellbar, wobei die fpVCT-Technologie damit eine sehr innovative Aufnahmetechnik für die Klinik darstellt. Durch die hervorragende dreidimensionale, zerstörungsfreie Visualisierung enossaler Implantate und periimplantärer Strukturen könnte sich eine Optimierung des diagnostischen und 
therapeutischen Vorgehens im Rahmen der Implantationsdiagnostik und Behandlungsplanung ergeben.

DVT erfüllen derzeit den Anspruch der dreidimensionalen verzerrungs- und überlagerungsfreien Darstellung von Zahngewebsstrukturen und eignen sich damit ebenfalls für die Diagnostik der Implantationsplanung. Sie erreichen jedoch nicht die hohe Ortsauflösung der fpVCT. Die konventionellen zweidimensionalen Verfahren wie intraoraler ZF und OPG lassen hingegen aufgrund ihrer Zweidimensionalität nur eine limitierte Darstellung der Objekte zu. Sie ermöglichen jedoch eine differenzierte Beurteilung kleiner Strukturen, so dass sie in klinisch einfachen Fällen diagnostisch ausreichend sind und hinsichtlich ihrer Strahleneffektivität und ihrer stärkeren Verbreitung den dreidimensionalen Verfahren überlegen sind.

- Konventionelle 2D-Verfahren wie intraoraler ZF und OPG ermöglichen eine differenzierte Darstellung auch kleiner Strukturen, jedoch sind sie in ihrer Darstellung durch die fehlende 3. Dimension eingeschränkt einsetzbar. Bei ausreichender Substanzbreite und -höhe des Kieferknochens sind sie als diagnostisches Mittel im Rahmen der Implantologie durchaus ausreichend.

- 3D-Verfahren stellen aufgrund ihrer Darstellung der 3. Dimension das Darstellungsverfahren der Zukunft dar.

- DVT ermöglichen eine überlagerungs- und verzerrungsfreie Darstellung dreidimensionaler Strukturen, erreichen jedoch eine geringere Ortsauflösung als die fpVCT-Technologie.

- Die fpVCT-Technologie ermöglicht eine hochauflösende, zerstörungs- und verzerrungsfreie Darstellung kleinster Objekte und Strukturen.

- Die fpVCT ist derzeit nur präklinisch experimentell einsetzbar.

- Die fpVCT ginge derzeit noch mit einer zu hohen effektiven Strahlendosisbelastung für die klinische Anwendung einher, DVT-Geräte ermöglichen hingegen eine 3D-Darstellung mit adäquater Strahlenbelastung.

- Bei Erreichen einer drastischen Reduktion der Strahlendosis durch die signifikante Steigerung der DQE oder der Entwicklung neuer Algorithmen, um mit weniger Projektionsdatenbildern auszukommen, ist eine klinische Anwendung der fpVCT in der Zahn-, Mund- und Kieferheilkunde denkbar. 


\section{Zusammenfassung}

Fragestellung: Die Grundlage einer erfolgreichen Implantatinsertion ist neben dem klinischen Befund die adäquate radiologische Darstellung des geplanten Insertionsortes. Ziel dieser Studie war die Evaluation der Darstellbarkeit von enossalen Implantaten und benachbarten Strukturen mit der FlächendetektorVolumencomputertomographie (fpVCT) im Vergleich zu derzeit klinisch anwendbaren radiologischen Verfahren in der Zahnmedizin.

Methodik: Es wurden insgesamt 14 in Schweinekieferproben inserierte enossale Implantate und die umgebenden Strukturen mit einem Prototypen eines fpVCT untersucht. Die erhobenen Befunde wurden mit intraoralem Zahnfilm (ZF), Orthopantomographie (OPG) und Digitaler Volumentomographie (DVT) verglichen. Die Evaluation der Daten erfolgte anschließend randomisiert geblindet durch zwei zahnärztliche Chirurgen/ Implantologen und einen Radiologen.

Ergebnisse: Bei der Erhebung der Daten waren die 3D-Verfahren den 2D-Verfahren aufgrund der überlagerungsfreien Darstellung deutlich überlegen. Die Implantate und periimplantären anatomischen Strukturen, nebst Knochen- und Implantatdefekten und Fremdmaterial, ließen sich mit der fpVCT adäquat detektieren. Die Knochenund Implantatdefekte konnten hinsichtlich ihrer genauen Lokalisation, Größendimension und Beziehung zu umliegenden Strukturen sehr detailliert beschrieben werden. Differenzierte Aussagen über Art, Anzahl und Größe verschiedener Fremdmaterialien waren möglich. Distanzmessungen ließen sich präzise ausführen. Die genaue Beurteilung von Zahnschmelz, Dentin, Pulpa und dem Parodontalspalt war möglich.

Gegenüber in der Klinik etablierten DVT kann bei Verwendung von Flachbilddetekoren zur computertomographischen Bildgebung eine deutlich höhere räumliche Auflösung bis zu $200 \mu \mathrm{m}$ (bei 10\% MTF) erreicht werden. Der Zahnfilm hingegen ermöglicht durch seine hohe Ortsauflösung ebenso eine präzise Darstellung im Mikrometerbereich, stellt nachteilig jedoch wie ein OPG zweidimensionale Summationsdatenbilder dar. Sowohl Zahnfilm als auch OPG gehen vorteilig mit einer deutlich geringeren Strahlenbelastung für den Patienten bei deutlich höherer Verfügbarkeit einher. Im Vergleich von DVT und fpVCT kommt es bei der fpVCT zu geringeren Einschränkungen der Bildauswertung durch 
Streustrahlenartefakte. Allerdings ist derzeit aufgrund fehlender lokaler Strahlenapplikation eine klinische Anwendung noch nicht möglich.

Die Befunde der verschiedenen Untersucher unterschieden sich nicht. Schlussfolgerung: Die vorliegende Studie zeigt, dass die fpVCT die präzise zerstörungsfreie dreidimensionale Darstellung von Zahnimplantaten und periimplantären Strukturen ermöglicht. 


\section{$\underline{\text { 6. Literaturverzeichnis }}$}

Abrahams JJ (1999): Dental-CT bei pathologischen Veränderungen im Bereich des Kiefers. Radiologe 39, 1035-1043

Albrektsson T, Wennerberg A (2004): Oral implant surfaces: Part 1--review focusing on topographic and chemical properties of different surfaces and in vivo responses to them. Int J Prosthodont 17, 536-543

Andreiotelli M, Wenz HJ, Kohal RJ (2009): Are ceramic implants a viable alternative to titanium implants? A systematic literature review. Clin Oral Implants Res $\underline{20}$, 32-47

Arai Y, Tammisalo E, Iwai K, Hashimoto K, Shinoda K (1999): Development of a compact computed tomographic apparatus for dental use.

Dentomaxillofac Radiol 28, 245-248

Arai Y, Honda K, Iwai K, Shinoda K (2001): Practical model „3DX“ of limited conebeam $\mathrm{X}$ - ray CT for dental use. Int Congr Ser $\underline{1230}, 713-718$

Aranyarachkul P, Caruso J, Gantes B, Schulz E, Riggs M, Dus I, Yamada JM, Crigger M (2005): Bone density assessments of dental implant sites: 2. Quantitative cone-beam computerized tomography. Int J Oral Maxillofac Implants $\underline{20}$, 416-424

Auluck A, Pai KM, Mupparapu M (2007): Multiple mandibular nerve canals: radiographic observations and clinical relevance. Report of 6 cases. Quintessence Int $\underline{38}, 781-787$

Bahat O (2000): Branemark system implants in the posterior maxilla: clinical study of 660 implants followed for 5 to 12 years. Int J Oral Maxillofac Implants $\underline{15}, 646$ - 653

Balshi SF, Wolfinger GJ, Balshi TJ (2007): A retrospective analysis of 44 implants with no rotational primary stability used for fixed prosthesis anchorage.

Int J Oral Maxillofac Implants $\underline{22}, 467$ - 471 
Bartling SH, Majdani O, Gupta R, Rodt T, Dullin C, Fitzgerald PF, Becker H (2007): Large scan field, high spatial resolution flat-panel detector based volumetric CT of the whole human skull base and for maxillofacial imaging.

Dentomaxillofac Radiol $\underline{36}$, 317-327

Batenburg RHK, Stellingsma K, Raghoebar GM, Vissink A (1997): Bone height measurements on panoramic radiographs. The effect of shape and position of edentulous mandibles.

Oral Surg Oral Med Oral Pathol Oral Radiol Endod 4ㅗ, 430-435

Beer A, Gahleitner A, Holm A, Tschabitscher M, Homolka P (2003): Correlation of insertion torques with bone mineral density from dental quantitative $C T$ in the mandible. Clin Oral Implants Res 14, 616-620

Bernardes RA, de Moraes IG, Húngaro Duarte MA, Azevedo BC, de Azevedo JR, Bramante CM (2009): Use of cone-beam volumetric tomography in the diagnosis of root fractures. Oral Surg Oral Med Oral Pathol Oral Radiol Endod 108, 270-277

Bitschnau S, Oberholzer K, Kreitner KF, Otto G, Thelen M, Mildenberger P (2004): CT-Angiographie an einem 16-Schicht-CT zur perioperativen Evaluation der Leberarterien im Vergleich zur MR-Angiographie.

RöFo 176, 1634-1640

Böhme E, Steinbigler P, Czernik A, Scherzberg- Doktorczyk A, Buck J, Haberl R (2003): Invasive versus nichtinvasive (MSCT) Koronarangiographie.

Herz $\underline{28}, 36-43$

BouSerhal C, Jacobs R, Quirynen M, van Steenberghe D (2002): Imaging technique selection for the preoperative planning of oral implants. A review of the literature. Clin Implant Dent Relat Res $\underline{4}, 156-172$

Branemark PI, Hansson BO, Adell R, Breine U, Lindstrom J, Hallen O, Ohman A (1977): Osseointegrated implants in the treatment of the edentulous jaw. Experience from a 10-year period. Scand J Plast Reconstr Surg 16, 1-132 
Brix G, Nagel HD, Stamm G, Veit R, Lechel U, Griebel J, Galanski M (2003):

Radiation exposure in multi-slice versus single-slice spiral $\mathrm{CT}$ : results of a nationwide survey. Eur Radiol 13, 1979-1991

Brooks SL (2005): Effektive dose of two cone-beam CT scanners: I-cat and NewTom 3G. Am Assoc Dent Maxillofac Radiographic Technicians Newsletter, www. aadmrt.com

Brunski JB (1992): Biomechanical factors affecting the bone-dental implant interface. Clin Mater 10, 153-201

Bschorer R, Fuhrmann A, Gehrke G, Keese E, Uffelmann U (1993): Die Darstellung des Canalis mandibulae mit der Unterkieferquerschnitt-Panoramatechnik.

Dtsch Zahnärztl Z $\underline{48}, 786-798$

Buser D, Weber HP, Brägger U (1990): The treatment of partially edentolous patients with ITI hollow-screw implants: presurgical evaluation and surgical procedures. Int J Oral Maxillofac Implants $\underline{5}, 165-174$

Butterfield KJ, Dagenais M, Clokie M (1997): Linear tomographys clinical accuracy and validity for presurgical dental implant analysis.

Oral Surg Oral Med Oral Pathol Oral Radiol Endodont 84, 203-209

Chau AC, Fung K (2009): Comparison of radiation dose for implant imaging using conventional spiral tomography, computed tomography, and cone-beam computed tomography. Oral Surg Oral Med Oral Pathol Oral Radiol Endod 107, 559-565

Chung WE, Rubenstein JE, Phillips KM, Raigrodski AJ (2009): Outcomes assessment of patients treated with osseointegrated dental implants at the University of Washington Graduate Prosthodontic Program, 1988 to 2000.

Int J Oral Maxillofac Implants 노, 927-935 
Cohenca N, Simon JH, Mathur A, Malfaz JM (2007 a): Clinical indications for digital imaging in dento-aveolar trauma. Part 2: root resorption.

Dent Traumatol $\underline{23}, 105-113$

Cohenca N, Simon JH, Roges R, Morag Y, Malfaz JM (2007 b): Clinical indications for digital imaging in dento-alveolar trauma. Part 1: traumatic injuries.

Dent Traumatol $\underline{23}, 95-104$

Cooper LF (2010): Factors influencing primary dental implant stability remain unclear. J Evid Based Dent Pract $\underline{10}$, 44-45

De Backer Al, Mortelé KJ, De Keulenaer BL (2004): Picture archiving and communication system--Part one: Filmless radiology and distance radiology. JBR-BTR $\underline{87}$, 234-241

Degidi M, Piattelli A, Shibli JA, Perrotti V, lezzi G (2009): Early bone formation around immediatly restored implants wit and without occlusal contact: a human histologic and histomorphometric evaluation. Case report.

Int J Oral Maxillofac Implants 24, 734 - 739

DGI - Deutsche Gesellschaft für Implantologie im Zahn-, Mund- und Kieferbereich e.V., DGI München 2009

DGZMK - Deutsche Gesellschaft für Zahn-, Mund- und Kieferheilkunde, Leitlinien, S1-Empfehlung: Dentale Volumentomographie (DVT), DGZMK Düsseldorf 2009

Dixon RL (2003): A new look at CT dose measurement: beyond CTDI. Med Phys $\underline{30}, 1272-1280$

Draenert F, Coppenrath E, Herzog P, Müller S, Mueller-Lisse UG (2007): Beam hardening artefacts occur in dental implant scans with the NewTom cone beam CT but not with the dental 4-row multidetector CT.

Dentomaxillofac Radiol 36, 198-203 
Dreiseidler T, Mischkowski RA, Neugebauer J, Ritter L, Zöller JE (2009): Comparison of cone-beam imaging with orthopantomography and computerized tomography for assessment in presurgical implant dentistry.

Int J Oral Maxillofac Implants 로, 216-225

Eggers G, Welzel T, Mukhamadiev D, Wörtche R, Hassfeld S, Mühling J (2006): Xray-based volumetric imaging of foreign bodies: a comparison of computed tomography and digital volume tomography. J Oral Maxillofac Surg $\underline{65}, 1880-1885$

Ekestubbe A, Grondahl K, Ekholm S, Johansson PE, Grondahl HG (1996):

Low-dose tomographic techniques for dental implant planning.

Int J Oral Maxillofac Implants 11, 650-659

Ekestubbe A, Grondahl K, Grondahl HG (1997): The use of tomography for dental implant planning. Dentomaxillofac Radiol 26, 206-213

Engelke K, Karolczak M, Lutz A, Seibert U, Schaller S, Kalender W (1999): Mikro-CT technology and application for assessing bone structure. Radiologie 39, 203-212

Ericsson I, Nilner K (2002): Early functional loading using Branemark dental implants. Int J Periodontics Restorative Dent 22, 9-19

Esposito M, Grusovin MG, Martinis E, Coulthard P, Worthington HV (2007): Interventions for replacing missing teeth: 1- versus 2-stage implant placement. Cochrane Database Syst Rev 18, CD006698

Farman AG, Scarfe WC (2006): Development of imaging selection criteria and procedures should precede cepahlometric assessment with cone beam computed tomography. Am J Orthod Dentofacial Orthop 130, 257-265

Flinzberg S, Schmelzle R, Schulze D, Rother U, Heiland M (2003): 3-dimensional imaging possibilities of the mid-face area using digital volume tomography based on a daver study of angle stable osteosynthesis. Mund Kiefer Gesichtschir $\underline{7}, 289-293$ 
Flohr T, Stierstorfer K, Raupach R, Ulzheimer S, Bruder H (2004): Performance Evaluation of a 64-Slice CT System with z-Flying Focal spot.

RöFo 176, 1803-1810

Flohr T, Ohnesorge B, Stierstorfer K, Bruder H, Simon J, Süß C, Wildberger J, Baum U, Lell M, Küttner A, Heuschmid M, Wintersperger B, Becker C, Schaller S (2005 a): Auf dem Weg zur isotropen räumlichen Auflösung: Technische Grundlagen und Anwendungen der 16-Schicht-CT. Radiologe $\underline{45}$, 608-617

Flohr TG, Schaller S, Stierstorfer K, Bruder H, Ohnesorge BM, Schoepf UJ (2005 b): Multi-detector row CT systems and image-reconstruction techniques. Radiology $\underline{235}$, 756-773

Frederiksen NL (1995): Diagnostic imaging in dental implantology. Oral Surg Oral Med Oral Pathol Oral Radiol Endod 80, 540-554

Fu KY, Zhang WL, Liu DG, Chen HM, Ma XC (2007): Cone beam computed tomography in the diagnosis of temporomandibular joint osteoarthrosis. Zhonghua Kou Qiang Yi Xue Za Zhi; 42, 417-420

Fuh LJ, Huang HL, Chen CS, Fu KL, Shen YW, Tu MG, Shen WC, Hsu JT (2010): Variations in bone density at dental implant sites in different regions of the jawbone. J Oral Rehabil, 37, 346-351

Fuhrmann AW, Schulze D, Rother U, Vesper M (2003): Digital transversal slice imaging in dental-maxillo-facial radiology: from pantomography to digital volume tomography. Int J Comput Dent $\underline{6}, 129-140$

Funke M, Hermann KP, Dullin C, Heidrich G, Rieker J, Grabbe E (2005): Hochauflösende Kegelstrahl-Volumen-CT mit zwei Flachbilddetektoren: Methodik und experimentelle Untersuchungen zur Bildqualität. RöFo $\underline{177}, 6-8$

Gahleitner A, Watzek G, Imhof H (2003): Dental CT: imaging technique, anatomy, and pathologic conditions of the jaw. Eur Radiol $\underline{13}, 366-376$ 
Ganz SD (2008): Computer-aided Design/ Computer-aided Manufacturing Applications Using CT and Cone Beam CT Scanning technology. Dent Clin North Am $\underline{52}, 777-808$

Garg AK, Vicari A (1995): Radiographic modalities for diagnosis and treatment planning in implant dentistry. Implant Soc $\underline{5}, 7-11$

Gavalla S, Donta C, Tsiklakis K, Boziari A, Kamenopoulou V, Stamatakis HC (2009): Radiation dose reduction in direct digital panoramic radiography. Eur J Radiol 1ㅡ, 42-48

Gher ME, Richardson AC (1995): The accuracy of dental radiographic techniques used for evaluation of implant fixture placement. Int J Periodontics Restorative Dent $\underline{15}$, 268-283

Gijbels F, Jacobs R, Bogaerts R, Debaveye D, Verlinden S, Sanderink G (2005): Dosimetry of digital panoramic imaging. Part I: Patient exposure.

Dentomaxillofac Radiol 34,145-149

Glauser R, Sennerby L, Meredith N, Ree A, Lundgren A, Gottlow J, Hammerle CH (2004): Resonance frequency analysis of implants subjected to immediate or early functional occlusal loading. Successful vs. failing implants.

Clin Oral Implants Res $\underline{15}$, 428-434

Gómez-Román G, Axmann D, d'Hoedt B, Schulte W (1995): Eine Methode zur quantitativen Erfassung und statistischen Auswertung des periimplantären Knochenabbaus. Stomatologie $\underline{92}, 463$

Greenstein G, Tarnow D (2006): The mental foramen and nerve: clinical and anatomical factors related to dental implant placement: a literature review. J Periodontol 구, 1933-1943 
Greschus S, Kiessling F, Savai R, Ruppert C, Traupe H (2005): Bildgebung mit einem Flächendetektor- Computertomographen in der präklinischen Anwendung. RöFo 177, 460, Abstract S 4.11

Guerrero ME, Jacobs R, Loubele M, Schutyser F, Suetens P, van Steenberghe D (2006): State-of-the-art on cone beam CT imaging for preoperative planning of implant placement. Clin Oral Invest $\underline{10}, 1-7$

Guderjahn T: Visualisierung implantierter Koronarstents im FlächendetektorVolumen-CT am Tiermodell. Med. Diss. Göttingen 2005

Gupta R, Grasruck M, Suess C, Bartling SH, Schmidt B, Stierstorfer K, Popescu S, Brady T, Flohr T (2006): Ultra-high resolution flat-panel volume CT: fundamental principles, design architecture, and system characterization.

Eur Radiol $\underline{16}$, 1191-1205

Gupta R, Cheung AC, Bartling SH, Lisauskas J, Grasruck M, Leidecker C, Schmidt B, Flohr T, Brady TJ (2008): Flat-panel volume CT: fundamental principles, technology, and applications. Radiographics $\underline{28}, 2009-2022$

Hahn W, Fricke-Zech S, Fialka-Fricke J, Dullin C, Zapf A, Gruber R, SennhennKirchner S, Kubein-Meesenburg D, Sadat-Khonsari R (2009 a): Imaging of the midpalatal suture in a porcine model: flat-panel volume computed tomography compared with multislice computed tomography.

Oral Surg Oral Med Oral Pathol Oral Radiol Endod 108, 443-449

Hahn W, Fricke-Zech S, Fricke J, Gruber RM, Dullin C, Zapf A, Hannig C, KubeinMeesenburg D, Sadat-Khonsari R (2009 b): Detection and size differentiation of simulated tooth root defects using flat-panel volume computerized tomography (fpVCT). Oral Surg Oral Med Oral Pathol Oral Radiol Endod 107, 272-278

Hannig C, Dullin C, Hülsmann M, Heidrich G (2005): Three-dimensional, nondestructive visualization of vertical root fractures using flat panel volume detector computer tomography: an ex vivo in vitro case report. Int Endod J $\underline{38}, 904-913$ 
Hannig C, Krieger E, Dullin C, Merten HA, Attin T, Grabbe E, Heidrich G (2006):

Volumetry of human molars with flat-panel based volume CT in vitro.

Clin Oral Investig $\underline{10}, 253-257$

Hashimoto K, Arai Y, Iwai K, Araki M, Kawashima S, Terakado M (2003): A

comparison of a new limited cone beam computed tomography machine for dental use with a multidetector row helical CT machine.

Oral Surg Oral Med Oral Pathol Oral Radiol Endod $\underline{95}, 371-377$

Hashimoto K, Kawashima S, Araki M, Iwai K, Sawada K, Akiyama Y (2006):

Comparison of image performance between cone-beam computed tomography for dental use and four-row multidetector helical CT. J Oral Sci $\underline{48}, 27-34$

Hashimoto K, Kawashima S, Kameoka S, Akiyama Y, Honjoya T, Ejima K, Sawada K (2007): Comparison of image validity between cone beam computed tomography for dental use and multidetector row helical computed tomography.

Dentomaxillofac Radiol $\underline{36}$, 465-471

Heidrich G, Hassepass F, Dullin C, Attin T, Grabbe E, Hannig C (2005 a): Nondestructive, preclinical evaluation of root canal anatomy of human teeth with flatpanel detector volume CT (FD-VCT). RöFo 177, 1683-1690

Heidrich G, Funke M, Dullin C, Hermann KP, Obenauer S, Grabbe E (2005 b): Darstellung von ballonexpandierbaren Stents mit einem Kegelstrahl-Volumen-CTScanner im Vergleich zu einem 8-Zeilen-Spiral-CT.

RöFo 177, 466, Abstract S 6.7

Hermann KP, Dullin C, Funke M, Grabbe E (2005): Experimentelle Ermittlung der Leistungsmerkmale eines Flächendetektor-Volumen-CT (FD-VCT) für die Kleintierbildgebung. RöFo 177, VO 2090 
Heurich T, Ziegler C, Steveling H, Wortche R, Muhling J, Hassfeld S (2002):

Digital volume tomography - an extension to the diagnostic procedures available for application before surgical removal of third molars.

Mund Kiefer Gesichtschir $\underline{6}$, 427-432

Hirsch E, Graf HL, Hemprich A (2003): Comparative investigation of image quality of three different $\mathrm{X}$-ray procedures; Dentomaxillofac Radiol $\underline{32}$, 201-211

Hirsch E, Wolf U, Heinicke F, Silva MA (2008): Dosimetry of the cone beam computed tomography Veraviewepocs 3D compared with the 3D Accuitomo in different fields of view. Dentomaxillofac Radiol 37, 268-273

Hirschfelder U (1994): Radiological survey imaging of the dentition: dental CT versus orthopantomography. J Orofac Orthop $\underline{55}, 14-20$

Holberg C, Steinhäuser S, Geis P, Rudzki-Janson I (2005): Cone-beam computed tomography in orthodontics: benefits and limitations. J Orofac Orthop $\underline{66}$, 434-444

Hollender L: Radiographic examination of endosseous implants in the jaws. In: Worthington P, Branemark P-I, eds. Advanced osseointegration surgery: applications in the maxillofacial region. Quintessence Publishing, Chicago 1992, 80-93

Honda K, Arai Y, Iwai K, Hashimoto K, Saitou T, Shinoda K (2001): Fundamental efficiency of new-style limited-cone-beam CT (3DX) - comparison with helical CT. Jpn J Tomogr 르, 193-198

Honey OB, Scarfe WC, Hilgers MJ, Klueber K, Silveira AM, Haskell BS, Farman AG (2007): Accuracy of cone-beam computed tomography imaging of the temporomadibular joint: comparisons with panoramic radiology and linear tomography. Am J Orthod Dentofacial Orthop 132, 429-438

Hümmeke S, Esser E, Vosshans J (2003): Diagnostische Möglichkeiten der digitalen Volumentomographie. Quintessenz $\underline{64}$, 1105-1112 
Hussain AM, Packota G, Major PW, Flores-Mir C (2008): Role of different imaging modalities in assessment of temporomandibular joint erosions and osteophytes: a systematic review. Dentomaxillofac Radiol $\underline{37}$, 63-71

Imhof H, Dirisamer A, Fischer H, Grampp S, Heiner L, Kaderk M, Krestan C, Kainberger F (2002): Prozessmanagementänderung durch den Einsatz von RIS, PACS und Festkörperdetektoren. Radiologe $\underline{42}, 344-350$

Iplikcioglu H, Akca K, Cehreli MC (2002): The use of computerized tomography for diagnosis and treatment planning in implant dentistry. J Oral Implantol $\underline{28}, 29-36$

Ito K, Gomi Y, Sato S, Arai Y, Shinoda K (2001): Clinical application of a new compact CT system to assess 3-D images for the preoperative treatment planning of implants in the posterior mandible. A case report. Clin Oral Implants Res $\underline{12}, 539-542$

Jacobs R, van Steenberghe D: Radiographic planning and assessment of endosseous oral implants. Springer-Verlag, Berlin 1998

Jacobs R, Adriansens A, Verstreken K, Suetens P, van Steenberghe D (1999): Predictability of a three-dimensional planning system for oral implant surgery. Dentomaxillofac Radiol $\underline{28}, 105-111$

Jivraj S, Chee W (2006): Rationale for dental implants. Br Dent J 200, 661-665

Jokstad A, Braegger U, Brunski JB, Carr AB, Naert I, Wennerberg A (2003): Quality of dental implants. Int Dent J $\underline{53}, 409-443$

Kaeppler G, Axman-Krcmar D, Reuter I, Meyle J, Gomez-Roman G (2000): A clinical evaluation of some factors affecting image quality in panoramic radiography. Dentomaxillofac Radiol $\underline{29}$, 81-84

Kalender WA (2003): Der Einsatz von Flachbilddetektoren für die CT- Bildgebung. Radiologie $\underline{43}, 379-387$ 
Kalender WA, Kyriakou Y (2007): Flat-detector computed tomography (FD-CT).

Eur Radiol 17, 2767-2779

Kalender WA, Polacin A, Süss C (1994): A comparison of conventional and spiral CT: an experimental study on the detection of spherical lesions.

J Comput Assist Tomogr 18, 167-176

Kamburoğlu K, Ilker Cebeci AR, Gröndahl HG (2009): Effectiveness of limited conebeam computed tomography in the detection of horizontal root fracture.

Dent Traumatol 25, 256-261

Keller U (1995): Die Darstellung des periimplantären Knochenabbaus im Röntgenbild. Z Zahnärztl Implantol 11, 88

Kiessling F, Greschus S, Lichy MP, Bock M, Fink C, Vosseler S, Moll J, Mueller MM, Fusening NE, Traupe H, Semmler W (2004): Volumetric computed tomography (VCT): a new technology for noninvasive, high-resolution monitoring of tumor angiogenesis. Nat Med $\underline{10}, 1133-1138$

King KS, Lam EW, Faulkner MG, Heo G, Major PW (2007): Vertical bone volume in the paramedian palate of adolescents: a computed tomography study.

Am J Orthod Dentofacial Orthop 132, 783-788

Kobayashi K, Shimoda S, Nakagawa Y, Yamamoto A (2004): Accuracy in measurements of distance using limited cone- beam computerized tomography. Int J Oral Maxillofac Implants $\underline{19}$, 228-231

Koeck B, Wagner W: Diagnostik, Planung, Aufklärung; in: Implantologie, 2. Auflage; hrsg. v. Koeck B, Wagner W unter Mitarbeit namhafter Autoren, Urban \& Fischer Verlag, München 2004, 29-46

Kotter E, Langer M (2002): Digital radiography with large-area flat-panel detectors. Eur Radiol $\underline{12}$, 2562-2570 
Kumar V, Ludlow JB, Mol A, Cevidanes L (2007): Comparison of conventional and cone beam CT synthesized cephalograms.

Dentomaxillofac Radiol 36, 263-269

Kyriakou Y, Deak P, Langner O, Kalender WA (2008): Concepts for dose determination in flat-detector CT. Phys Med Biol $\underline{53}$, 3551-3566

Kyriakou Y, Struffert T, Dörfler A, Kalender WA (2009): Grundlagen der Flachdetektor-CT (FD-CT). Radiologe $\underline{49}$, 811-819

Lagravère MO, Carey J, Toogood RW, Major PW (2008): Three-dimensional accuracy of measurements made with software on cone-beam computed tomography images. Am J Orthod Dentofacial Orthop 134, 112-116

Lambert FE, Weber HP, Susarla SM, Belser UC, Gallucci GO (2009): Descriptive analysis of implant and prosthodontic survival rates with fixed implant-supported rehabilitations in the edentulous maxilla. J Periodontol $\underline{80}, 1220-1230$

Lecomber AR, Downes SL, Mokhtari M, Faulkner K (2000): Optimisation of patient doses in programmable dental panoramic radiography.

Dentomaxillofac Radiol $\underline{29}$, 107-112

Lecomber AR, Yoneyama Y, Lovelock DJ, Hosoi T, Adams AM (2001): Comparison of patient dose from imaging protocols for dental implant planning using conventional radiography and computed tomography. Dentomaxillofac Radiol $\underline{30}$, 255-259

Lernkamp M, Filippi A, Berndt D, Lambrecht T (2006): Diagnostische Möglichkeiten der digitalen Volumentomografie. Schweiz Monatsschr Zahnmed 116, 645-650

Lindh C, Petersson A, Klinge B (1995): Measurements of distances related to the mandibular canal in radiographs. Clin Oral Implants Res $\underline{6}, 96-103$

Loewenhardt B, Müller E: Bildgebende Diagnostik, Technik - Anatomie - Pathologie 3. Auflage; Loewenhardt-Verlag, Fulda 2006 
Lofthag-Hansen S, Huumonen S, Gröndahl K, Gröndahl HG (2007): Limited conebeam CT and intraoral radiography for the diagnosis of periapical pathology. Oral Surg Oral Med Oral Pathol Oral Radiol Endod $\underline{103}, 114-119$

Loubele M, Guerrero ME, Jacobs R, Suetens P, van Steenberghe D (2007):

A comparison of jaw dimensional and qualitiy assessments of bone characteristics with cone-beam CT, spiral tomography and multislice computer tomography.

Oral Maxillofac Implants $\underline{22}, 446-454$

Loubele M, Bogaerts R, Van Dijck E, Pauwels R, Vanheusden S, Suetens P Marchal $G$, Sanderink $G$, Jacobs $R$ (2009): Comparison between effective radiation dose of CBCT and MSCT scanners for dentomaxillofacial applications.

Eur J Radiol 1ㅡ, 461-468

Ludlow JB, Ivanovic M (2008): Comparative dosimetry of dental CBCT devices and 64-slice $\mathrm{CT}$ for oral and maxillofacial radiology.

Oral Surg Oral Med Oral Pathol Oral Radiol Endod 106, 930-938

Ludlow JB, Davies-Ludlow LE, Brooks SL, Howerton WB (2006): Dosimetry of 3 CBCT devices for oral and maxillofacial radiology: CB Mercuray, NewTom $3 G$ and iCAT. Dentomaxillofac Radiol 35, 219-226

Ludlow JB, Davies-Ludlow LE, White SC (2008): Patient risk related to common dental radiographic examinations: the impact of 2007 International Commission on Radiological Protection recommendations regarding dose calculation. J Am Dent Assoc 139, 1237-1243

Mah J, Hatcher D (2004): Three-dimensional craniofacial imaging. Am J Orthod Dentofacial Orthop $\underline{126}$, 308-309

Marmulla R, Wörtche R, Mühling J, Hassfeld S (2005): Geometric accuracy of the NewTom 9000 Cone Beam CT. Dentomaxillofac Radiol $\underline{34}$, 28-31 
Marten K, Engelke C, Grabbe E, Rummeny EJ (2004): CT-Bildgebung mit einem Flachbilddetektor: experimentelle Untersuchung zur Wachstumsratenbestimmung von Lungenrundherden. RöFo 176, 752-757

May GA, Deer DD, Dackiewicz D (2000): Impact of digital radiography on clinical workflow. J Digit Imaging 13, 76-78

Mengel R, Candir M, ShiratoriK, Flores-de-Jacoby L (2005): Digital volume tomography in the diagnosis of periodontal defects: an in vitro study on native pig and human mandibles. J Periodontol $\underline{76}, 665-673$

Mengel R, Kruse B, Flores-de-Jacoby L (2006): Digital volume tomography in the diagnosis of peri-implant defects: an in vitro study on native pig mandibles. J Periodontol $\underline{77}, 1234-1241$

Mesa F, Muñoz R, Noguerol B, de Dios Luna J, Galindo P, O'Valle F (2008): Multivariate study of factors influencing primary dental implant stability. Clin Oral Implants Res $\underline{19}, 196-200$

Missbach-Guentner J, Dullin C, Zientkowska M, Domeyer.Missbach M, Kimmina S, Obenauer S, Kauer F, Stühmer W, Grabbe E, Vogel WF, Alves F (2007):

Flat-panel detector-based volume computed tomography: A novel 3D imaging technique to monitor osteolytic bone lesions in a mouse tumor metastasis model. Neoplasia $\underline{9}, 755-765$

Möbes O, Becker J, Schnelle C, Ewen K, Kemper J, Cohnen M (2000):

Strahlenexposition bei der digitalen Volumentomographie,

Panoramaschichtaufnahme und Computertomographie.

Dtsch Zahnärztl Z $\underline{55}$, 336-339

Mol A, Balasundaram A (2008): In vitro cone beam computed tomography imaging of periodontal bone. Dentomaxillofac Radiol 37, 319-324 
Molly L (2006): Bone density and primary stability in implant therapy.

Clin Oral Implants Res 17,124-35

Monsour PA, Dudhia R (2008): Implant radiography and radiology.

Aust Dent J $\underline{53}, 11-25$

Moshiri M, Scarfe WC, Hilgers ML, Scheetz JP, Silveira AM, Farman AG (2007):

Accuracy of linear measurements from imaging plate and lateral cephalometric images derived from cone-beam computed tomography.

Am J Orthod Dentofacial Orthop 132, 550-560

Mozzo P, Procacci C, Tacconi A, Tinazzi Martini P, Bergamo Andreis IA (1998): A new volumetric CT machine for dental imaging based on the cone- beam technique: preliminary results. Eur Radiol $\underline{8}, 1558-1564$

Mraiwa N, Jacobs R, van Steenberghe D, Quirynen M (2003): Clinical assessment and surgical implications of anatomic challenges in the anterior mandible.

Clin Implant Dent Relat Res $\underline{5}, 219$ - 225

Müssig E, Wörtche R, Lux CJ (2005): Indications for digital volume tomography in orthodontics. J Orofac Orthop 66, 241-249

Nakagawa Y, Kobayashi K, Mishima, Ishii H, Asada K, Ishibashi K (2002):

Preoperative application of limited cone beam computerized tomography as an assessment toll before minor oral surgery. Int J Oral Maxillofac Surg 31, 322-327

Nakata K, Naitoh M, Izumi M, Inamoto K, Ariji E, Nakamura H (2006): Effectiveness of dental computed tomography in diagnostic imaging of periradicular lesion of each root of a multirooted tooth: a case report. J Endod $\underline{32}, 583-587$

Nakata K, Naitoh M, Izumi M, Ariji E, Nakamura H (2009): Evaluation of correspondence of dental Computed Tomography imaging to anatomic observation of external root resorption. J Endod $\underline{35}, 1594-1597$ 
Nance R, Tyndall D, Levin LG, Trope M (2000): Identification of root canals in molars by tuned-aperture computed tomography. Int Endod J $\underline{33}, 392-396$

Neff KW, Lehmann KJ, Weisser G, Denk S, Georgi M (1998): Elektronenstrahl-CTAngiographie beim abdominellen Aortenaneurysma. Erste Ergebnisse.

Radiologie $\underline{38}, 1060-1068$

Ning R, Chen B, Yu R, Conover D, Tang X, Ning Y (2000): Flat panel detector-basedcone-beam-volumetric CT angiography imaging: system evaluation.

IEEE Trans Med Imaging 19, 949-963

Ning R, Tang X, Conover D, Yu R (2003): Flat panel detector-based cone beam computed tomography with a circle-plus-two-arcs data acquisition orbit: preliminary phantom study. Med Phys $\underline{30}$, 1694-1705

Obenauer S, Rieker J, Hermann KP, Heidrich G, Funke M, Grabbe E (2005): Vergleich der hochauflösenden Kegelstrahl-Volumen-CT mit der 8-Zeilen-Spiral-CT: Experimentelle Untersuchungen zur Erkennbarkeit von knöchernen Strukturen. RöFo 177, S6_5

Obenauer S, Dullin C, Alves F, Missbach-Guentner J, Grabbe E, Heuser M (2007): Flat-panel-detector-based volumetric CT: performance evaluation of imaging for skeletal structures of small animals in comparison to multislice CT.

Clin Imaging $\underline{31}, 18-22$

Ohnesorge B, Flohr T, Schaller S, Klingenbeck- Regn K, Becker C, Schöpf UJ, RüningG R, Reiser MF (1999): Technische Grundlagen und Anwendung der Mehrschicht CT. Radiologe 39, 923-931

Okano T, Harata Y, Sugihara Y, Sakaino R, Tsuchida R, Iwai K, Seki K, Araki K (2009): Absorbed and effective doses from cone beam volumetric imaging for implant planning. Dentomaxillofac Radiol $\underline{38}$, 79-85 
Oliva J, Oliva X, Oliva JD (2007): One-year follow-up of first consecutive 100 zirconia dental implants in humans: a comparison of 2 different rough surfaces.

Int J Oral Maxillofac Implants ⒉

Oliva J, Oliva X, Oliva JD (2010): Five-year Success Rate of 831 Consecutively Placed Zirconia Dental Implants in Humans: A Comparison of Three Different Rough Surfaces. Int J Oral Maxillofac Implants $\underline{25}$, 336-344

O'Sullivan D, Sennerby L, Meredith N (2004): Influence of implant taper on the primary and secondary stability of osseointegrated titanium implants.

Clin Oral Implants Res $\underline{15}, 474-480$

Patel S (2009): New dimensions in endodontic imaging: Part 2. Cone beam computed tomography. Int Endod J $\underline{42}, 463-475$

Patel S, Dawood A, Ford TP, Whaites E (2007): The potential applications of cone beam computed tomography in the management of endodontic problems. Int Endod J 누, 818-830

Patel S, Dawood A, Whaites E, Pitt Ford T (2009): New dimensions in endodontic imaging: part 1. Conventional and alternative radiographic systems.

Int Endod J 42, 447-462

Peker I, Alkurt MT, Michcioglu T (2008): The use of 3 different imaging methods for the localization of the mandibular canal in dental implant planning.

Int J Oral Maxillofac Implants $\underline{23}, 463-470$

Periago DR, Scarfe WC, Moshiri M, Scheetz JP, Silveira AM, Farman AG (2008):

Linear accuracy and reliability of cone beam CT derived 3-dimensional images constructed using an orthodontic volumetric rendering program.

Angle Orthod $\underline{78}, 387-395$ 
Peters OA, Laib A, Rüegsegger P, Barbakow F (2000): Three-dimensional analysis of root canal geometry by high resolution computed tomography.

J Dent Res $\underline{79}, 1405-1409$

Peters OA, Laib A, Gohring TN, Barbakow F (2001): Changes in root canal geometry after preparation assessed by high resolution computed tomography.

J Endod 27, 1-6

Peters OA, Peters Cl, Schönenberger K, Barbakow F (2003): ProTaper rotary root canal preparation: effects of canal anatomy on final shape analysed by micro CT. Int Endod J 36, 86-92

Pinsky HM, Dyda S, Pinsky RW, Misch KA, Sarment DP (2006): Accuracy of threedimensional measurements using cone-beam CT.

Dentomaxillofac Radiol 35, 410-416

Reddy MS, Mayfield Donahoo T, Vanderven FJ, Jeffcoat MK (1994): A comparison of the diagnostic advantages of panoramic radiography and computed tomography scanning for placement of root form dental implants.

Clin Oral Implants Res $\underline{5}$, 229-238

Rhodes JS, Ford TR, Lynch JA, Liepins PJ, Curtis RV (2000): A comparison of two nickel-titanium instrumentation techniques in teeth using microcomputed tomography. Int Endod J 33, 279-285

Riedel T: Deterministic simulation of arbitrary CT measurements with experimental verification. Shaker Verlag Aachen 2005

Roberts JA, Drage NA, Davies J, Thomas DW (2009): Effective dose from cone beam CT examinations in dentistry. Br J Radiol $\underline{82}, 35-40$

Rodrigo D, Aracil L, Martin C, Sanz M (2010): Diagnosis of implant stability and its impact on implant survival: a prospective case series study.

Clin Oral Implants Res 21, 255-261 
Rogalla P, Kloesters C, Hein PA (2009): CT technology overview: 64-slice and beyond. Radiol Clin North Am $\underline{47}, 1-11$

Ross W, Cody DD, Hazle JD (2006): design and performance characteristics of a digital flat-panel computed tomography system.

Med Phys $\underline{33}$, 1888-1901

Rother U: Moderne bildgebende Diagnostik in der Zahn-, Mund- und Kieferheilkunde, Grundlagen Strahlenschutz Befunde. Urban \& Fischer-Verlag, München 2001

Rothman SLG, Chaftez N, Rhodes ML, Schwarz MS (1988): CT in the preoperative assessment of the mandible and the maxilla for endosseous implant surgery. Radiology $\underline{168}, 171-175$

Rubenstein JE,Taylor TD (1997): Apical nerve transection resulting from implant placement: a 10-year follow-up report. J Prosthet Dent $\underline{78}, 537-541$

Rugani P, Kirnbauer B, Arnetzl GV, Jakse N (2009): Cone beam computerized tomography: basics for digital planning in oral surgery and implantology. Int J Comput Dent 12, 131-145

Scarfe WC, Farman AG, Sukovic P (2006): Clinical applications of cone-beam computed tomography in dental practice. J Can Dent Assoc $\underline{72}, 75-80$

Schaefer-Prokop C, Uffmann M, Sailer J, Kabalan N, Herold C, Prokop M (2003):

Digitale Thoraxradiographie: Flat-panel-Detektor oder Speicherfolie.

Radiologe $\underline{43}, 351-361$

Schima W, Kulinna C, Ba-Ssalamah A, Grünberger T (2005): Multidetektor-CT (MDCT) der Leber. Radiologe 45, 15-23

Schliephake H: Sofortbelastung/ Sofortversorgung. DGI - Deutsche Gesellschaft für Implantologie im Zahn-, Mund- und Kieferbereich e.V., Göttingen-München 2004 
Schmuth GP, Freisfeld M, Köster O,Schüller H (1995). The application of computerized tomography (CT) in cases of impacted maxillary canines. Eur J Orthod 14, 296-301

Schulze D, Heiland M, Schmelzle R, Rother U (2005): Diagnostische Möglichkeiten der digitalen Volumentomographie im Bereich des Gesichtsschädels.

Quintessenz $\underline{56}, 51-56$

Schumacher M, Schulgen G: Methodik klinischer Studien;

Springer-Verlag, Berlin 2002, S. 180

Schwarz MS, Rothman SLGM, Rhodes ML (1987 a): Computed tomography. I. Preoperative assessment of the mandible for endosseous implant surgery.

Int J Oral Maxillofac Implants $\underline{2}, 137-141$

Schwarz MS, Rothman SLGM, Rhodes ML (1987 b): Computed tomography. II. Preoperative assessment of the maxilla for endosseous implant surgery. Int J Oral Maxillofac Implants 2, 143-148

Schwarz MS, Rothman SLG, Chafetz N, Rhodes M (1989): Computed tomography in dental implantation surgery. Dent Clin North Am $\underline{33}, 555-597$

Sennerby L, Meredith N (2008): Implant stability measurements using resonance frequency analysis: biological and biomechanical aspects and clinical implications. Clin Oral Implants Res $\underline{47}, 51-66$

Shope TB, Gagne RM, Johnson GC (1981): A method for describing the doses delivered by transmission $x$-ray computed tomography. Med Phys $\underline{8}$, 488-495

Simon JH, Enciso R, Malfaz J, Roges R, Bailey-Perry M, Patel A (2006): Differential diagnosis of large periapical lesions using cone-beam computed tomography measurements and biopsy. J Endod $\underline{32}, 833-837$ 
Søballe K, Hansen ES, B-Rasmussen H, Jørgensen PH, Bünger C (1992): Tissue ingrowth into titanium and hydroxyapatite-coated implants during stable and unstable mechanical conditions. J Orthop Res $\underline{10}$, 285-299

Solar P, Gahleitner A (1999): Dental- CT zur Planung chirurgischer Eingriffe. Radiologe $\underline{39}, 1051-1063$

Suomalainen A, Kiljunen T, Käser Y, Peltola J, Kortesniemi M (2009): Dosimetry and image quality of four dental cone beam computed tomography scanners compared with multislice computed tomography scanners. Dentomaxillofac Radiol $\underline{38}$, 367-378

Spahn M (2005): Flat detectors and their clinical apploications.

Eur Radiol $\underline{15}$, 1943-1947

Spahn M, Strotzer M, Völk M, Böhm S, Geiger B, Hahm G, Feuerbach S (2000): Digital radiography with a large area, amorpheus silicon flat-panel $\mathrm{X}$-ray detector system. Invest Radiol 35, 260-266

Spahn M, Heer V, Freytag R (2003): Flachbilddetektoren in der Röntgendiagnostik. Radiologe $\underline{43}, 340-350$

Stanford CM (2005): Application of oral implants to the general dental practice. J Am Dent Assoc 136, 1092-1100

Stavropoulos A, Wenzel A (2007): Accuracy of cone beam dental CT, intraoral digital and conventional film radiography for the detection of periapical lesions. An ex vivo study in pig jaws. Clin Oral Investig $\underline{11}, 101-106$

Stratemann SA, Huang JC, Maki K, Miller AJ, Hatcher DC (2008): Comparison of cone beam computed tomography imaging with physical measures.

Dentomaxillofac Radiol 37, 80-93

Tal H, Moses O (1991): A comparison of panoramic radiography with computed tomography in the planning of implant surgery. Dentomaxillofac Radiol $\underline{20}, 40-42$ 
ten Bruggenkate CM, van der Linden LWJ, Oosterbeek HS (1989): Parallelism of implants visualized on the orthopantomogramm. Int J Oral Maxillofac 18, 213-215

Testori T, Galli F, Capelli M, Zuffetti F, Esposito M (2007): Immediate nonocclusal versus early loading of dental implants in partially edentulous patients: 1 -year results from a multicenter, randomized controlled clinical trial.

Int J Oral Maxillofac Implants $\underline{22}, 815-822$

Theisen FC, Shultz RE, Elledge DA (1990): Displacement of a root form implant into the mandibular canal. Oral Surg Oral Med Oral Pathol $\underline{70}, 24-28$

Thiel HJ, Hassfeld S: Schnittbilddiagnostik in MKG-Chirurgie und Zahnmedizin. Stuttgart-New York: Georg Thieme 2001

Valentin J (2007): The 2007 recommendations of the international commission on radiological protection. Ann ICRP $\underline{37}, 1-332$

Vandenberghe B, Jacobs R, Yang J (2007): Diagnostic validity (or acuity) of 2D CCD versus 3D CBCT-images for assessing periodontal breakdown. Oral Surg Oral Med Oral Pathol Oral Radiol Endod 104, 395-401

Vandenberghe B, Jacobs R, Yang J (2008): detection of periodontal bone loss using digital intraoral and cone beam computed tomography images: an in vitro assessment of bony and/or infrabony defects. Dentomaxillofac Radiol $\underline{37}, 252-260$

Vazquez L, Saulacic N, Belser U, Bernard JP (2007): Efficacy of panoramic radiographs in the preoperative planning of posterior mandibular implants: a prospective clinical study of 1527 consecutively treated patients.

Clin Oral Implants Res $\underline{19}, 81-85$

Viktorov Y: Prävalenz von apikalen Parodontitiden sowie die Häufigkeit und Qualität endodontischer Behandlungen in einer Berliner Population.

Med. Diss. Düsseldorf 2006 
Visser H, Hermann KP, Bredemeier S, Köhler B (2000): Dose measurements comparing conventional and digital panoramic radiography.

Mund Kiefer Gesichtschir 4, 213-216

Voßhans J (2005): Genaue Lagebestimmung der unteren Achter präoperationem. ZM 2 , 32-36

Vrtiska TJ, Hartmann PP, Kofler JM, Bruesewitz MR, King BF, McCollough CH (2009): Spatial resolution and radiation dose of a 64-MDCT scanner compared with published CT urography protocols. Am J Roentgenol 192, 941-948

Webber RL, Messura JK (1999): An in vivo comparison of digital information obtained from tuned-aperture computed tomography and conventional dental radiographic imaging modalities.

Oral Sur Oral Med Oral Pathol Oral Radiol Endod $\underline{88}, 239-247$

Wenzel A (1995): Current trends in radiographic caries imaging. Oral Surg Oral Med Oral Pathol Oral Radiol Endod 으, 527-539

Youssefzadeh S, Gahleitner A, Dorffner R, Bernhart T, Kainberger FM (1999): Dental vertical root fractures: value of CT in detection. Radiology 210, 545-549

Zembic A, Sailer I, Jung RE, Hämmerle CH (2009): Randomized-controlled clinical trial of customized zirconia and titanium implant abutments for single-tooth implants in canine and posterior regions: 3-year results. Clin Oral Implants Res $\underline{20}$, 802-808

Zhang Y, Zhang L, Zhu XR, Lee AK, Chambers M, Dong L (2007): Reducing metal artifacts in cone-beam $\mathrm{CT}$ images by preprocessing projection data.

Int J Radiat Oncol Biol Phys 무, 924-932

Ziegler CM, Woertche R, Brief J, Hassfeld S (2002): Clinical indications for digital volume tomography in oral and maxillofacial surgery.

Dentomaxillofac Radiol $\underline{31}$, 126-130 


\section{Danksagung}

Mein besonderer Dank gilt Frau PD Dr. med. dent. S. Sennhenn-Kirchner für die Überlassung des Dissertationsthemas und die wissenschaftliche Betreuung der Arbeit. Vor allem möchte ich Ihr für die stetige Hilfe und Diskussionsbereitschaft sowohl bei der Durchführung der Versuche als auch bei der schriftlichen Abfassung der Dissertation danken. Herzlichen Dank für die vertrauensvolle Zuwendung.

Besonders danken möchte ich zudem Herrn Dipl. Phys. C. Dullin für die unermüdliche Hilfsbereitschaft bei der Durchführung der fpVCT-Aufnahmen und deren Bilddarstellung, für die vielen Ratschläge zur Gestaltung der Arbeit und das Durcharbeiten des Textes, vor allem aber die Antworten auf die vielen Fragen der technischen Gegebenheiten. Vielen Dank für die guten Zuworte, die stetig durch Dich geförderte Motivation und die fröhliche Zusammenarbeit.

Ebenso möchte ich Prof. Dr. med. dent. C. Hannig meinen Dank ausdrücken; nicht nur für die Idee der Arbeit sondern vor allem für die trotz großer Entfernung andauernde Hilfsbereitschaft bei der Erstellung der Arbeit, die vielen Hilfestellungen und die Diskussionsbereitschaft, die schnellen und präzisen Korrekturarbeiten bei der kritischen Durchsicht des Manuskripts und die unglaubliche Förderung meiner Motivation an der Arbeit.

Mein Dank geht auch an Herrn Dr. med. dent. B. Kirchner, der mir bei der Erhebung der Daten behilflich war und mir im Umgang mit Zahnfilm, Orthopantomograph und dem Digitalen Volumentomographen mit Rat und Tat zur Seite stand. Ebenso möchte ich mich für sein bastlerisches Engagement zur Ermöglichung der Versuche bedanken. Herzlichen Dank für die hervorragende Zusammenarbeit.

Auch danke ich Herrn Dr. med. dent. S. Klotz und Herrn Dr. med. M. Püsken für die tatkräftige Unterstützung bei der Beurteilung der Bilddaten.

Weiterhin danke ich Herrn Dr. rer. nat. K. Jung für die Unterstützung bei den Berechnungen und der statistischen Darstellung. 
Von Herzen danke ich meinem Mann für die vielen Hilfestellungen bei den computertechnischen Fragen und bei den Korrekturarbeiten und die unermüdliche Geduld.

Mein ausdrücklicher Dank gilt auch Frauke Schapp und Nadine Meier für die unermüdliche Unterstützung in allen Fragen und die Förderung meiner Motivation während der gesamten Zeit. 


\section{Lebenslauf}

Lena Jarnot, geb. Nielebock

Ich wurde am 12.06.1980 in Göttingen als Tochter von Regina Nielebock, geb. Böning, und Heinz-Jürgen Nielebock geboren. Ich besuchte von 1986 bis 1990 die Grundschule in Hardegsen und anschließend von 1990 bis 1992 die Orientierungsschule in Moringen. In der Zeit von 1992 bis 1999 besuchte ich weiter das Felix-Klein-Gymnasium in Göttingen, wo ich im Sommer 1999 die allgemeine Hochschulreife erlangte.

Nach Beendigung meiner Aushilfstätigkeit bei „Prolight Lichttechnik GmbH“ in Hardegsen von 1996 bis 2000 absolvierte ich von 2000 bis 2002 die Ausbildung zur Rettungsassistentin an der Hildegard-Vötterle-Schule in Kassel. Anschließend begann ich im Oktober 2002 das Studium der Humanmedizin an der Georg-AugustUniversität in Göttingen. Nach Studienortwechsel zum Beginn des Praktischen Jahres im August 2007 an die Philipps-Universität in Marburg verbrachte ich einen vier-monatigen Auslandsaufenthalt in Kanada/Toronto zur Absolvierung des chirurgischen Teils des Praktischen Jahres. Im Mai 2009 erlangte ich an der PhilippsUniversität in Marburg die Approbation. Während der Studienzeit (2002 bis 2009) ging ich regelmäßig einer Aushilfstätigkeit als Rettungsassistentin beim Deutschen Roten Kreuz in Kassel nach. Zudem war ich freie Mitarbeiterin des CRediT-Registers (2006) und der Flec-SL-Studie B11 des Kompetenznetzes Vorhofflimmern (AFNET) (seit 2007). Im Jahr 2008 führte ich zudem eine Aushilfstätigkeit in einer Psychotherapeutenpraxis für Kinder und Jugendliche in Northeim durch. Meine Tätigkeit als Ärztin begann ich am 1. September 2009 im Klinikum in Kassel als Assistenzärztin der Anästhesiologie und Intensivmedizin, wo ich seitdem tätig bin. 